\title{
A Conceptual Design of the Fast-Liner Reactor (FLR) for Fusion Power
}

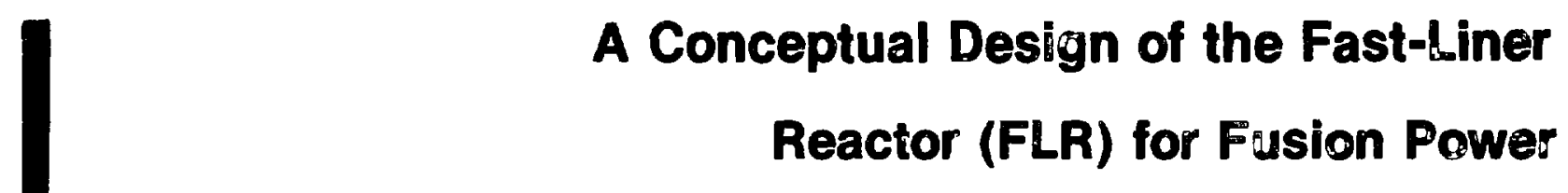


LA.7686-MS

Informal Report

UC-20d

Issued: February 1979

\title{
A Conceptual Design of the Fast-Liner Reactor (FLR) for Fusion Power
}

\author{
R. W. Moses \\ R. A. Krakowski \\ R. L. Miller
}

\section{MAJOR CONTRIBUTORS}

G. E. Cort (heat transfer), R. A. Gerwin (physics), R. L. Hagenson (plasma engineering, costing), J. D. Jacobson (blast confinement), T. R. Jarboe (physics), R. C. Malone (physics), T. A. Oliphant (numerical techniques), P. D. Soran (neutronics), and C. E. Swannack (energy storage and transfer).

This work was supprrted by the US Department of Energy, Office of Fusion Energy, and is part by the Electric Power Research Institute. 
CONTENTS

$\begin{array}{ll}\text { ABSTRACT } & 1\end{array}$

$\begin{array}{ll}\text { I. INTRODUCTION AND SUMMARY } & 1\end{array}$

II. SUMMARY DESCRIPTION OF REACTOR OPERATION 3

III. PHYSICS AND TECHNOLOGY DESIGN BASES

A. Reactor Physics $\quad 7$

1. Plasma Model 7

a. Radial Transport 9

b. Axial Transport 10

c. Burn Dynamics 11

2. Liner Model 11

a. Stability 12

b. Dynamics 13

3. Numerical Methods 14

4. Development of Physics Operating Point 14

B. Reactor Engineering/Technology 20

1. Energy Balance 21

2. Plasma Preparation 23

3. Energy Storage, Switching, and Transfer 24

4. Liner Leads 26

5. Neutronics Analysis 32

6. Blast Containment 39

7. Heat Transfer 46

C. Costing Model $\quad 50$

D. Design Point 52

IV. PRESENT KNOWI.EDGE IN PHYSICS AND TECHNOL.OGY

$\begin{array}{ll}\text { A. Physics Confidence } & 67\end{array}$

1. Plasma Preparation 67

2. Transport 67

3. Liner 68

B. Techno logy 68

1. Liner/Leads Fabrication 68

2. Plasma Preparation 70

3. Containment 70

4. Energy Transfer and Storage 71

5. Leads/Liner Replacement 71

6. Primary Heat-Transfer System 72

$\begin{array}{ll}\text { V. SUMMARY CONCLUSIONS } & 73\end{array}$

$\begin{array}{ll}\text { ACKNOWLEDGMENTS } & 74\end{array}$

APPENDIKES (Including Tatles and Figures) 75

A. Description of LNRBRN Code 75

B. Optimization of Coaxial Leads Structure 78

C. Optimization of Interleaved Leads Structure 88 
D. Description MCNP Monte Carlo Calculation

E. Use of the Virial Theorem and a Simple Shock Model to Estimate Blast Effects in Vacuum and Two-Phase Media

F. Costing Guidelines, Accounting System, and Data Base 


\section{A CONCEPTUAL DESIGN OF THE FAST-LINER}

REACTOR (FLR) FOR FUSION POWER

by

R. W. Moses, R. A. Krakowski, and R. L. Miller

\section{ABSTRACT}

The generation of fusion power from the Fast-Liner Reactor (FLR) concept envisages the implosion of a thin (3$\mathrm{mm}$ ) metallic cylinder (0.2-m radius by $0.2-\mathrm{m}$ length) onto a preinjected plasma. This plasma would be heated to thermonuclear temperatures by adiabatic compression, pressure confinement would be provided by the liner inertia, and thermal insulation of the wall-confined plasma would be established by an embedded azimuthal magnetic field. A 2-to $3-\mu \mathrm{s}$ burn would follow the $\sim 10^{4} \mathrm{~m} / \mathrm{s}$ radial implosion and would result in a thermonuclear yield equal to 10-15 times the energy initially invested into the liner kinetic energy. For implosions occurring once every $10 \mathrm{~s}$ a gross therma? power of $430 \mathrm{MWt}$ would be generated. The results of a comprehensive systems study of both physics and technology (economics) optima are presented. Despite unresolved problems associated with both the physics and technology of the FLR, a conceptual power plant design is presented.

\section{INTRODUCTION AND SUMMARY}

The use of magnetically driven, metallic liners for the adiabatic compression of D-T plasmas to thermonuclear conditions has been studied by a number of investigators, $1-4$ The largest imploding-liner programs to date have been at the Kurchatov Institute in the USSR ${ }^{2}$ and at the Naval Pesearch Laboratory (NRL) in the United States. ${ }^{3}$ The approach taken by the Kurchatov group has emphasized fast $\left(10^{3}-10^{4} \mathrm{~m} / \mathrm{s}\right)$ implosions of thin metal shells in a varieity of configurations, whereas the NRL group has been concerned primarily with slower $\left(\sim 10^{2} \mathrm{~m} / \mathrm{s}\right)$ implosions of more massive, cylindrical systems. The 
Los Alamos Scientific Laboratory has proposed ${ }^{5}$ and is conducting experiments on $\sim 10^{4} \mathrm{~m} / \mathrm{s}$ imploding liners; this approach is similar to that followed ten years ago by Alikhanov et al. ${ }^{6}$ Consideration of liner buckling and Rayleigh-Taylor stability, ${ }^{7}$ particle and energy confinement, and the desire for very compact systems exhibiting high power densities nave led to the choice of the fast mode. Fast implosions that are driven by an azimuthal field should alleviate the Rayleigh-Taylor instability and supress the plastic-elastic (buckling) instability ${ }^{8}$ in addition to allowing wallconfinement of the plasma pressure. The technological problems associated with GJ-level energy transfers and releases over microsecond time intervals are severe, ${ }^{9}$ and to a great extent the magnitude of these problems is related directly to the non-ideal behavior of a fast-liner/plasma system (i.e., liner compressibility, liner stability, field diffusion, plasma turbulence, thermal conduction, arid radiation) as reflected by constraints imposed by a realistic engineering energy balance.

The Fast-Liner Reactor (FLR) concept combines the favorable aspects of inertial confinement and heating with the more efficient energy transfer associated with magnetic approaches to yield a conceptual fusion system based on the pulsed burn of a very dense D-T plasma. A thin metal cylinder or "liner" of $\sim 0.2-\mathrm{m}$ initial radius, $\sim 3-\mathrm{rm}$ initial thickness, and $\sim 0.2-\mathrm{m}$ length is imploded radially to a velocity of $\sim 10^{4} \mathrm{~m} / \mathrm{s}$ by self-magnetic fields resulting from large currents driven axially through the liner. The liner implodes onto a $\sim 0.5-\mathrm{keV}, \sim 10^{24}-\mathrm{m}^{-3} \mathrm{D}-\mathrm{T}$ plasma that is initially formed in or injected into the liner. As the liner implodes in $\sim 20-40 \mu \mathrm{s}$, adiabatic compression raises the plasma to thermonuclear temperatures, and a vigorous fusion burn ensues for $\sim 2-3 \mu \mathrm{s}$. During the implosion the plasma pressure is confined inertially by the metal liner and endplug walls. An imbedded azimuthal magnetic field, generated by an axial current driven through the plasma, provides magnetic insulation against radial and axial thermal conduction losses. The energy released by each implosion is sufficient to destroy the liner assembly and a few meters of adjacent electrical leads. Between implosions $(\sim 10-20$ s) the previously destroyed liner and leads are replaced by a fresh assembly. The FLR would require a relatively small ( 22.5-to 3.0-m radius) containment vessel and wouid operate with high engineering power density $\left(\sim 5-10 \mathrm{MWt} / \mathrm{m}^{3}\right)$. The recirculating 
power fraction is anticipated to be in the range $0.15-0.30$.

On the basis of detailed physics modeling an FLR operating puint is reported, and a conceptual reactor embodiment is described. The major engineering and technology problems associated with the FLR concept, in orjer of perceived importance are a) the economics of recycling routinely destroyed ieads and liners, b) the means of plasma preparation, c) the containment of repeated blasts, d) the switching and transfer of large quantities of fast-pulsed energy (1-2 GJ, 20-30 $\mu \mathrm{s})$ to the 1:iner, e) the means by which liners and leads are replaced every 10-20 s. Although the limited scope of this study does not allow a comprehensive or self-consistent analysis of each of these problem areas, an assessment of both physics and technology is presented, and possible solutions to each problem area are proposed.

Section II gives a summary description of the FLR operation and the physics operating point selected on the basis of a cost analysis. Although the physics operating point represents an optimum, insofar as the liner dynamics and achievable tecinnology is concerned, no attempt was made to optimize fully on the basis of cost. Comprehensive descriptions of the physics, engineering/technology, and costing bases are found in Sec. III, which concludes with a detailed description of the reactor point design (Sec. III.D). Since many of the analytic tools required to arrive at the FLR design point had to be "invented" and/or developed specifically for this study, the evolution and implementation of these design tools are discussed in detail in appropriate appendixes. Section IV concludes this report with an assessment of present knowledge associated with both physics and technology issues for the FLR approach.

\section{SUMMARY DESCRIPTION OF REACTOR OPERATION}

The computational base used to arrive ait the FLR design point is described in Secs. III.A-B. Trade-off studies (Sec. III.A.4) have identified two neariy optimum physics design points, which are summarized in Table II-I. First a "low-yield" case relaxes ine requirements anticipated for the energy transfer and storage: (ETS) system and blast confinement: this low-yield case is marginally acceptable from the viewpcint of recirculating power and economics; the "high-yield" case reverses this emphasis.

On the bissis of the physics ani energy-balance design point selected for the low-yield case in Table II-I, a number of blast-containment schemes $9,10,11$ 
TABLE II-I

INTERIM FLR PHYSICS OPERATING POINTS

Design Parameter, symbol (units)

Initial liner inner radius, $r_{10}(\mathrm{~m})$

Initial liner thickness, $\Delta_{0}(\mathrm{~mm})$

Initial azimuthal field, $B_{10}(T)$

Initial liner energy, $W_{L}(G J)$

Liner Q-value, Q

Pure fusion yield, $Q W_{L}$ (GJ)

Enhanced fusion yiald $\left(M W_{N}+W_{\alpha}\right)$ (GJ)

Engineering Q-value, $Q_{E}^{(a)}$

Recirculating po er fraction, $\varepsilon=1 / Q_{E}$

Cycle time, $\tau_{c}(s)(b)$

Total thermal power, $\mathrm{P}_{\mathrm{TH}}$ (Mit)

Gross electric power, $\mathrm{P}_{E T}$ (MWe)

Recirculating power, $\mathrm{P}_{\mathrm{C}}$ (MWe)

Net electric power, $P_{E}$ (MWe)

Thermal power density, $\left(\mathrm{MWt} / \mathrm{m}^{3}\right)(\mathrm{c})$

Number of units for 1000 Mule (net)

Revenue per shot at $40 \mathrm{mills} / \mathrm{kWeh}$ (\$)

Net plant efficiency, $n_{p}=n_{T H}(1-\varepsilon)$
Low Yield

High Yield

$\begin{array}{rr}0.2 & 0.3 \\ 3.0 & 4.5 \\ 13.0 & 13.0\end{array}$

$0.336 \quad 0.756$

10.7

14.7

3.56

11.11

3.92

12.22

3.94

5.28

0.25

0.19

10.0

10.0

430.

1300.

172.

520.

43.

99.

129.

5.8

421.

7.8

19.9

2.3

14.27

46.79

0.30

0.32

(a) AT1 quantities needed to determine $Q_{E}$ have been specified in the text, except for $n \mathrm{YNT}^{\mathrm{NT}}$. On the basis of a preliminary economic optimization of the leads structure (Sec. III.B.4) $n$ fNi $=0.9$.

(b) Chosen on the basis of an estimate of the time needed to replace leads and liner.

(c) The system power density is based on the total volume enclosed by a $2.6-\mathrm{m}-$ radius containment vessel of wall thickness $0.3 \mathrm{~m}$. The size of the blast radius is based on structural calculations given in Sec. III.B.6. 


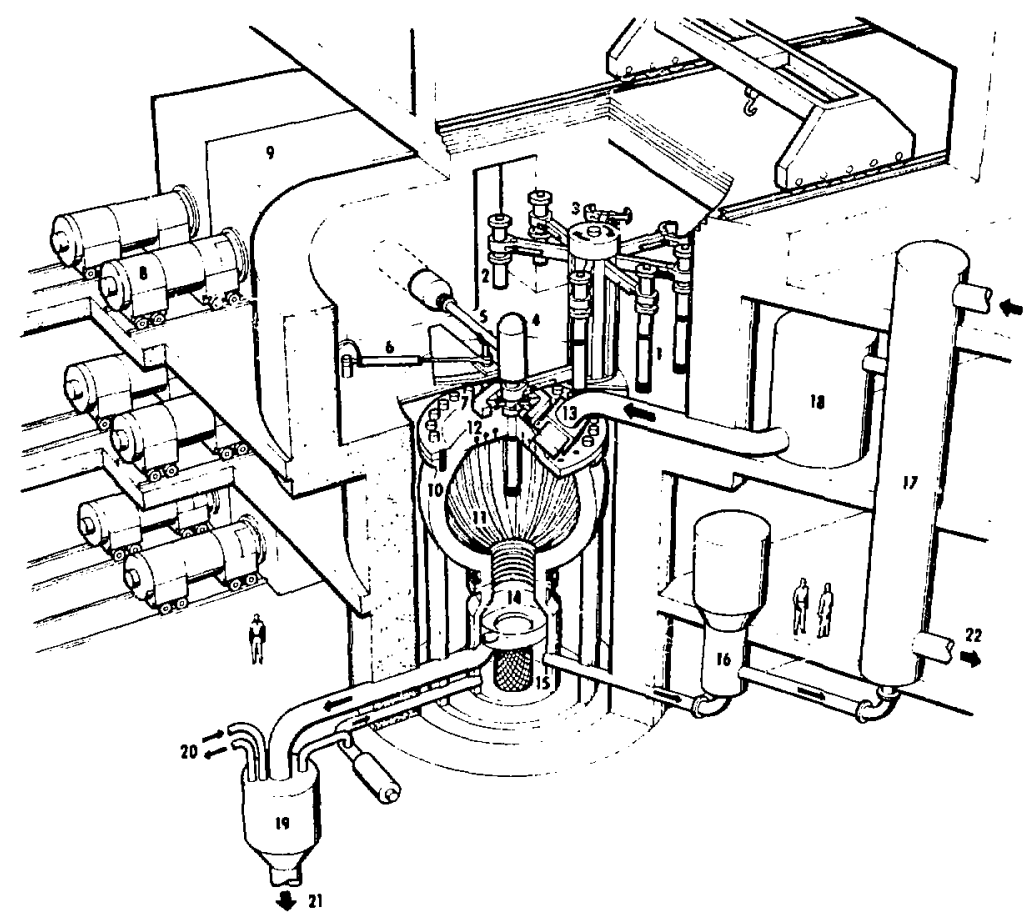

Fig. II-1. Isometric drawing of Fast-liner Reactor nuclear island for the low-yield case given on Table II.I. Component identification: (1) liner/leads assembly ready for implosion; (2) remains of imploded-liner/leads assembly; (3) liner/leads carouse 1; (4) plasma preparation; (5) power leads; (6) hydraulic arm to move power connection; (7) blast vesse 1 head and liner/leads feedthrough; (8) homopolar motor/generator; (S) inductive transfer element, transfer capacitor, and switches; (10) blast vessel (2.6-m radius, 013-m wall thickness); (11) shock extending ribs; (12) lithium-spray spargers; (13) lithium inlet and control valve; (14) solid debris skimmer; (15) lithium sump and storage; (16) lithium pump; (17) Li/Na heat exchanger; (18) lithium surge and storage tank; (19) solid debris separation; (20) lithium drag stream to tritium recovery; (21) solids debris to recovery and refabrication; (22) secondary sodium coolant.

were conceived and are described in Sec. III.B.6 and Sec. IV.B.3. A lithium (or lithium-lead) spray was adopted by this study as a coolant/blast-mitigating/breeder medium and used to project the FLR embodiment further. The essential operating components of this approach are shown in Fig. II-1 and are described below. A conceptual 1000-MWe (net) power plant that is based on this concept is described in jec. III.D.

The liquid-metal spray concept is similar to a scheme proposed by Burke et al.12 for an electron-beam pellet fusion scheme. Referring to Fig. II-1, fresh liner/leads assemblies [1] coming from a refabrication facility (not shown) are transported to the FLR core as spent liner/leads assemblies [2], and 
are removed for reprocessing by the rotating liner manipulator [3]. A liner/leads assembly is inserted through a port in the blast-containment header, and the plasma source and the connector module for the energy transfer and storage (ETS) system [4], which is attached to the external ETS leads arm [5], is moved into place [6]. The liner/leads assembly is clamped to the containment vessel by a latching assembly [7]. The $\sim 450-M J$ power supply consists of a bank of homopolar generators [8], an intermediate storage inductor, intermediate transfer capacitors and switches [9], all of which are shown approximately to scale. The neariy spherical FLR pressure vessel [10] with the shaped inner surface, incorporating shock suppression ribs [11] is riuminally 0.3-m-thick stainless steel designed to contain repetitive explosive releases of $\sim 1-2 \mathrm{GJ}$. Blast mitigation, tritium breeding, and heat transfer to the external thermal cycle are provided by a molten Li (or LiPb) spray or "rain" that is injected from the upper inlet manifold [12] through the reactor cavity as the liner implodes. Flow control is provided by the isolation valve [13]. During and after each liner shot a mixture of heated Li coolant and liner/leads debris falls to the debris trap [14] and thermal storage sump [15] below. The mixed-mean temperature rise in the $\approx 50$ v01\% iithium spray contained within the blast vessel amounts to $\sim 60 \mathrm{~K}$, the temperature difference ultimately appearing across the primary Li/Na heat exchanger [17]. The primary coolant pump [16] continuously draws off the Li coolant for circulation through the primary heat exchanger [17], surge tank [18], and back to the blast cavity. The debris removal system [19] returns insulator and liner/leads material to the refabrication facility [21] for reconstitution into new assemblies. The leads structure is composed of solid $\mathrm{Li}$ or $\mathrm{LiPb}$ conductor and a glass-like insulator; the conductor material is recovered and extruded into a new leads assembly, but the glass-like electrical insulator is discarded as slag. A tritium recovery system [20] draws off a fraction of the circulating Li coolant. An intermediate coolant loop [22] isolates the nuclear island from the turhogenerator (not shown). For economic reasons an FLR plant may consist of several reactor cavities operating sequentially and sharing a common ETS system and balance of plant. Approximately eight of the 130-MWe(net) units depicted in Fig. II-1 would be required to deliver 1000 MWe(net); this modular approach has been adopted by the costing analysis and is discussed further in Sec. III.D. 


\section{I. PHYSICS AND TECHNOLOGY DESIGN BASES}

This section quantitatively describes the computational basis for both the burn physics and the engineering design. Because of the unique approach of the inertially confined, magnetically insulated FLR, many of the computational tools had to be developed specifically for this study. Although these models represent the state of the art for this concept, these approximate models are nevertheless preliminary, have yet to be tested against experiment, and remain in a developmental stage.

A. Reactor Physics

Figure III-1 depicts a cylindrical liner configuration as it implodes onto a preinjected plasma in which is embedded an insulating azimuthal magnetic field $B_{\odot}$, whereas Fig. III-2 depicts a more schematic view. Typical dimensions for an unimploded liner would be $0.2-\mathrm{m}$ radius and $0.2-\mathrm{m}$ length. The field $B_{\Theta}$ is created by an axial plasma current $I_{p}$ as the liner is imploded with a radial velocity $v_{1}$ by an external azimuthal field caused by an axial drive current $I_{d}$ (Fig. III-1). A radial, time-dependent computer code LNRBRN has been developed to model both the plasma burn and liner implosion dynamics. Both the physics basis and the numerical procedures embodied in the LNRBRN code are described in this section; a description of the LNRBRN code is found in Appendix A.

1. Plasma Mode1. The plasma is treated as a single-fluid gas in cylindrical geometry with an embedded magnetic field $B_{\ominus}$; a radially uniform axial current is assumed to establish this embedded field. The LNRBRN model computes radial thermal conduction and field diffusion in the MHS approximation* while incorporating an analytic approximation for axial thermal conduction as a function of radius. Bremsstrahlung and D-T burnup are computed at the plasma midplane as functions of radius. Alpha-particle reating of the plasma is not considered, since the alpha-particie mean-freepath for thermalization is several times the plasma radius at peak compression. The plasma and field pressures are computed at the plasma-boundary and are dynamically coupled to the plasma-liner motion.

*The magnetohydrostatic (MHS) model treats all but the inertial termis in the MHD approximation. 


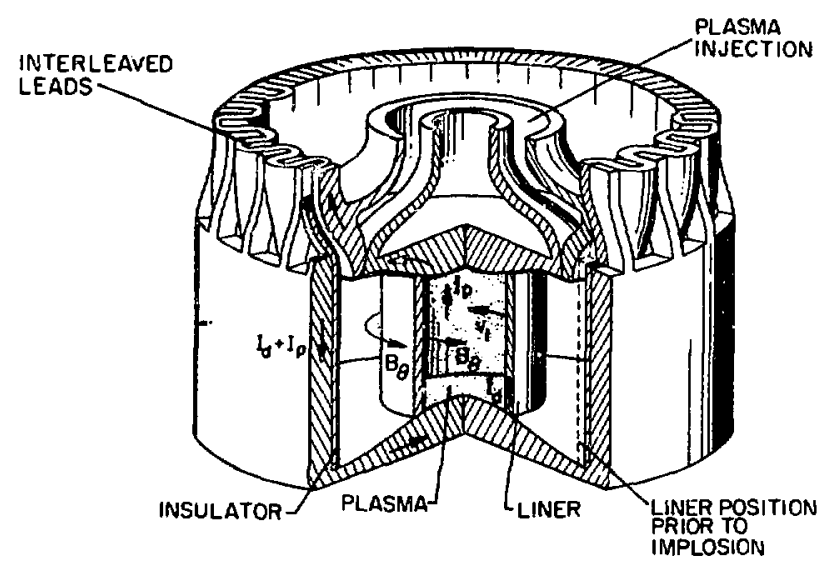

Fig. III-1. Schematic diagram of 0.2-m initial radius and $0.2-\mathrm{m}-1$ long 1 iner assembly showing (a) plasma current $I$ that generates internal azimuthal field $B_{A}$ for thermal insulation between plasma (inside inner vessel) and liner wall, (b) Tiner drive current I that causes the external azimuthal field $B_{\text {f }}$ to drive the liner inward with a velocity $v_{1}$. A "force-reduced" interleaved Peads structure and a port for coaxial plasma injection are shown.

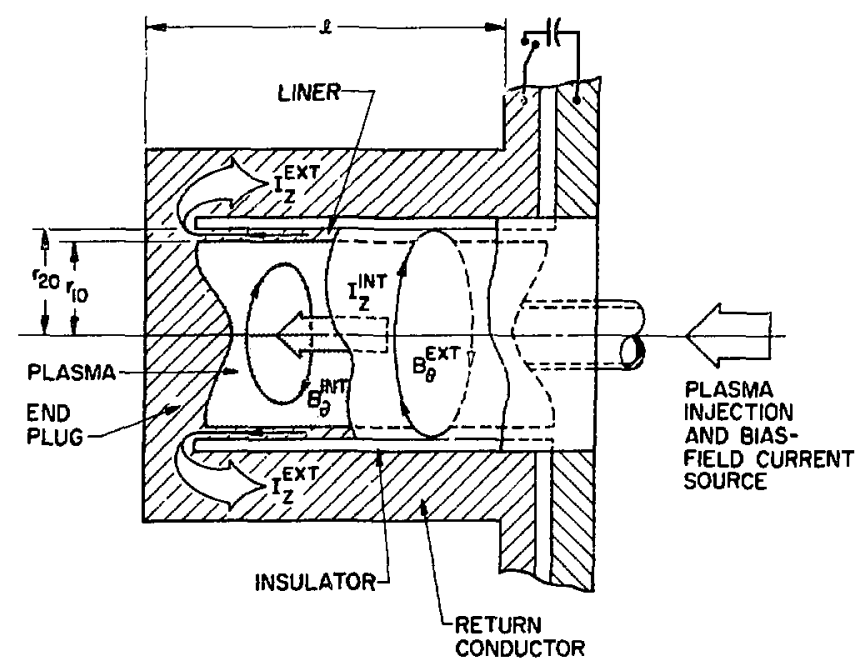

Fig. III-2. Schematic diagram of 0.2-m initial radius and 0.2-m-long liner assembly showing in more detail the 1 iner per se, the internal axial current I INT creating the insulating field $\mathrm{B}_{\Theta}$, and the drive current IEXT creating the drive field $B E X T$. Massive return conductors, the electrical insulation, and feedplate leads structure are shown. 
a. Radial Transport. LNRBRN is an implicit Lagrangian code. Sound transit times in a typical liner plasma are much less than the impiosion time; inertial terms, therefore, can be neglected and plasma motion is determined by pressure balance $(\vec{J} \times \vec{B}=\vec{\nabla} P)$ for equal electron and ion temperatures. This MHS pressure balance can be transformed to the following integral equation when the magnetic field exhibits only the azimuthal or " $\Theta$ " direction

$$
2 n k_{B} T+B_{\theta}^{2} / 2 \mu_{0}=\left(4 / r^{2}\right) \int_{0}{ }^{r} n k_{B} T r^{\prime} d r^{\prime},
$$

where $k_{B}$ is the Boltzmann constant $\left(1.6(10)^{-16} \mathrm{~J} / \mathrm{keV}\right), n\left(1 / \mathrm{m}^{3}\right)$ is the ion density $\mu_{0}=4 \pi(10)^{-7} \mathrm{H} / \mathrm{m}$, and $T$ is expressed in keV units.

Plasma parameters are computed as functions of time by a two-step method. ${ }^{13}$ First, the Lagrangian mesh is fixed in space, ind all diffusion and loss processes are evaluated for a given time step. The basic equations are

$$
\begin{aligned}
& 2 n k_{B}(\partial T / \partial t)=(1 / r) \partial[r k \partial T / \partial r] / \partial r+S, \\
& \mu_{0}\left(\partial B_{\theta} / \partial t\right)=\partial\left[n\left(B_{\theta} / r+\partial B_{\theta} / \partial r\right)\right] / \partial r, \text { and } \\
& \partial n / \partial t=-n^{2}<\sigma v>/ 2,
\end{aligned}
$$

where $k$ is the thermal conductivity, ${ }^{14} n$ is the electrical resistivity, ${ }^{14}$ $S$ is a net volumetric power source (or sink), and $\langle\sigma v\rangle$ is the Maxwellianaveraged D-T fusion reactivity. Since the alpha particles are assumed to escape unthermalized from the plasma, charge neutrality requires that two electrons also escape the plasma for each fusion reaction.

The bremsstrahlung power density, as used in the source term $S$, is taken as 15

$$
S_{B R}\left(W / m^{3}\right)=-5.35(10)^{-37} n^{2} T^{1 / 2} \tanh \left(T / T_{W}-1\right),
$$

where $T_{W}$ is an assumed wall temperature. The hyperbolic tangent has been incorporated into the usual bremsstrahlung expression in order to approximate radiation reabsorption by the dense plasma immediately adjacent to the wall. The plasma performance, as predicted by LNRBRN, is generally insensitive to 
the assumed value for $T_{W}$. After Eqs. (III-2)-(II $\left.i-4\right)$ are solved for a given time step, the Lagrangian mesh is adjusted in space to reestablish pressure balance (Eq. (III-I)) and these equations are then coupled dynamically to the liner behavior (Sec. III.A.3 and Appendix A). This procedure completes the above-mentioned two-step approach.

b. Axial Transport. An analytic model for axial thermal conduction was derived $^{16}$ to give an axial conduction heat-sink term, $\mathrm{S}_{\mathrm{CZ}}$, for use in Eq. (III-2). The results from Eqs. (III-2)-(III-4) are representative of the midplane in a liner plasma of length $\ell$. Piasma parameters are expected to be nearly constant in the axial direction except near and within the high-density, low-temperature sheath near the endplug (Fig. III-1).

The axial conduction model assumes (a) axial and radial thermal conduction are separable, (b) fields and plasma pressures are independent of axial position, (c) thermal conductivity ${ }^{14}$ can be divided into three regions according to the magnitude of $\omega \tau$ for ions and electrons, where $\omega$ is the gyrofrequency and $\tau$ is the respective collision time.

Region I $\left(\omega_{i} \tau_{i}>1\right)$

$$
k_{i}=8.0(10)^{-39} n^{2} \ln \Lambda / T^{1 / 2} ;
$$

Region II $\left(\omega_{e} e^{\tau} e^{>1, \omega_{i} \tau}{ }_{i}^{<1)}\right.$

$$
k_{i}=2.5(10)^{13} T^{5 / 2} / \ln \Lambda \quad ;
$$

Region III $\left(\omega e^{\tau} e^{<1)}\right.$

$$
k_{e}=1.5(10)^{15} T^{5 / 2} / \ln \Lambda
$$

where except for $T(k e V)$, mks units are consistently used. For $Z=1$ and an average $D-T$ atom $(A=2.5), \omega^{\tau} i^{j}=4.0(10)^{25} B_{\theta} T^{3 / 2 / n \ell n \Lambda}, \quad \omega^{\tau} e=$ $1.9(10)^{27}{ }_{\theta} T^{3 / 2} / n \ell n \Lambda$, and $\Lambda=9.32(10)^{16^{\top}} \mathrm{T} / \mathrm{n}^{1 / 2}$.

Assumption (d) stipulates that Region III is a small and probably turbulent space near the endplug that can be neglected. The transition between Regions $I$ and II is defined by $\omega_{i} \tau_{i}=1$ for each radius; $a$ corresponding transition temperature and axial position, $T_{t}$ and $z_{t}$, can be 
defined. On the basis of the constant-pressure assumption the cross-field ion thermal conductivities are given by

$$
\begin{array}{ll}
k_{i}=C_{I} T^{-5 / 2} & \text { (Region I) } \\
k_{i}=C_{I I} T^{5 / 2} & \text { (Region II), }
\end{array}
$$

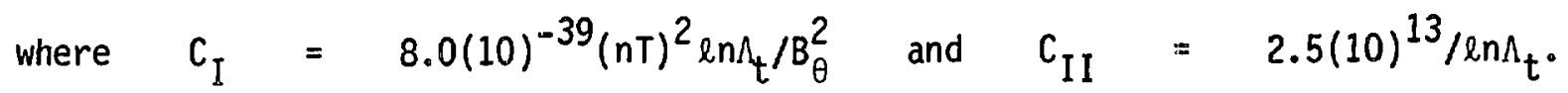
Considering only axial thermal conduction and the constant pressure assumption, the axial heat conduction equation can be integrated from the liner midplane $(z=0, \partial T / \partial z=0)$ to any value of $z$, this result is then integrated over the liner length to give an effective axial conduction power loss per unit volume at a given radius.

$$
S_{C Z}\left(W / m^{3}\right)=-\left(16 / l^{2}\right)\left[C_{I}\left(T-3 / 2-T^{3 / 2}\right) / 3-C_{I I} T_{t}^{7 / 2} / 7\right] \cdot
$$

The source term $S$ in Eq. (III-2) is equal to the sum of $S_{B R}$ (Eq. (III-5)), $\mathrm{S}_{\mathrm{CZ}}$ (Eq. (III-1I)), and joule heating terms (alpha-particle heating is insignificant).

c. Burn Dynamics. The thermonuclear reaction rate $n^{2}<\sigma \vee / 4$ for a $50 / 50$ DT fuel mixture is computed as a function radial position at the $z=0$ midplane using tabular values for the D-T, Maxwellian-averaged reactivity, $\langle\sigma v\rangle$. Since the alpha-particle mean free path classically is expected to excead the (compressed) plasma dimensions, alpha particles are assumed lost and, hence, do not contribute to the plasma energy or pressure. If a significant portion of the alpha-particle energy were to be retained by the plasma, compression would be inhibited, and the fusion yield would be diminished for the optimized physics operating point reported here. Furthermore, the influence on the liner of the thermal flux associated with unthermalized alpha particles is not treated by the LNRBRN model.

2. Liner Model. The pressures and accelerations to which the liner will be subjected are significant, and both compressibility and hydrodynamic stability must be taken into account. Although detailed MHD codes, such as CH.IMISA, ${ }^{17}$ are available with appropriate equation-of-state data and field-diffusion models, such code systems are too cumbersome for use in the present parametric systems analysis. Consequently, LNRBRN uses a simplified 
analytic model of the liner, ${ }^{17}$ and has shown good agreement with the predictions of the detailed CHAMISA code system.

a. Stability. Four potentially disruptive effects on liner motion have been considered: ${ }^{5}$ the Rayleigh-Taylor instability, liner buckling, the sausage instability, and manufacturing asymetry. The Rayleigh-Taylor instability arises when the boundary of two fluids of unequal density or a single fluid with a density gradient is accelerated in the direction of the density gradient. Treating the liner as a fluid, the condition for onset of this instability occurs at the outer surface as the liner is accelerated by the driving magnetic field. A similar instability may develop inside the liner as it is decelerated by the compressed plasma. ${ }^{7}$ The liner physically yields and may be regarded as plastic or liquid shortly after compression by the driving field begins; detailed analysis, 5 however, indicates the growth rate (relative to the implosion time) of Rayleigh-Taylor modes will be substantially reduced by a high viscosity of the liner metal. Experimental evidence $^{18}$ indicates that for aluminum or copper the high pressure of the liner environment increases the viscosity sufficiently to eliminate the need for liner rotation, as is required for the "slow" liner approach."

The buckling instability can occur when an inward force is applied to a stiff convex shell, such as occurs when the drive field acts on the liner. According to preliminary studies 5,8 the azimuthal or "Z-pinch" drive field is sufficiently stabilizing in the azimuthal or " $\theta$ " direction to reduce significantly the potential for liner buckling. Conversely, the Z-pinch field is destabilizing with respect to sausage modes in the axial direction. The latter instability is similar to the Rayieigh-Taylor modes and may be regarded as an additional term in that analysis. Determination of the significance of sausage modes is an objective of the LASL Fast Liner Experiment. ${ }^{5,19}$

Finally, potentially adverse disturbance of liner motion may arise from manufacturing asymmetry. If, for example, the liner has an uneven thickness, the thinner parts will implode faster, causing an irregular liner shape at peak compression. This effect is more severe for high compression ratios. Experimental studies will undoubtediy lead to increased understanding of the required manufacturing tolerances. ${ }^{19}$ It is noted that the fast-implodingliner experiments performed both in the USA ${ }^{20-22}$ and in the USSR ${ }^{2}$ have encountered no significant liner stability problems. 
b. Dynamics. An analytic liner model has been developed on the basis of the impulse-momentum approximation ${ }^{17}$ and is used in LNRBRN. In this inodel, the equation of state for the liner is approximated by

$$
P / B_{0}=\left[(\rho / \rho)^{B^{\prime}}-1\right] / B^{\prime},
$$

where $F$ is pressure, $\rho$ and $\rho_{0}$ are densities with and without pressure, respectively, $B_{0}$ is the bulk modulus at low pressure, and $B^{\prime}$ is a dimensionless parameter which is used to fit Eq. (III-12) to empirical data for a wide range of pressures. 17

The inner and outer radii of the liner are defined as $r_{1}$ and $r_{2}$, respectively, and the comined plasma and field pressure at the inside surface of the liner is defined as $P_{1} \equiv P\left(r_{1}\right)$. The impulse-momentum model gives the pressure within the liner walls as a function of radius

$$
P(r) \simeq P_{1}\left[\frac{r_{2}-r}{r_{2}-r_{1}}\right]^{B^{\prime} /\left(B^{\prime}-1\right)} \text {. }
$$

Likewise, the radial dependence of liner density is given by

$$
\rho ! \rho_{0} \simeq\left[1+\left(B^{\prime} P_{1} / P_{0}\right)\left(\frac{r_{2}-r}{r_{2}-r_{1}}\right)^{1 / B^{\prime}}\right]^{B^{\prime} /\left(B^{\prime}-1\right)} .
$$

The motion for the liner is described by ${ }^{23}$

$$
d^{2}(\bar{R}) / d t^{2}=\left(\tilde{c} \pi / \rho_{0} A_{0}\right)\left[P_{1} r_{1}+\int_{r_{1}}^{r_{2}} P d r\right] \text {, }
$$

where $A_{0}$ equals $\pi\left(r_{20}-r_{10}\right)$, and $\bar{R}$ is a mass-averaged radius given by

$$
\ddot{R}=\left(2 \pi / A_{0}\right) \int_{r_{1}}^{r_{2}}\left(\rho / \rho_{0}\right) r^{2} d r
$$

Equations (III-15) and (III-16) are coupled to the plasma mution and solved numerically, as described in Sec. III.A.3.

The essential approximations regarding liner dynamics are encompassed in 
Eqs. (III-12) and (III-13). The analytic form of the equation of state eliminates the need for a stepwise analysis of the liner over its radial thickness. The impulse-momentum approximation leading to Eqs. (III-13) and (III-14) assumes that relative velocities within the liner are considerabiy less than the initial liner velocity and that the sound transit time in the liner is short compared to characteristic implosion time. Comparisons between this approximate and analytic method with the CHAMISA code have given excelleit agreement. 17

3. Numerical Methods. As noted in Sec. III.A.1.a., LNRBRN is based on a two-step numerical method ${ }^{13}$ in which the transport equations are solved on a fixed Lagrangian spatial mesh; the mesh is subsequently readjusted adiabatically at each time step to satisfy pressure balance (Eq. (III-1)). The plasma is treated as an ideal single-particle gas, and azimuthal flux conservation is imposed. An iterative scheme matches the plasma radius and pressure to the liner radius $r_{1}$ computed with the liner dynamics model, Eqs. (III-15) and (III-16). A description of the LNRBRN numerical procedure and logic fiow is described in Appendix A. A complete time-dependent description of all liner, plasma, and thermonuclear yield parameters is given by LNRBRN. Generally, the most important final result for a given input (i.e., initial plasma density and temperature, initial plasma beta, liner geometry, and input energy) is the ratio of initial iiner energy $W_{L}$ to the sum of fusion neutron yield $W_{N}$ and alpha-particle yield $W_{\alpha}$. This "liner" or scientific $Q$-value, $Q=\left(W_{N}+W_{\alpha}\right) / W_{L}$, is the object function used in all physics optimizations described in the following section; the liner Q-value represents the essential interface between the liner physics and the FLR engineering design. Section III.B.I describes the major system efficiencies that relate $Q$ to the engineering $Q$-value, $Q_{E}$ (recirculating power fraction $\left.\varepsilon=1 / Q_{E}\right)$.

4. Development of Physics Operating Point. To obtain liner Q-values greater than 10 , as required for an economical reactor (Sec. III.B.1), an analytic plasma-liner model was used to estimate a starting point for the optimization of $Q$; this lossless model indicates liner energies on the order of $W_{L}=1-2 \mathrm{GJ} / \mathrm{m}$ and initial plasma line densities of $1-2(10)^{23} \mathrm{~m}^{-1}$. Initial liner parameters, that are compatible witr these criteria in a copper liner, are inner radius $r_{10}=0.2 \mathrm{~m}$, thickness $\Delta_{0}=3 \mathrm{~mm}$, velocity $v_{10}=$ $10^{4} \mathrm{~m} / \mathrm{s}$, and plasma density $n_{0}=10^{24} \mathrm{~m}^{-3}$. Other initial conditions 
include a plasma temperature $T_{0}$ and an initial insulating magnetic field at the plisma/liner interface $B_{10}$. The analytic model of a lossless plasma ${ }^{17}$ precicts $T_{0} \simeq 0.5 \mathrm{keV}$ for initial liner conditions specified above. The initial azimuthal magnetic field is assumed to arise from a uniform axial current $I_{p}$ in the plasma (Fig. III-1).

Since pressure balance (Ea. (III-I)) is always satisfied, the temperature and density cannot be uniform. Hence, $T_{0}$ and $n_{0}$ are initially specified on the axis; the bulk of the plasma is fixed at $T_{0}$, but near the wall the temperature drops smoothly to $T_{W}$. With temperature, field, and density specified, Eq. (III-1) is solved for $n(r, t=0)$. Equations (III-2)-(III-4) are solved, with the initial profiles so determined, and always maintaining local pressure balance (Eq. III-1). The pressure exerted by the plasma and field on the imploding vall is used as one component in the solution of the liner equations of motion. Figure III-3 gives an example of the time dependence of the inner radius $r_{1}$, fusion power $P_{f}$, and total liner losses $W$; ohmic heating of the liner is not included in $W$. The liner dynamics include an analytic approximation to the liner compressibility, this compressibility model being verified by detailed hydrodyramic computations (CHAMISA). ${ }^{17}$

Having consistently specified the initial liner dimensions and velocity, a three-parameter search in initial density, temperature, and insulating field was made to determine the optimum liner Q-value. Rather than specifying

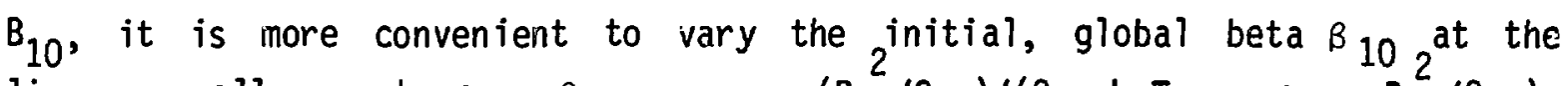
liner wall, where $B_{10}=\left(B_{10}^{2} / 2 \mu_{0}\right) /\left(2 n_{10} k_{B} T_{W}+B_{10}^{2} / 2 \mu_{0}\right)$. Figures III-4 and III-5 show the dependence of $Q$ on $T_{0}$ and $n_{0}$ for two initial liner energies; the initial liner and plasma parameters were adjusted by analytic scaling relationships to preserve $Q$ near optimum. Shown also on Figs. III-4 and III-5 is the relationship between $B_{10}$ and $B_{10}$, as determined by pressure balance (Eq. (III-1)). For each $\beta_{10}$ a maximum $Q$ in $\left(T_{0}, n_{0}\right)$ space is found.

The maxima shown in Figs. III-4 and III-5 result from a complex interaction between the liner and plasma. The liner Q-value decreases with decreasing $T_{0}$ because adiabatic compression to thermonuclear temperatures becomes less efficient, resulting in a higher compression and shorter "dwell" or burn time. Furthermore, once the overcompressed state is reached, the higher plasma density at this point results in an increased radiation loss. On the other hand, for high values of $T_{0}$ the peak temperacure is reached at 


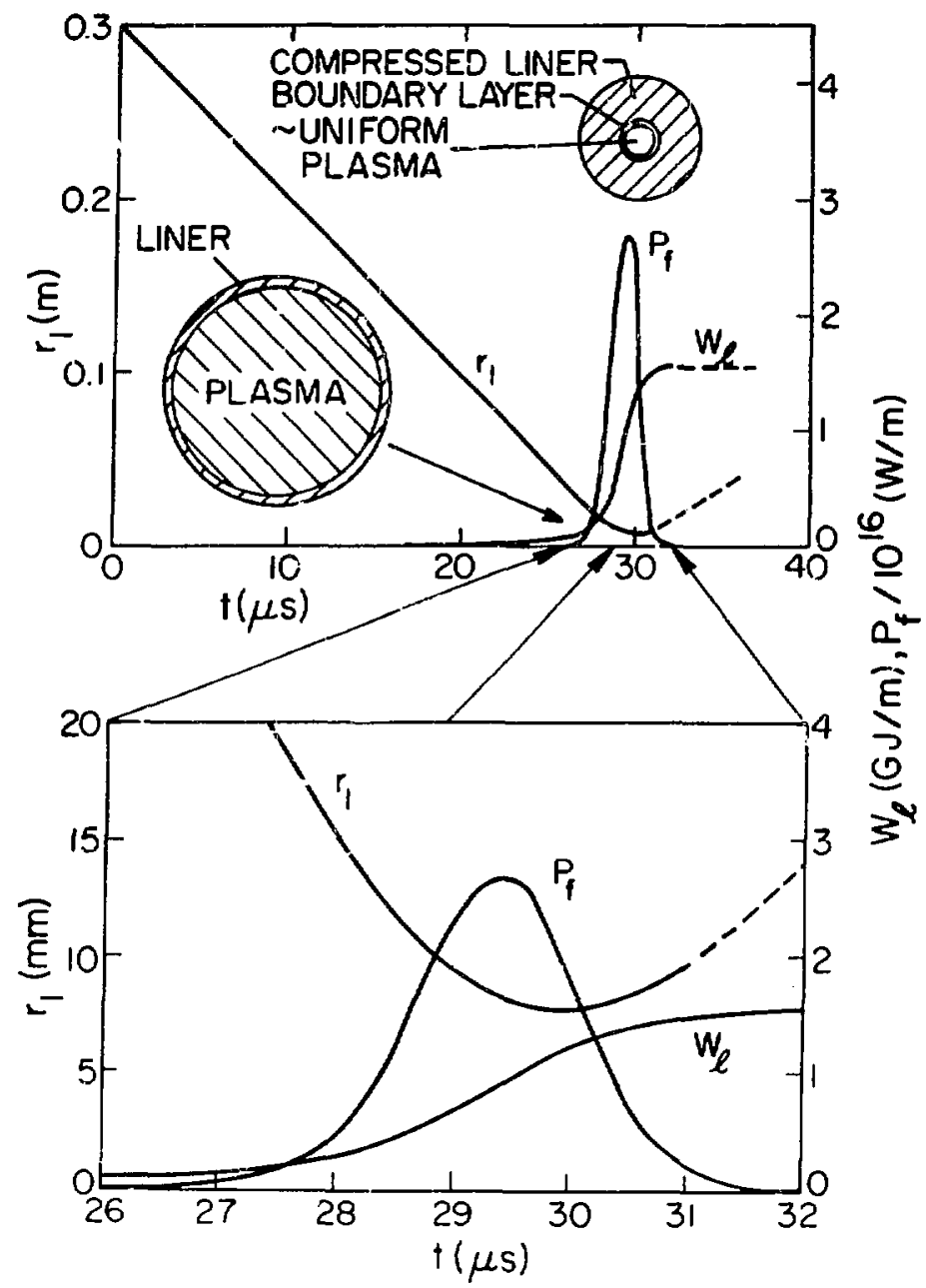

Fig. III-3. Typical response of liner and plasma as predicted by the MHS burn code LNRBRN. Shown is the time dependence of the inner liner radius $r_{1}$, the fusion power per unit length $\mathrm{F}_{\mathrm{f}}$, and the total energy lost during the compression $W_{2}$.

lower compressions and densities, and the corresponding peak reaction rate $\left(n^{2}<\sigma v>\right)$ decreases raster than the dwe 11 time increases. Since more insulating magnetic field exists at the wall for low values of $\beta_{10}$, the increase in $Q$ with decreased $\beta_{10}$ reflects better magnetic insulation.

Similar processes give rise to a maximum $Q$ at fixed $T_{0}$ as the initial density $n_{0}$ is varied. At low values of $n_{0}$, the compression is high, the dwell time is short, and the decrease in line density results in lower thermonuclear yields. For larger values of $n_{0}$ the final compressed temperature decreases and again the thermonuclear yield decreases. Throughout this process the complications of axial and radial thermal conduction, radiation 

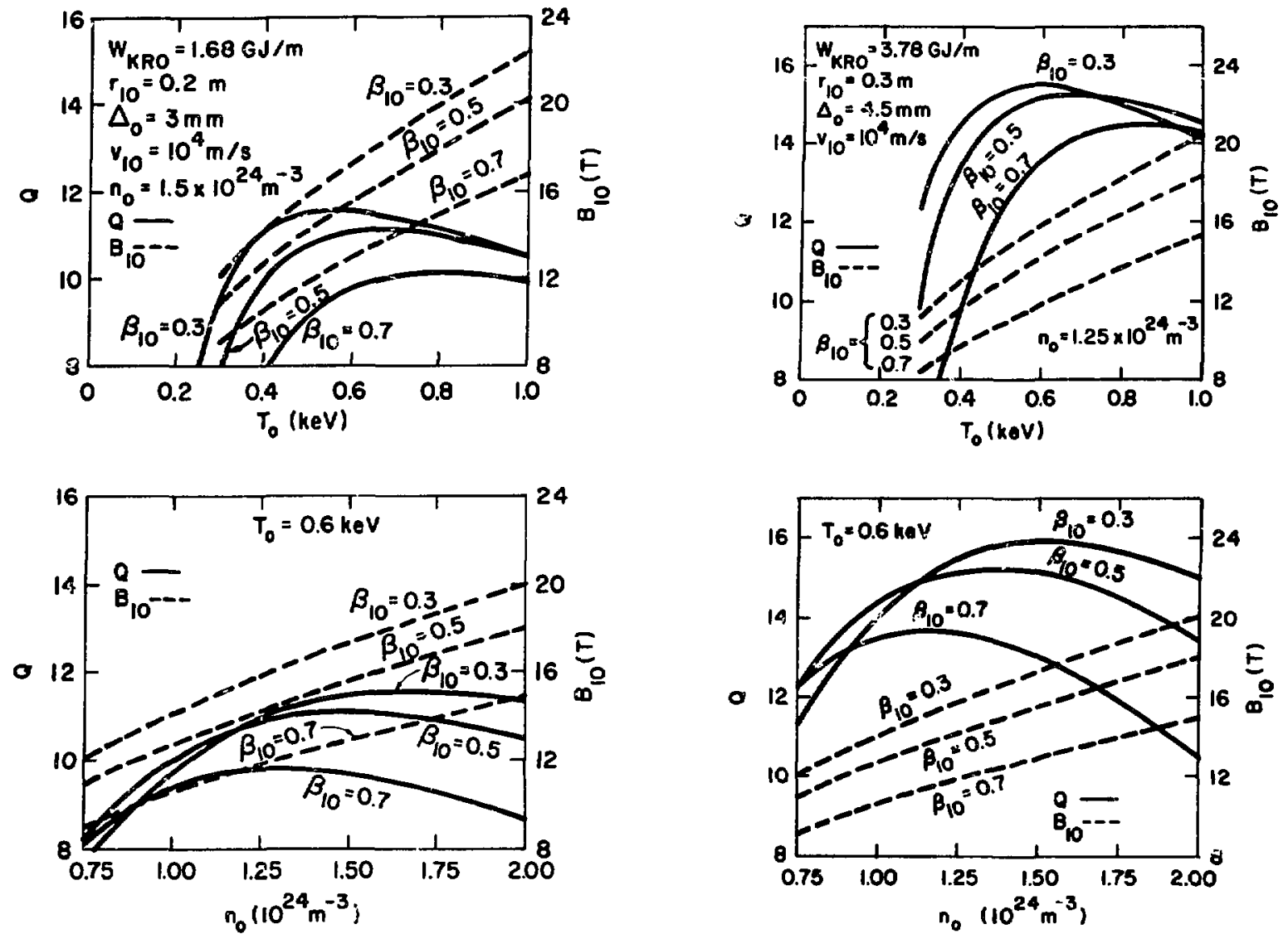

Fig. III-4. Dependence of the liner

Fig. III-5. Dependence of the liner $Q$-value $Q$, and the insulating field at the wall $\mathrm{B}_{10}$, on initial plasma temperature $T_{0}$ and density $n_{0}$. These curves have been computed for the lowyield case with $W_{K R O}, r{ }_{10}$, and $v$ as shown and global beta values at the wall of $B_{10}=0.3,0.5$, and 0.7 .

$Q$-value $Q$, and the insulating field at the wall, $B_{10}$, on initial piasma temperature $T_{0}$ and density $n_{0}$. These curves have been computed for the highyield case with $W_{K R O}, r_{10}$, and $v$ as shown and global beta values at the wall of $B_{10}=0.3,0.5$, and 0.7 . 
loss, and liner comipression play varyingly important roles. For example, Fig. III-4 shows $Q$ plotted along two orthogonal lines in $\left(T_{0}, n_{0}\right)$ space $\left(n_{0}=\right.$ $\left.1.5(10)^{24} \mathrm{~m}^{-3}, T_{0}=0.6 \mathrm{keV}\right)$. For $\beta_{10}=0.5$ the intersection of these lines closely approaches the peak of a three-dimensional "hill" at $Q_{\max }=$ 11.1. Simple extrapolation indicates that for $B_{10}=0.3, Q_{\max }=11.6$ at $T_{0}=0.5 \mathrm{keV}$ and $n_{0}=1.6(10)^{24}$, and for $B_{10}=0.7, Q_{\max }=9.9$ at $T_{0}=0.8 \mathrm{keV}$ and $n_{0}=1.3(10)^{24} \mathrm{~m}^{-3}$.

The optima determined up to this point are based on a fixed liner velocity, although two liner energies were considered. Before a trajectory in velocity space can be constructed, values of $\beta_{10}, n_{0}$, and $T_{0}$ must be selected that are technologically achievable insofar as a final reactor operating point is concerned. A low value of $\beta_{10}$ would be desirable, since the associated high fields at the wall provide good thermal insulation. Based on a qualitative judgment as to the maximum initial field (and current) that can be achieved, $\beta_{10}=0.5$ was chosen. The associated values of $n_{0}$ and $T_{0}$ for both energy cases depicted on Figs. III-4 and III-5 were selected slightly to the left of the point of optimum $Q$ in order to reduce the plasma injection requirements while still preserving a near optimum Q. For both liner energy cases the chosen values of $n_{0}$ and $T_{0}$ are depicted on the plots of $Q$ versus initial liner velocity $v_{10}$, given in Fig. III-6; the effects of liner compression and plasma losses (radiation and conduction) are also shown.

For the case of an incompressible liner and a lossless plasma shown in Fig. III-6, $Q$ drops with increasing initial liner velocity $v_{10}$ at constant liner energy (thinner liners) because of a decreased burn time. When the liner compressibility is included, but the plasma remains lossless, very low velocities require thick liners (constant initial energy), and an appreciable fraction of the initial liner energy is involved in compressing the liner material. As expected, the incompressible case is retrieved for thin fast liners. The inevitable optimum in $Q$ for compressible liners is higher than the Q-value for the incompressible liner because of the longer burn times that can result from liner compression; this increased burn time ircreases the thermonuclear yield to an extent that overcomes the assiciated liner compression losses. For the case of a compressible liner with conduction and radiation losses, the plasmla losses can become significant when the implosion and burn times are long, thereby reducing substantially the plasma energy at 


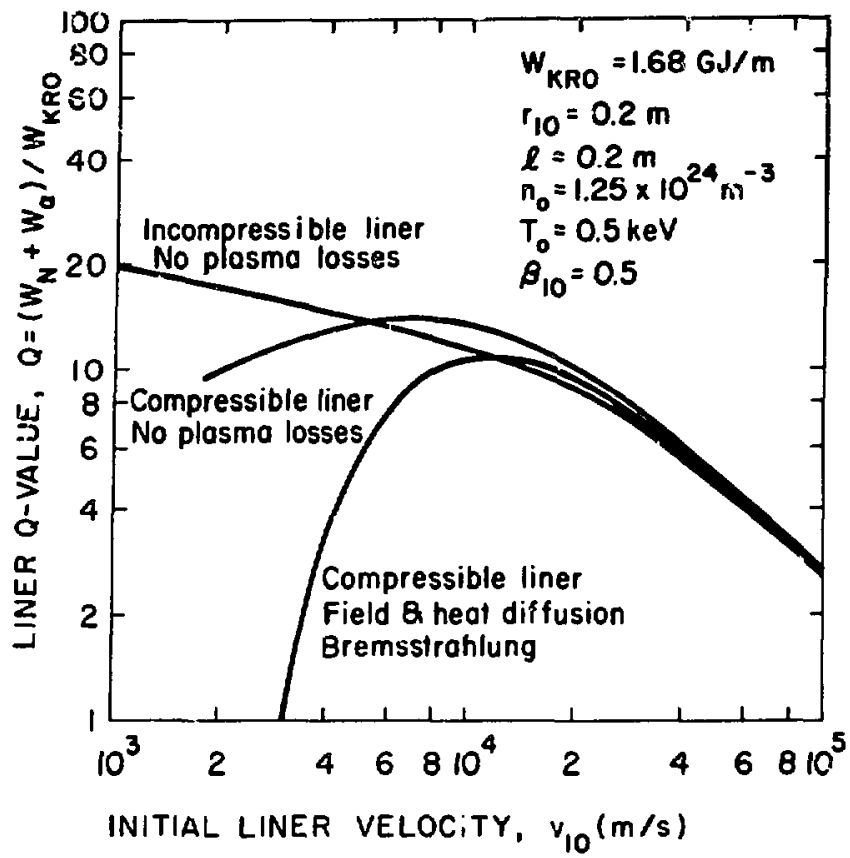

Fig. III-6A. Dependence of liner $Q$-value, $Q$, on the initial liner velocity $v_{10}$ for the near optimum cases shown on Fig. III-4. The effects of plasma losses and liner compressibility are illustrated.

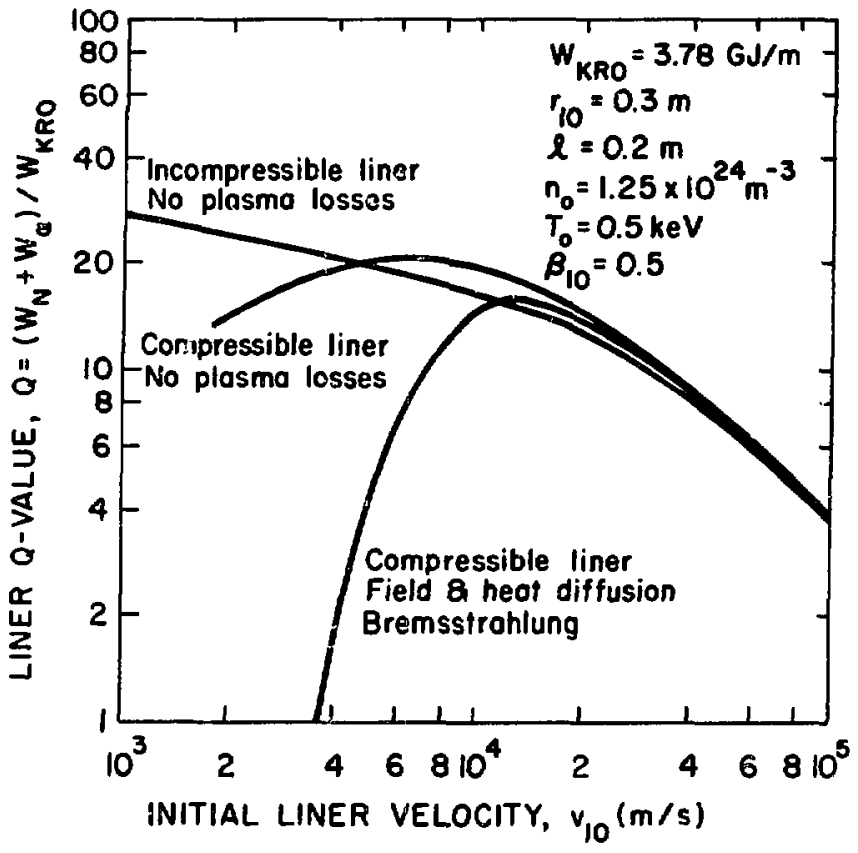

Fig. III-6B. Dependence of liner $Q$-value, $Q$, on the initial liner velocity $v_{10}$ for the near optimum cases shown on Fig. III-4. The effects of plasma losses 10 and liner compressibility are illustrated. 
peak compression. This behavior has been depicted in Fig. III-3, which shows that most losses occur during the short burn time. As the liner velocity is increased, $Q$ increases and exceeds the Q-value predicted for the losslessplasma, incompressible-liner case because of the previously described damped liner motion. At high liner velocities, the dependence of $Q$ on $v_{10}$ becomes identical to the incompressible-liner, lossless-plasma case. The optimum velocities are very close to the $v_{10}$ values assumed in arriving at the near optimum values of $n_{0}$ and $T_{0}$ in Figs. III-4 and III-5. The optimum $Q$ can be shifted to different values of $v_{10}$ by changing initial conditions $T_{0}$ and $n_{0} \cdot 24$

Based on these trade-off studies, two near-optimum FLR design points have been identified in order to pursue scoping calculations of the relevant reactor technology and economics. These interim design points are, first, the "low-yield" case, which relaxes the ETS and blast-confinement constraints but may not be attractive from the viewpoint of economics; the "high-yield" case roverses this emphasis. The essential features of these operating points are summarized in Table II-I. For both cases $v_{10}=10^{4} \mathrm{~m} / \mathrm{s}, \beta_{10}=0.5$, $T_{0}=0.5 \mathrm{keV}, \mathrm{n}_{0}=1.25(10)^{24} \mathrm{~m}^{-3}$, and $\ell=0.2 \mathrm{~m}$. Other parameters, such as the neutron energy multiplication $M=1.1$, the thermal conversion efficiency $\eta_{T H}=0.4$, the external ETS efficiency ${ }_{n_{T}}^{E X T}$, the internal (leads) ETS efficiency ${ }_{n}^{I N T}$, the fraction of the ETS energy $W_{E T S}$ needed for plasma preparation $f_{P O}=0.04$, and the similar fraction $f_{A U X}=$ 0.06 associated vith auxiliary power requirements, depend on the overall FLR system energy balance. This aspect of the FLR study is addressed in the following section.

\section{B. Reactor Engineering/Technology}

Aside from the energy transfer and storage (ETS) requirements, the FLR power sjstem portends the overall simplicity of "a pot, a pipe, and a pump." Similar to the FLR physics, however, the engineering technology in most respects is not conventional and represents an extrapolation, despite an inherent simplicity and compactness. Key technological and economic issues envisaged for the FLR are summarized below in the context of the overall FLR power system. The more crucial technological issues have been quantified where possible, although the level of effort devoted to FLR engineering has not permitted a detailed, self-consistent design. After describing the 
engineering energy balance upon which the point design summarized in Table II-I is based, the following technologies are addressed in order of perceived importance and/or difficulty: plasma preparation, ETS, liner leads, neutronics, containment, and heat transfer.

1. Energy Balance. The FLR energy balance is described schematically on Fig. III-7. The total energy transferred from the ETS system is $W_{E T S}=$ $w_{L} / n_{T}^{I N T} n_{T}^{E X}$, where $n_{T}^{E X}$ is the efficiency of energy transfer from the ETS system to the containment vessel. The electrical energy entering the containment vessel is $w_{L} / \eta_{T}^{I N T}$; of this energy $w_{L}\left(1 / \eta_{T}^{\text {INT }}-1\right)$ is dissipated ohmically in the connecting leads within the containment vessel, and $W_{L}$ reaches the liner itself. The fusion yield is composed of the neutron energy $W_{N}$ and the alphaparticle energy $w_{\alpha}$. Neutron energy multiplication in the sprayed "blanket" (coolant, blast mitigation, tritium breeder) increases the effective neutron energy to $M W_{N}$.

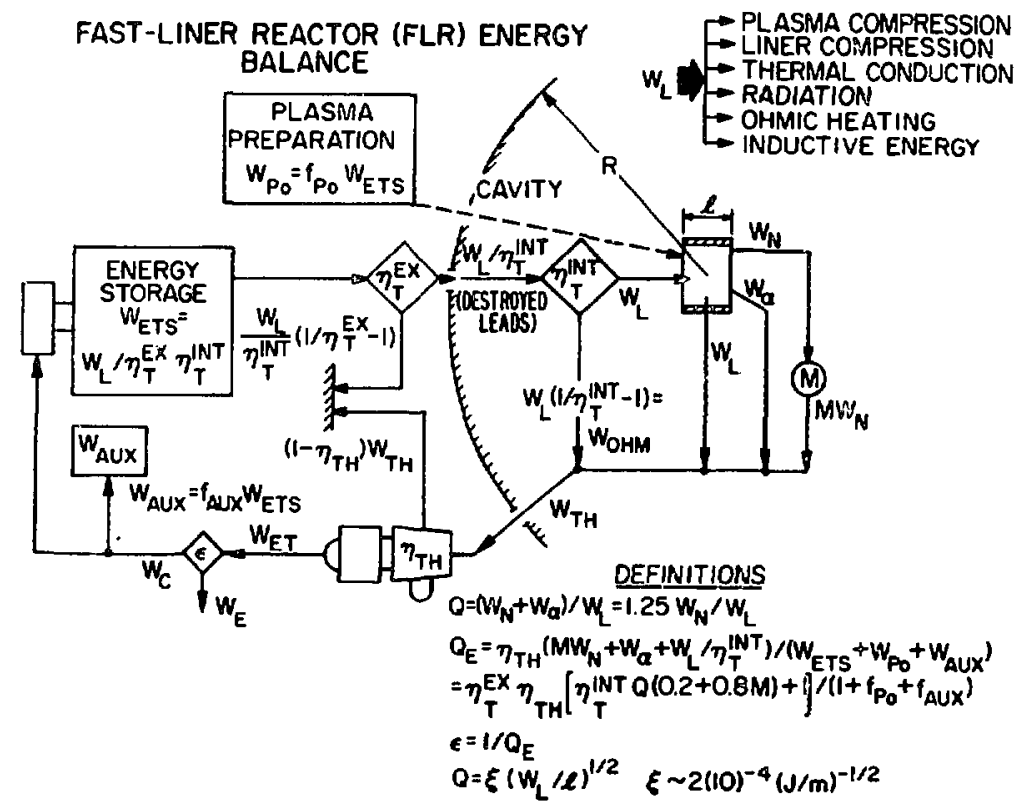

Fig. III-7. Schematic diagram of Fast-Liner Reactor energy balance, showing the partition of the energy that is delivered to the liner among the various liner energy loss mechanisms. Shown also is the relationship between the plasma or liner Q-value $Q$, and the engineering Q-value $Q_{E}$. The external transfer efficiency $\eta_{T}$ is assumed to be 0.95 and the internal transfer

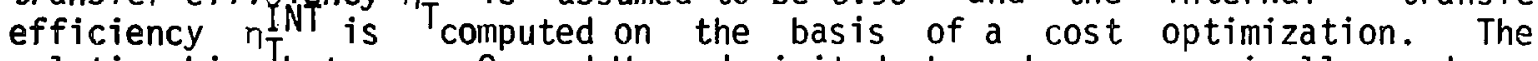
relationship between $Q$ and $W_{L}$ depicited has been numerically and analyticaliy. 17 
After each shot the high-grade thermal energy removed from the containment vessel is $W_{T H}=W_{L} / \eta_{T}^{I N T}+M W_{N}+W_{\alpha}$. This energy is converted to electricity with a thermal conversion efficiency $\eta_{T H}$.

The liner or "scientific" Q-value is defined as $Q=\left(W_{N}+W_{\alpha}\right) / W_{L}=$ $1.25 W_{N} / W_{L}$. The liner Q-value depends primarily on physics considerations of liner performance, as discussed in Sec. III.A.4, and has accordingly been "optimized." In contrast, the engineering Q-value, $Q_{E}$, measures the total electrical energy produced as compared to the energy required to operate the plant. That is

$$
Q_{E}=r_{T H}\left(M W_{N}+w_{\alpha}+w_{L} / n_{T}^{I N T}\right) /\left(w_{E T S}+w_{P O}+w_{A U X}\right)
$$

where the plasma preparation energy is $W_{P O}$, and $W_{A U X}$ is the auxiliary plant requirement. Defining $f_{P O}=W_{P O} / W_{E T S}, f_{A U X}=W_{A U X} / W_{E T S}$, and $W_{L} / W_{E T S}=n_{T}^{E X_{T} I N T}$ leads to the following relationship between $Q_{E}$ and $Q$

$$
Q_{E}=\eta_{T}^{E X} \eta_{T H}\left[\eta_{T}^{I N T}(0.2+0.8 M) Q+1\right] /\left[1+f_{P O}+f_{A U X}\right] .
$$

For the conditions depicted on Table II-I, $f_{P O}=0.04$, and $f_{A U X}$ is taken to be 0.06 . The description for a 40-50 vol\% lithium spray in Sec. III.B.5 indicates that $M=1.1$, ard for all computations $\eta_{T H}$ is taken to be 0.4 . The high-yield case (Table II-I) gives a physics-optimized $Q$ of 14.7 , whereas the low-yield baseline case selected for the tchnology assessment gives $Q=$ 10.7 The internal and external ETS transfer efficiencies, $n \underset{T}{\mathrm{~T}}$ and $n_{T}^{T N T}$, remain to be specified. It is noted the reversible recovery of the ETS energy is not required by the FLR concept.

The ETS system must supply $W_{E T S} \simeq 400 M J$ in $20-30 \mu s$ with high efficiency
$\eta_{T}$ to the containment veise1. This external circuitry would be cycled millions of times each year, and considerable flexibility and expense would be evoked to assure that $\eta_{T}{ }^{\circ} \gtrsim 0.95$ could be achieved; the parasitic energy $W_{E T S}\left(1-n_{T}\right)$ generally represents both a loss in revenue as well as 
added capital expenditures needed for the incremerital ETS system. In contrast, a major portion of the leads structure located within the containment vessel would be destroyed each shot; the configuration of these internal leads determines the ultimate value of $\mathrm{n} \mathrm{INT}_{\mathrm{T}}$. Hence, the design values of $\eta_{T}^{I N T}$ must be determined by an optimization procedure that balances the cost of destroyed leads structure, leads energy loss (recovered by the thermal cycle), and the effects on plant revenue/cost as reflected by the dependence of $Q_{E}$ on $n_{T}^{I N T}$ (Eq. (III-18)). This latter issue is addressed in Sec. III-B.4; generally $n_{T}^{\text {INT }} \approx 0.9$ is required. On this basis, Table II-I indicates $Q_{E}=3.94$ for the low-yield case, which corresponds to a recirculating power fraction $\varepsilon=1 / Q_{E}=0.25$.

2. Plasma Preparation. According to Table II-I for the low-yield case, the optimized initial plasma requirements are $T_{0}=0.5 \mathrm{keV}$, $\mathrm{n}_{0}=1.25(10)^{24} \mathrm{~m}^{-3}$, and an initial azimuthal magnetic field at the wall $B_{0} \simeq 13 T\left(B_{10} \simeq 0.5\right)$; these parameters correspond approximately to $3.4 \mathrm{MJ}$ of plasma energy delivered to the $\sim 0.025-\mathrm{m}^{3}$ initial liner volume $\left(r_{10}=0.2 \mathrm{~m}, \ell=0.2 \mathrm{~m}\right)$ on a $\sim 1-\mu \mathrm{s}$ time scale. The field energy corresponds to $\sim 1 \mathrm{MJ}$, which for a uniform current density amounts to $100 \mathrm{MA} / \mathrm{m}^{2}$ or $13 \mathrm{MA}$. Four potential plasma-preparation schemes are under consideration: coaxial gun injection, ${ }^{25}$ shock-tube injection, ${ }^{26}$ exploding D-T threads, 27 and in situ plasma formation by 1 aser 28 or relativistic-electron ${ }^{29}$ beams. As an example of the first case, a coaxial gun would be located outside the blast zone to inject the plasma along a magnetic guide field to the liner. The guide tube and field would be located inside the liner/lead structure. Plasmas have been produced with densities of $2(10)^{23} \mathrm{~m}^{-3}$ and directed energies of $\approx 0.2 \mathrm{keV}$, and these plasmas are believed to contain embedded poloidal fields. ${ }^{25}$ Substantial development is required, however, to create plasmas at the temperature, density, and field required by the FLR. The problem of transporting such a plasma is also unsolved.

The electromagnetic shock-tube and exploding-wire techniques would produce the plasma inside the liner, thereby eliminating the need for transport from an external source. The plasma source in this case must be simple and inexpensive, since it must be replaced by each shot. For the case of the electromagnetic shock tube, a high current passing though a conductor along the liner axis produces a strong poloidal field near the conductor. The short 
field risetime ( $\sim$ $1 \mu \mathrm{s}$ ) causes an electromagnetic shock to propagate radially from the conductor, heating the surrounding DT gas to the required plasma temperature. Plasmas with $0.5-\mathrm{keV}$ temperatures and $\sim 10^{22}-\mathrm{m}^{-3}$ densities have been produced by this method; much more work, however, is needed to reach the projected reactor parameters.

An example of the exploding-wire technique would have a solid (cryogenic) of filament placed along the liner axis. A strong axial current would cause the thread to explode and to form the required plasma in situ. Deuterium threads with the appropriate dimension (300- $\mu \mathrm{m}$ diameter) have been produced, ${ }^{27}$ but whether the necessary plasma condition can be reached must still be demonstrated. Like the shock-tube and DT filament approaches, laser or electron beams could produce the required temperatures and densities in situ. The use of beams, however, could eliminate the need for delicate or expensive apparatus that must be located in the vicinity of the liner. $\mathrm{CO}_{2}$ laser beams have routinely produced the required $\sim 0.5-\mathrm{keV}$ temperatures at $\sim 10^{24} \mathrm{~m}^{-3}$ density, ${ }^{28}$ but these plasmas have not been produced in the required volumes $\left(0.025 \mathrm{~m}^{2}\right)$. Although the question of beam transport remains for the relativistic-electron-beam approach, the generation of the required insulating magnetic fields may be more straightforward than for laser beams.

In sumary, both the theoretical and experimenial state of the art for FLR plasma preparation is embryonic but developing. Although more computation can be made on the various techniques suggested above, detailed design of this aspect of the FLR is expected to remain vague until related experiments are performed. The primary contribution that this systems study can make at this point in the development of fast-liner fusion is to quantify from the reactor viewpoint the optimal initial condicions $\left(n_{0}, T_{0}, B_{10}\right)$ and to indicate the consequences of not achieving these aptimal conditions in the laboratory (i.e., Fig. III-5). Plasma preparation is viewed as one of the more crucial physics and technological issues for the FLR concept, and consequently, is being subjected to early experimental study. ${ }^{19}$

3. Energy Storage, Switching, and Transfer. The liner drive in a typical, low-yield FLR (Table II-I) will require $\sim 250 \mathrm{MA}$ at $\sim 200 \mathrm{kV}$ in 200-300 $\mathrm{s}$ for an energy transfer of $450 \mathrm{MJ}$. This energy transfer $W_{E T S}$ is $\sim 10 \%$ greater than deduced from Table II-I in order to account for resistive losses in the liner, which are not included in the LNRBRN model (Appendix A). 
Both inductive and capacitive energy storage were considered in a preliminary study of a much lower yield FLR ${ }^{5}$ ( $\left.W_{L} \simeq 70 \mathrm{MJ}\right)$; Figure III-8 schematicaliy depicts these two ETS options. In the inductive ETS system a homopolar generator would transfer energy to a normal conducting inductor in a few milliseconds; current would then be switched to the liner, transferring energy on a 20-us time scale. For a capacitive ETS a large capacitor bank would be discharged directly to the liner.

Although inductive energy storage is considerably less expensive ${ }^{30}$ than comparable capacitors, inductive ETS nevertheless requires a substantial transfer capacitor to eliminate resistive energy losses incurred during the transfer and to couple efficiently the source (ETS) and load (liner). In preliminary FLR studies ${ }^{5} 45 \%$ of $W_{L}$ was held in the transfer capacitor at the end of the transfer cycle. An inductive system, therefore, would show little advantage compared to a capacitive ETS unless the required transfer capacitor could be made substantially smaller than the total energy storage.

Another advantage of capacitive energy storage arises because switches must only close a circuit during a given cycle rather than requiring high-current opening or interrupting switches. Although the switching problem has not been thoroughly studied for the FLR application, the magnitude of both power and energy transfer is far beyond the capability of the present commercial sector. Without a considerably more detailed study, the cost of switching on this scale cannot be predicted accurately.

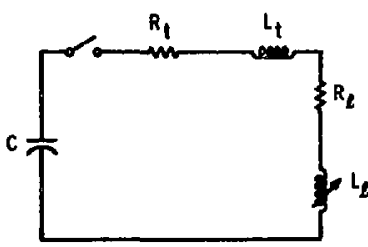

C EFFECTIVE tTS CAPACITANCE

R. RESISTANCE OF TRANSFER LINES

$L_{t}$ INDUCTANCE OF TRANSFER LINES

$R_{\ell}$ RESISTANCEOF LINER

$L_{\ell}$ INDUCTANCE OF LINER

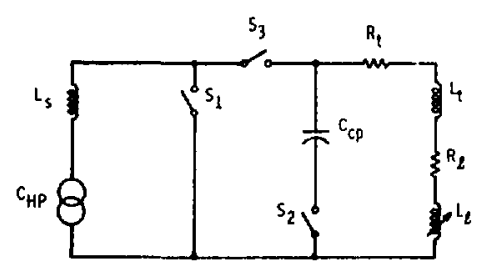

$C_{H P \text { CAPACITANCE OF HOMOPOLAR MOTOR / GENERATOR }}$

$c_{c p}$ COUNIERPULSING AND TRANSFER CAPACITOR

Ls STORAGE INOUCTOR

$R_{q}$ RESISTANCE OF TRANSFER LINES

$l_{t}$ INDUCTANCE OF TRANSFER LINES

$R_{L}$ RESISTANCE OF LINER

$L_{l}$ INOUCTANCE OF LINER

Fig. III-8. Schematic diagrams of capacitive and inductive energy transfer and storage (ETS) systems being considered to drive the FLR liner implosion. 
Considering the magnitude of ETS power requirement, the nature of transmission lines should be reexamined. A new interleaved liner leads structure is described in the following section for energy transfer inside the containment vessel. A similar conductor could be envisaged for the permanent external circuitry to provide a compact, low-inductance carrier superior to coaxial cables.

4. Liner Leads. During the initial formulation of the FLR concept $^{5}$ it was assumed that electrical power would be transferred to the liner by a coaxial lead structure or perhaps by circular parallel plates separated by an insulator; these leads concepts are shown schematically in Fig. III-9. Several reasons were subsequently identified that make these approaches unattractive, if not unacceptable, for an FLR.

Typica77y, the liner must be supplied with 250 MA at $200 \mathrm{kV}$ for 20

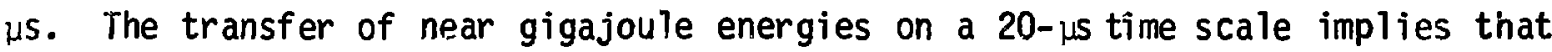
lead inductances between the ETS/switching system and the liner must be smal1; unacceptable amounts of parasitic energy would otherwise be stored, increasing
A.
B.
C.

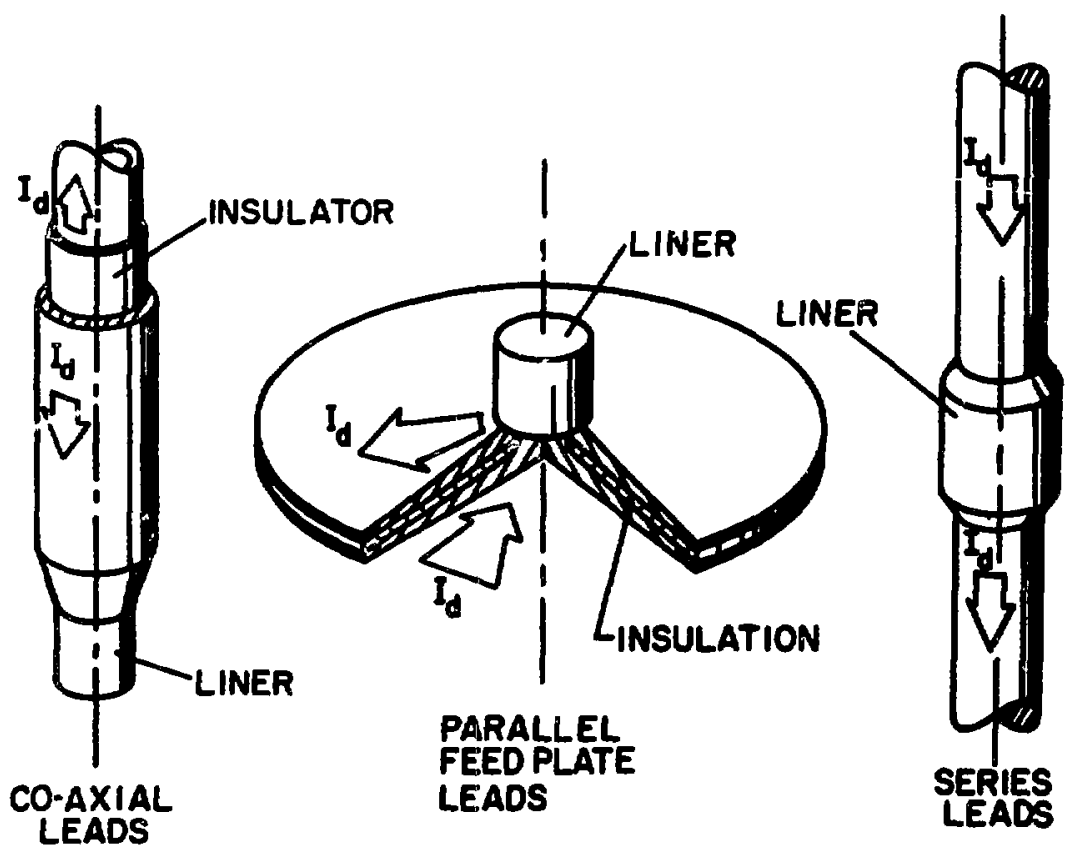

Fig. III-9. Schematic diagram of a range of possible leads configurations using a general coaxial approach. Cost optimization of this general class of leads structure is given in Appendix B. 
the ETS energy and voltage requirements. In order that parasitic inductances be maintained small compared to the liner inductance, the distance separating the two conductors must be as small as possible to reduce field energy between conductors. These constraints largely eliminated the concepts shown in Fig. III-9-C, where separate probes of opposite polarity enter the confinement chamber from opposite directions and converge on the liner. One exception would incorporate the switch in the shape of a cylinder that encases the liner at its outer surface. Current would build up in the probes and eventually would be switched during a long purse; in this case the confinement cavity itself would act as a magnetic energy storage element. The switch around the liner would then be opened and would quickly transfer current to the liner. To date, however, no switch has been conceived that could sustain the high currents and forces required for this approach.

Three additional problems can be identified .ith the simple coaxial approach depicted in Fig. III-9-A. First, unless the conductors are large and massive, magnetic field pressure between the conductors lould rapidly drive the conductors apart, thereby dissipating a substantial fraction of the input power as leads kinetic energy. Second, the optimum leads radius can be computed, which minimizes the expense of recycling conductor and insulator mass as well as energy losses associated with both joule heating and acceleration of the conductor. Generally, as shown in Appendix B, the optimum radius is inconveniently large in comparison to the desired size of the containment vessel. Third, at a point $\sim 2 \mathrm{~m}$ from the liner, the leads structure that is normally destroyed must be connected to an input conductor that is designed to survive the explosive forces attendant to the liner implosion. A coaxial conductor of radius $r_{c_{2}}$ and carrying a current $I_{d}$ encounters a field pressure of $\mu_{0} I_{d}^{2} / 8 \pi^{2} r_{c}^{2}$. Taking the yield strength of steel to be $\sim 400 \mathrm{MPa}(58 \mathrm{kpsi})$ and $I_{d}=250 \mathrm{MA}$, the reusable coaxial conductor must have a radius in excess of $1.6 \mathrm{~m}$, which is an unreasonably large value and generally is not compatible with the optimization results given in Appendix $B$. From the viewpoint of energy and materials cost the radial feedplates shown in Fig. III-9-B would be more desirable than the coaxial conductors described above. Serious problems related to joule heating and conductor motion, as well as the difficulty of rapidly handing such an object still exist. These issues are addressed quantitatively in Appendix $B$. 
The overall leads size can be greatly reduced and serious conductor motion alleviated if the interleaved lead structures shown in Fig. III-10 are used. In the interleaved leads concept alternate conductors carry current to and from the liner; insulation, shown in Fig. III-11, is woven between conductors of opposite polarity. The conductor thickness in the azimuthal direction should be no thicker than twice the skin depth, $\Delta=\sqrt{2 n \tau / \mu_{0}}$, for a pulse length $\tau$ and resistivity $n$. The radial thickness $\Delta r=r_{0}-r_{j}$ of the conductor is determined by a trade-off between Joule-heating costs ( $\propto 1 / \Delta r$ ) and material costs $(\propto \Delta r)$. Appendix $C$ describes a quantitative treatment of this optimization between energy and materials costs. As noted in Sec. III.B.1, it is this cost optimization that primarily determines the internal transfer erfficiency, $n_{T}$ INT.

The inter leaved conductor can be compared to the coaxial leads through an apparent radial field pressure on each conductor. The ccaxial conductor has a field pushing the two conductors apart with a pressure

$$
P_{c l} \simeq \mu_{0} I^{2} / 8 \pi^{2}\langle r\rangle^{2},
$$

where $\langle r\rangle$ is the average of the two conductor radii. If the interleaved conductors are wider in the radial direction so that $\Delta r=r_{0}-r_{j}>\Delta$, it can be shown that the average outward radial pressure on the conductors is given by

$$
P_{i \ell} \simeq \mu_{0} I^{2} / N^{2}\left(r_{0}^{2}-r_{i}^{2}\right) \simeq r_{0} I^{2} / 2 N^{2}(\Delta r)<r>
$$

where $N$ is the total number of conductors. The conditior $r_{0}-r_{j}>\Delta$ implies $N\left(r_{0}-r_{j}\right)>\pi\left(r_{0}+r_{j}\right) \equiv 2 \pi<r>$. This condition leads to an upper bound on the interleaved conductor pressure given by

$$
\left.P_{i \ell} \leq \mu_{0} I^{2} / 4 \pi N<r\right\rangle^{2}
$$


FORCE-FREE FLR LEAUS STRUCTURE

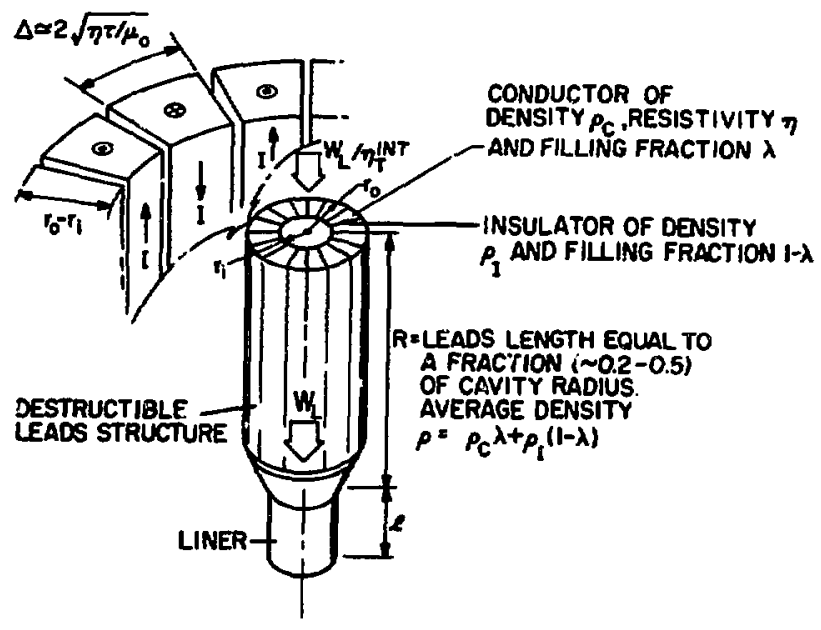

Fig. III-IO. Schematic diagram of "force-reduced" interleaved leads structure (Fig. III-1) showing extruded conductor material that would be destroyed each shot and become part of the primary coolant ( $\mathrm{Li}$ or $\mathrm{LiPb}$ ). The energy transfer to the liner per se, $W_{L}$, is transferred with an efficiency $n_{T}{ }^{N T}$ from the containment vessel feedthrough. This geometry and the value of $\eta_{T}^{T N T}$ is determined on the basis of optimal costs (Appendix C).

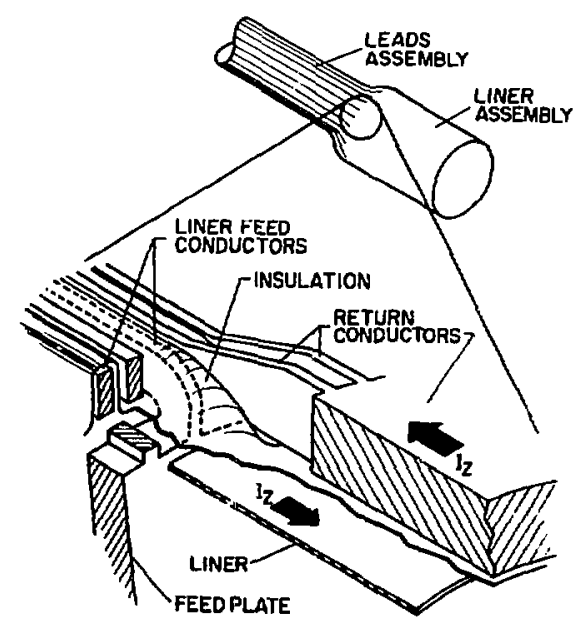

LEADS STRUCTURE FOR FLR

Fig. III-11. Detailed view of attachment of "force-reduced" interleaved leads structure to the liner. 
The radial pressures on the two systems are related as follows

$$
P_{i \ell}<(2 \pi / N) P_{c \ell},
$$

when the average radii for both the coaxial and interleaved systems are equal. Typically, $\mathrm{N}$ can be made on the order of 100 , and the radial pressure on interleaved leads, therefore, can be made negligible when compared to coaxial leads. Likewise the kinetic energy imparted to interleaved leads would be insignificant.

The interleaved leads approach eliminates the leads kinetic energy as a design constraint, and the crucial constraints become the costs of ohmic heating (i.e., added ETS requirement or decreased $n_{T}^{\text {INT) }}$ ) and materials destruction (i.e., cost of materials fabrication and replacement). Although this cost optimization is described in detail in Appendix $C$, given below is a brief description of the energy versus materials trade-off for the "force-reduced," interleaved leads concept.

The resistance $R_{L}$ per unit length of leads is given by (Fig. III-10)

$$
R_{L}(0 h m / m) \simeq 4 n / N \Delta\left(r_{0}-r_{j}\right)=2 n\left(\Delta+\Delta_{I}\right) / \pi \Delta<r>\left(r_{0}-r_{j}\right),
$$

where $\Delta$ must be twice the skin depth, and $\Delta_{I}$ is the insulator thickness. Assuming a sinusoidal pulse, the ohmic heating per meter of lead length is

$$
W_{O H M} \simeq R_{l} I_{d}^{2} \tau / 2 \simeq n\left(\Delta \Delta_{I}\right) I_{d}^{2} \tau / \pi \Delta<r>\left(r_{0}-r_{j}\right)
$$

The conductor mass requirement per meter of lead is given by

$$
M_{C}=2 \pi \rho_{c} \Delta<r>\left(r_{0}-r_{i}\right) /\left(\Delta+\Delta_{I}\right),
$$


and the mass of the leads insulator is given by

$$
M_{I} \simeq 2 \pi \rho_{I}<r>\Delta_{I}\left[1+\left(r_{0}-r_{i}\right) /\left(\Delta+\Delta_{I}\right)\right],
$$

where $\rho_{C}$ and $\rho_{I}$ are conductor and insulator densities. Cost factors can be assigned to $W_{O H M}, M_{C}$, and $M_{I}$, and the radial thickness $\left(r_{0}-r_{j}\right)$ can be adjusted to minimize the total leads cost; this optimization is described in Appendix $C$. It should be noted that within reasoriable limits, the average radius, $\langle r\rangle$, can be fixed, and an optimization can be performed with respect to $r_{0}-r_{j}$; it is no longer necessary to optimize using the overall size, as in the cost of coaxial conductor (Appendix B).

Figure III-12 gives the results of the simple cost optimization described in Appendix $C$. Under the assumption that materials costs are not incurred for the leads conductor beyond the initial capital investment and that the

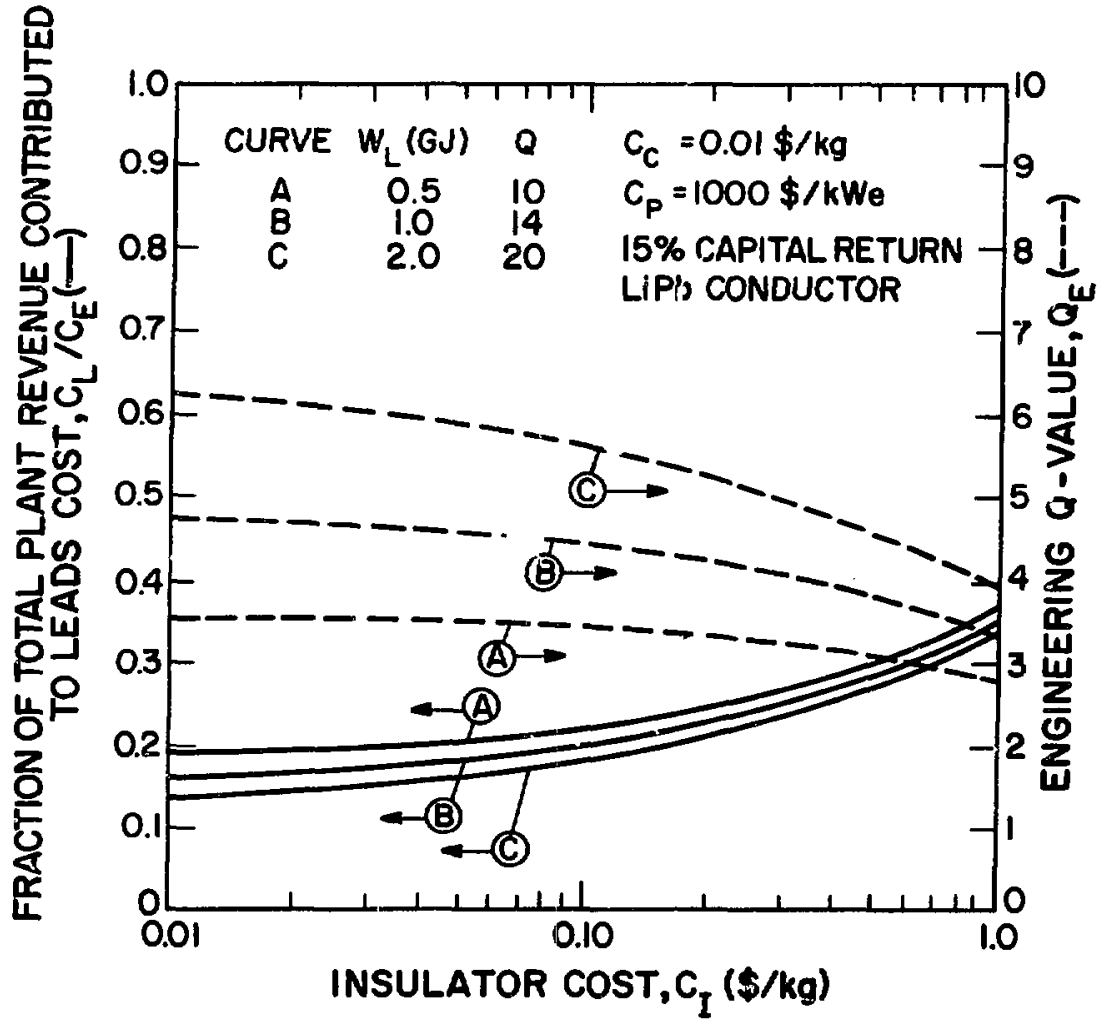

Fig. III-12. Dependence of leads cost $C_{L}$ (relative to net power cost $C_{E}$ ) and the engineering $Q$-value $Q E$ on the unit cost of leads insulator $C_{I}(\$ / \mathrm{kg})$ for a leads conductor recycle cost $C_{C}$ of $0.01 \$ / \mathrm{kg}$. The liner energies $W_{L}$ and liner $Q$-value $Q$ are constrained (Re: Appendix $C$ ). 
conductor can be recovered and recycled at a cost of $0.01 \$ / \mathrm{kg}$, Fig. III-12 gives the dependence of the total leads cost, relative to the net plant reverue, on the total cost of the insulator. Figure III-12 also gives a similai dependence for the engineering $Q$-value $Q_{E}$ and a range of liner yields $\left(W_{L}, Q\right)$. In deriving these curves the relationship $Q=$ $\xi\left(W_{L} / l\right)^{1 / 2}$ has been used to relate analytically the liner Q-value and the liner energy per unit length $W_{L} / l$; the constant $\xi$ is derived from the impulse-momentum theorem and shows good agreement with the predictions of both the LNRBRN and CHAMISA codes. ${ }^{17}$ Generally, if the insulator costs can be held below $\sim 0.10 \$ / \mathrm{kg}$, the total leads cosi will be comparabie to a "fuel cost," amounting to $\sim 20-30 \%$ of the plant revenue, and the decrease in plant efficiency required to optimize the plant revenue is not significant.

The optimization procedure used to arrive at Fig. III-12 is based on an optimum cross-sectional area of the interwoven leads structure that will maximize total plant revenue (re: Appendix $C$ ). Generally, the optimum leads area is sufficiently small to cause melting sometime during the energy transfer. Appendix $\mathrm{C}$ treats the case where the leads are constrained to remain solid throughout the implosion, rather than selecting a conductor area that maximizes plant revenue. These results generally are more pessimistic than those presented in Fig. III-12 and are discussed in Appendix C.

Last, $i c$ is emphasized that the results presented here represent a local or "point" optimization that focuses on the leads and treats all other plant costs as a lumped parameter $\left(C_{p}=1000 \$ / \mathrm{kWe}\right.$ with $15 \%$ capital return $)$. Once more detailed designs are available for all crucial components, a comprehensive cost optimization must be performed.

5. Neutronics Analysis. Like other technology areas for the FLR, only scoping studies have been macie with respect to the neutronics design. The purpose of the neutronics studies performed to date is threefold: (a) to resolve the relationship between tritium breeding in the $\mathrm{Li}$ (or $\mathrm{LiPb}$ ) blanket spray that would surround the liner prior to implosion, the composition and volume fraction of the spray, and the size of the containment vesse 1 as dictated by stress considerations; (b) to determine the energy density profiles in both the Li spray and at the first structural wal1; (c) to resolve the degree to which nuclear heating occurs within the liner and leads structure and to determine the effects of this heating on the liner dynamics and the amount of destroyed leads. Because of the complex geometry associated with 
the liner leads, liner/leads penetration, and blast containment (Fig. II-1), a Monte Carlo approximation was adopted; this approach sacrifices spatial resolution for more flexibility in describing the time-dependent, asymmetric problem.

The continuous-energy Monte Carlo code MCNP ${ }^{31}$ was used for the idealized neutronics analysis. This code employs any number of cells and uses standard variance reducing techniques (optional), which include particle splitting, Russian roulette, and path-length stretching. Provisions are also made for forcing collisions in designated cells, cbtaining flux estimates at point detectors, and for calculating reactions in small regions using track-length estimates. Specification of a source particle consists of a geometry location, angular description, energy, time, and particle weight, with probability distributions being designated for any of these variables. Additional information on the MCNP calculational procedure is found in Appendix $D$.

Figure III-13 depicts the MCNP geometry used to model the liner, leads, leads penetration, lithium-spray cavity, and the containment walls; Tabie III-I identifies each region used in the neutronics approximation. A vacuum

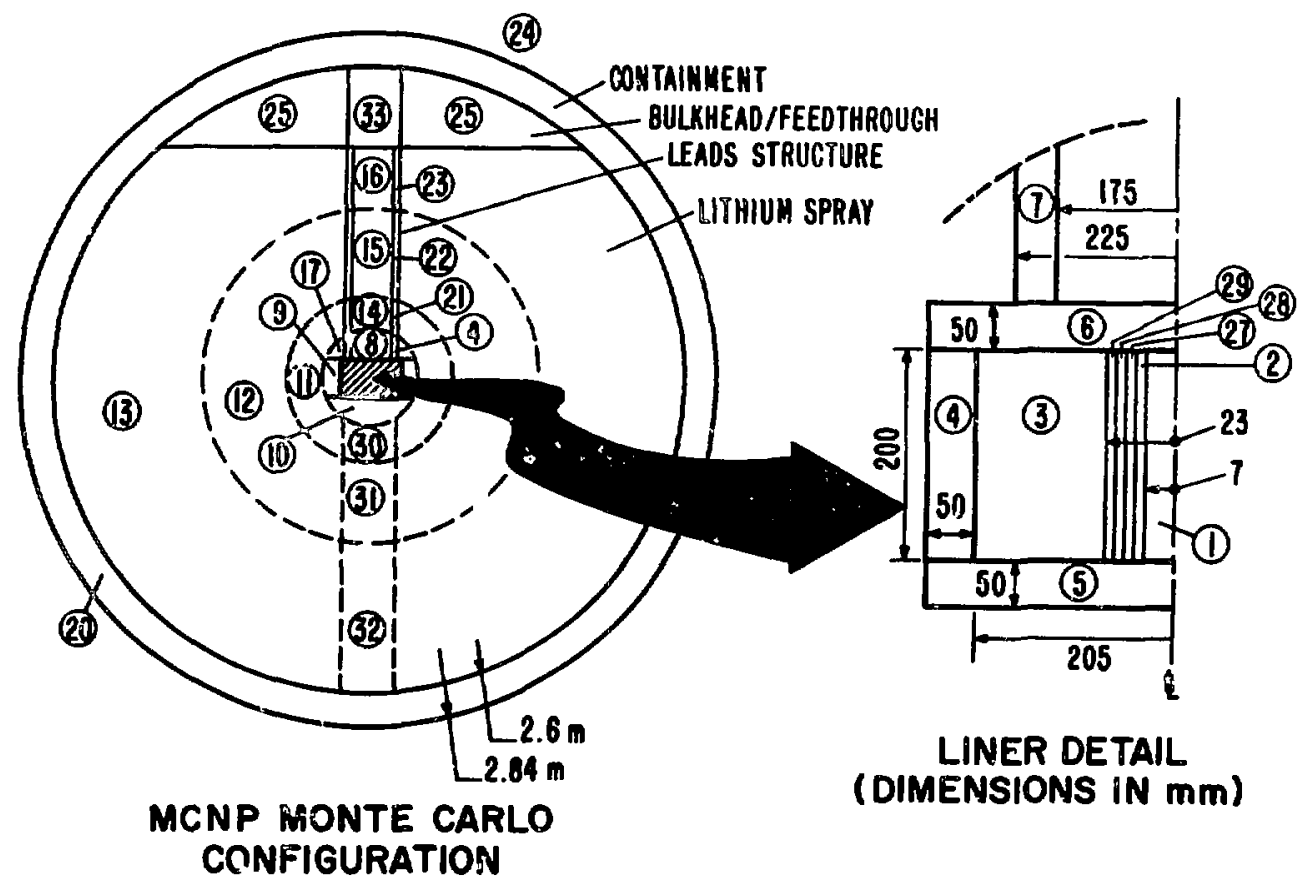

Fig. III-13. Neutronics model used to compute nuclear heating via MCNP Monte Carlo code. Refer to Table III-I for zone characteristics. 
TABLE III-I

\section{DESCRIPTION OF FLR REGIONS USED IN MCNP MONTE CARLO NEUTRONICS CALCULATION (FIG. III-13)}

\begin{tabular}{|c|c|c|}
\hline Region & Description & Composition \\
\hline 1 & Compressed Plasma & Void (Source) \\
\hline 2 & Liner & $\mathrm{Cu}$ (2.5 theoretical density) \\
\hline 3 & $-\infty$ & Void \\
\hline 4 & Return Conductor & $\mathrm{Cu}$ or $\mathrm{LiPb}$ \\
\hline 5 & Bottom Endplug & $\mathrm{Cu}$ or $\mathrm{LiPb}$ \\
\hline 6 & Top Endplug & $\mathrm{Cu}$ or $\mathrm{LiPb}$ \\
\hline 7 & Electrical Lead & $\mathrm{Cu}$ or $\mathrm{LiPb}$ \\
\hline $8-17$ & Lithium & $\operatorname{Li}(25-50 \mathrm{v} / 0)$ \\
\hline $18-19$ & No Designation & -- \\
\hline 20 & Containment Vesse? & $\mathrm{Fe}$ \\
\hline $2 ?-2.3$ & Electrical Lead & $\mathrm{Cu}$ or $\mathrm{LiPb}$ \\
\hline 24 & - & Void \\
\hline 25 & Feedthrough/Top & $\mathrm{Fe}$ \\
\hline 26 & No Designation & - \\
\hline $27-29$ & Liner & Cu (2.5 theoretical density) \\
\hline $3 T-32$ & Lithium & Li $(25-50$ vol\%) \\
\hline 33 & Feedthrough/Top & $\mathrm{Fe}$ \\
\hline
\end{tabular}

boundary condition was imposed outside the containment vessel, since shielding computations are not of primary interest here. Gamma/neutron heating and tritium breeding were computed for each Monte Carlo cell. The time-dependent 14.1-MeV neutron source from cell region [1] was generated by the LNRBRN code (Appendix A) and is depicted on Fig. III-14, which also gives the cumulative gamma-ray and neutron energy deposition for a Cu/LiPb liner assembly; for all cases the liner per se was copper. The liner configuration depicted on Fig. III-13 corresponds to condition expected at peak compression with the massive return conductors ( $\mathrm{Cu}$ or $\mathrm{LiPb}$ ) assuming the initial ( $\sim 0.2-\mathrm{m}$ radius) position. Taking the melting energy as that needed to melt a lirer starting from room temperature $\left(610 \mathrm{MJ} / \mathrm{m}^{3}\right.$ for $\mathrm{LiPb}$ and $5.92 \mathrm{GJ} / \mathrm{m}^{3}$ for $\left.\mathrm{Cu}\right)$, Fig. III-I4 indicates that melting of the liner from nuclear heating alone will probably occur prior to peak compression; this inference is approximate 


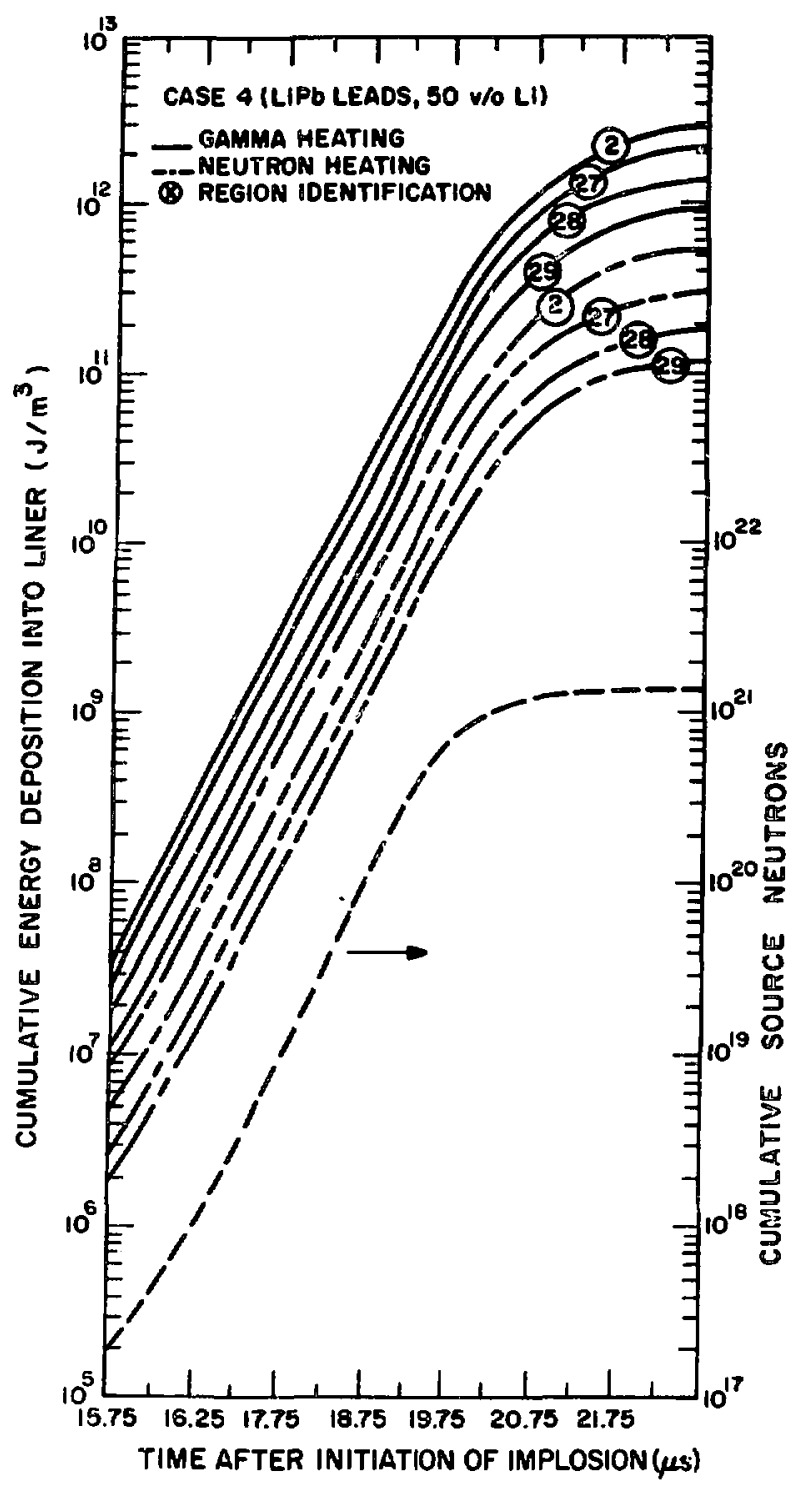

Fig. III-14. Time-dependence of integrated neutron and gamma heating in a compressed copper liner for the regions indicated (Table III-I). Shown also is the cumulative neutron source $(14.1-\mathrm{MeV})$ integrated in time. Time is measured relative to the beginning of the liner implosion.

in that MCNP does not model the liner dynamics, but pessimistically assumes a thickness and density at peak compression. Figure III-15 gives the radial distribution of total nuclear heating in a copper liner for cavity volume fractions of lithium equal to 0.25 and 0.40 . 


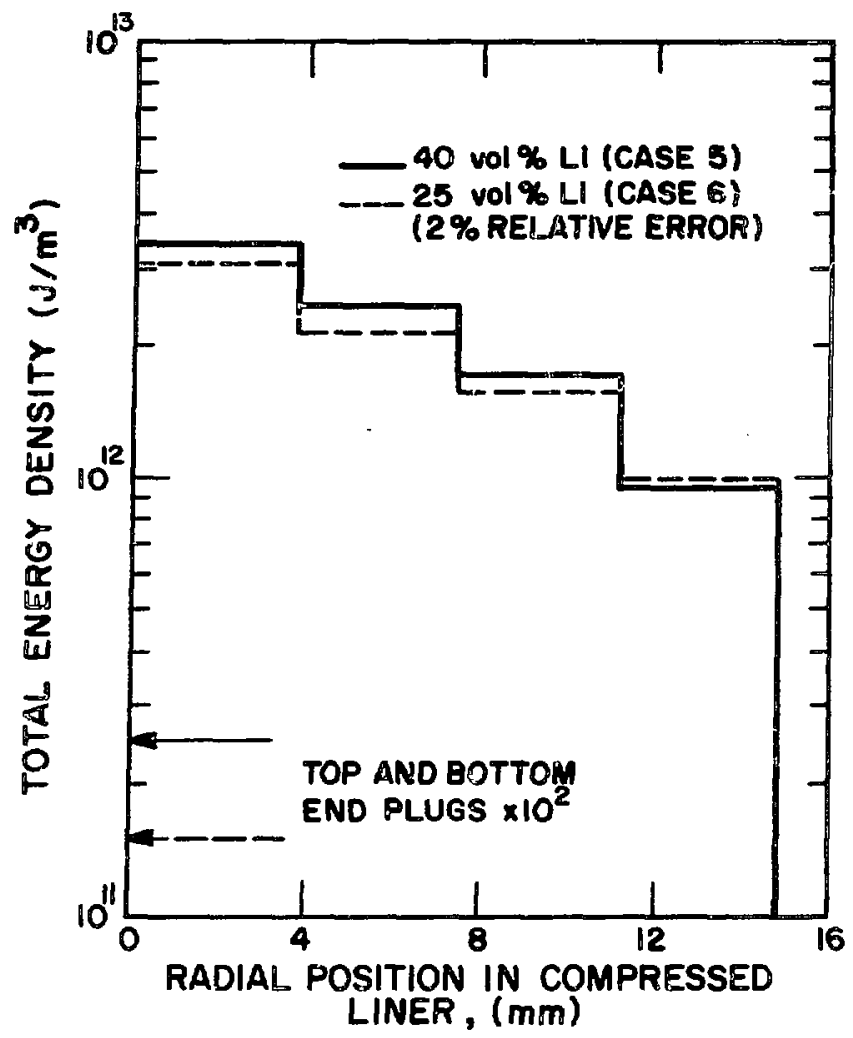

Fig. III-15. Radial distribution of total energy density deposited into a copper liner at peak compression for the volume percentages of $L i-$ spray coolant shown. The 50 vol\% case follows closely the distribution shown for the 40 vol\% case. The energy density in the top and bottom endplug region is also shown (Re: Fig. III-13).

Table III-II sumarizes the key results for the six MCNP calculations. Generally, tritium breeding in a lithium spray of volume fraction in excess of 0.25 presents no problem. As expected, the lithium spray itself is a poor energy multiplier, but some variation is seen with the liner assembly material (LiPb versus $\mathrm{Cu}$ ); this variation is seen primarily in the tritium breeding ratio and reflects the higher $(n, N n)$ reaction cross section for lead. The net number of neutron crossings at the steel vessel is also shown on Table III-II, and the lithium spray is seen to be inadequate as an efficient neutron shield/absorber for the containment ve.se?. For instance $7.8 \%$ and $14.0 \%$ of the starting neutrons leave, respectively, the outer boundary or cross the inner boundary of the steel vessel for case 4 (50 vol\% lithium in the cavity, LiPb lead/liner structure). Table III-III gives the spectrum and the track lengich per unit volume (i.e., neutron flux) at the steel containment 
vessel: this flux generally peaks in the 10-to 100-keV energy range. For a 10-s pulse rate, average neutron fluxes in the range $\sim 5(10)^{18} \mathrm{n} / \mathrm{m}^{2} \mathrm{~s}$ with this energy spectrum (Table III-III) would be expected.

TABLE IIT-II

SUMMARY OF SIX MCNP (MONTE CARLO) NEUTRONICS COMPUTATJONS MADE FOR FLR CONFIGURATIONS DEPICTED IN FIG. III-13(a)

\begin{tabular}{|c|c|c|c|c|c|c|c|}
\hline Case & $\begin{array}{l}\text { Liner/Leads } \\
\text { Material }\end{array}$ & $\begin{array}{l}\text { Lithium } \\
\text { Volume } \\
\text { Fraction } \\
\text { in Cavity }\end{array}$ & $\begin{array}{c}\text { Tritium } \\
\text { Breeding } \\
\text { Ratio }\end{array}$ & $\begin{array}{c}\text { Total } \\
\text { Neutron } \\
\text { Multiplication } \\
\end{array}$ & $\begin{array}{r}\text { Fraction } \\
\text { Starting } \\
\text { Neutrons } \\
\text { Leaving } \\
\text { System } \\
\end{array}$ & $\begin{array}{l}\text { Net Number } \\
\text { of Neutrons } \\
\text { Crossing } \\
\text { Inner Surf ace } \\
\text { of Vessel } 1020\end{array}$ & $\begin{array}{c}\text { Total } \\
\text { Energy } \\
\text { Multiplication } \\
\end{array}$ \\
\hline $\begin{array}{l}1 \\
2 \\
3 \\
4 \\
5 \\
6\end{array}$ & $\begin{array}{r}\mathrm{Cu} \\
\mathrm{Cu} \\
\mathrm{Cu} \\
\text { LiPb } \\
\text { LiPb } \\
\text { LiPb }\end{array}$ & $\begin{array}{l}50 \\
40 \\
25 \\
50 \\
40 \\
25\end{array}$ & $\begin{array}{l}1.247 \\
1.113 \\
0.904 \\
1.472 \\
1.365 \\
1.098\end{array}$ & $\begin{array}{l}1.379 \\
1.340 \\
1.375 \\
1.263 \\
1.270 \\
1.270\end{array}$ & $\begin{array}{l}0.090 \\
0.130 \\
0.293 \\
0.078 \\
0.141 \\
0.294\end{array}$ & $\begin{array}{l}1.441(0.15)^{(b)} \\
2.845(0.21) \\
5.475(0.43) \\
1.718(0.14) \\
2.410(0.21) \\
8.994(0.43)\end{array}$ & $\begin{array}{l}1.111 \\
1.121 \\
1.040 \\
1.113 \\
1.099 \\
1.040\end{array}$ \\
\hline
\end{tabular}

(a) Statistical variations are small, amounting to significant variations only in the third place.

(b) Fraction of starting neutrons.

TABLE III-III

ENERGY SPECTRA FOR THE LIPb LINER ASSEMBLY AND 50 VOT\% LITHIUM CAVITY (CASE 4, TABLE III-II), AND FOR THE CU LINER ASSEMBLY AND 25 vol\% LITHIUM CAVITY (CASE 3, TABLE III-I)

Upper Energy Limit (MeV)

$10-4$

$10-3$

$10^{-1}$

100

101

15

Total

Track Length Estimate of Flux ( $\mathrm{n} / \mathrm{m} 2 /$ shot) $10^{14}$ (Case 3) (Case 4)

\begin{tabular}{cc}
13.175 & -- \\
23.073 & 8.715 \\
50.141 & 20.48 \\
149.58 & 48.08 \\
101.60 & 23.29 \\
7.741 & 1.975 \\
1.618 & 0.1808 \\
\hline
\end{tabular}

346.85

102.71 


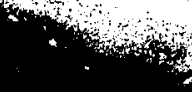

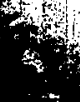




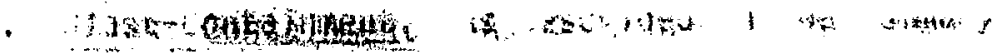

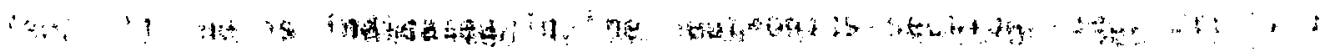

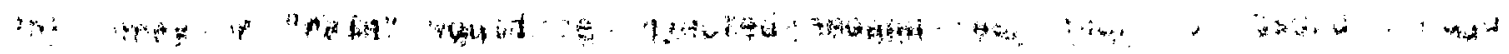

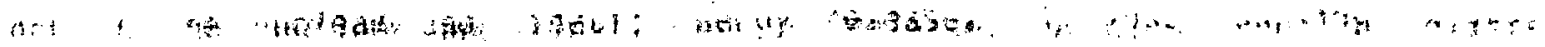

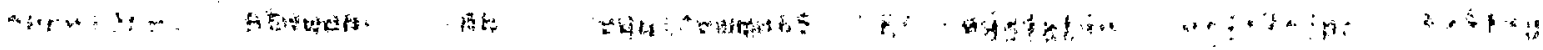

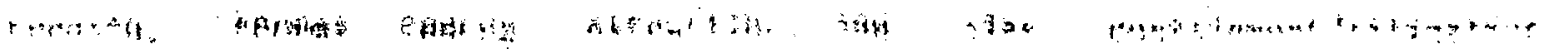

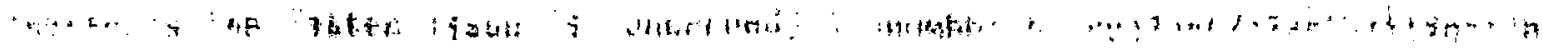

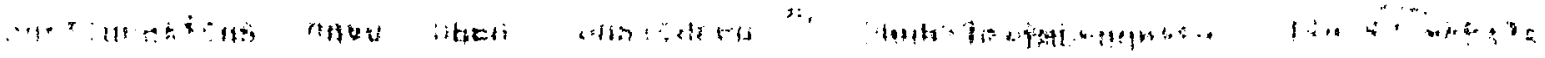

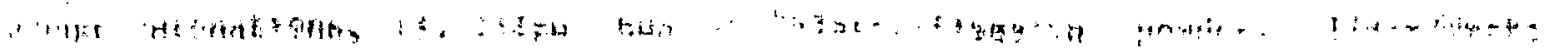

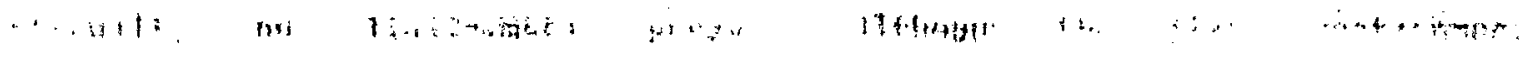

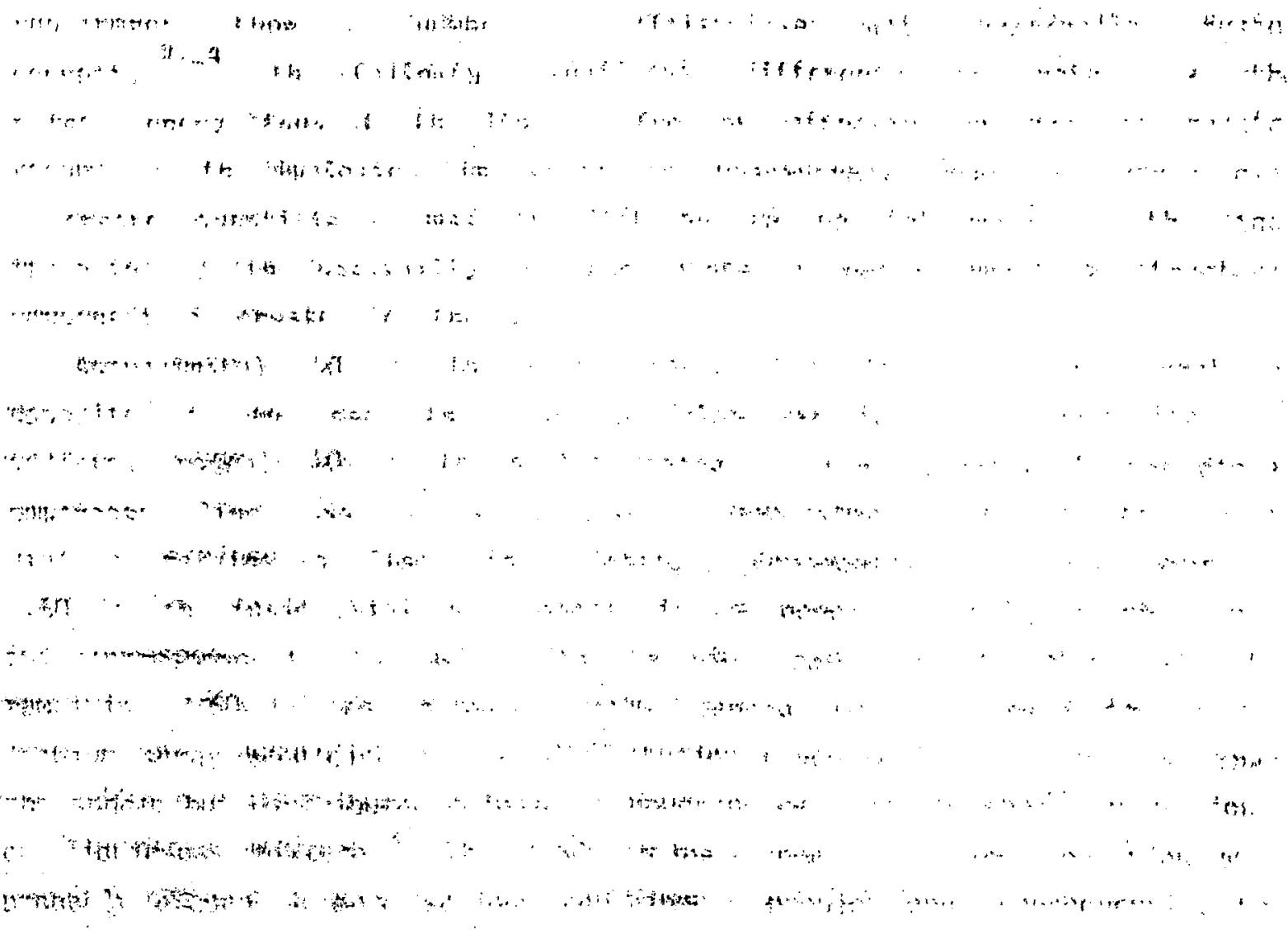

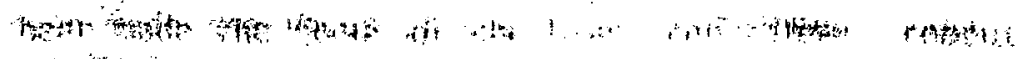

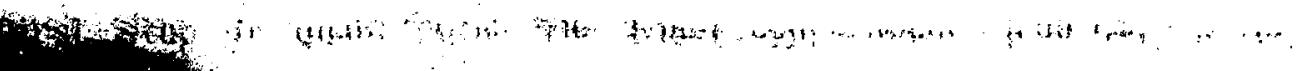

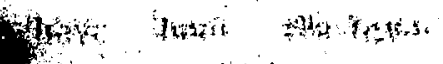

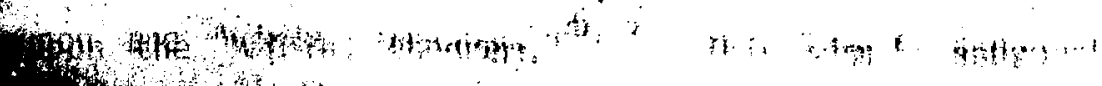

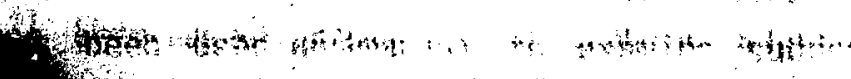

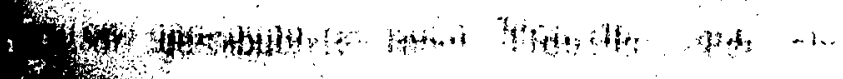

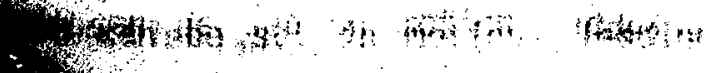


considerable pressure amplification at the containment walls (Appendix E). After a brief description of the simple containment models, this section describes the results of more detailed structural/mechanical computations.

In the simplest containment model the blast energy $W_{B}$ deposited at the initial liner position is assumed to thermalize uniformly as an ideal gas within the spherical containment vessel of radius $R$ and wall thickness $\Delta R$. The circumferenitial stress in a spherical, thin-shell pressure vesse 1 is given by

$$
\sigma_{\Theta}=W_{B} / 4 \pi R^{2} \Delta R
$$

and the corresponding strain is 38

$$
\varepsilon_{\theta}=\left[(1-v) \sigma_{\theta}+v \sigma_{r}\right] / E \text {, }
$$

where $E$ is Young's modulus, $\nu$ is Poisson's ratio, and $\sigma_{r}$ is the radially directed stress. In this discussion $\sigma_{\theta}$ is taken as tensile and $\sigma_{r}$ is compressive (both positive). These expressions predict well the results of experiments which used sinall explosive charges (few $\mathrm{kg}$ ) in relatively small spherical vessels $(R \simeq 0.5 \mathrm{~m})$ under vacuum (Appendix $E$ ). If, however, similar tests were performed either at atmospheric pressure or in the presence of blast-mitigating media (e.g., vermiculite), significant deviations from the virial-theorem approach (Eq.(IIl-27)) are observed. Fcr the range of $W_{B}, R$, and $\Delta R$ investigated, detonations carried out in air at atmospheric pressure gave shock-induced stresses, that were about four times the predictions of Eq. (III-27). The use of energy-absorbing materials reduced the stresses approximately by a factor of two. It is emphasized here that although blast-mitigation is invoked for the FLR, theoretical understanding of these processes is meager. Whether or not a blast-mitigating mechanism is available in the liquid-metal spray evoked for the FLR remains to be demonstrated. If blast-mitigation proves unfeasible with the $L i$ spray the use of "vermiculite-like" materials which are lithium bearing $\left(\mathrm{Li}_{2} \mathrm{O}, \mathrm{LiAlO}_{2}\right.$, etc.) may be required in the form of a fluidized bed. 9

of primary interest to quantifying the blast-containment problem, beyond the limits of the simple models described in Appendix E, are the time-resclved shock spectra produced at the vessel wall by the equivalent blast-energy release $W_{B}$. The computer code $P A D^{39}$ was used to compute in one- 
dimensional (spherical) Lagrangian coordinates the motion of explosive gases and the mechanical response of the spherical container. Radiation heat transfer and thermal conduction within the $\sim 1-G J$ blast created at the initial liner location were not considered, nor were asymmetry effects that may be induced by the liner-leads structure. Consistent with the sample operating points surmarized in Table II-I, blast energies $W_{B}$ in the range 0.70 to $2.26 \mathrm{GJ}$ were considered. The results of the PAD computations can be accurately scaled to other vessel sizes and energy releases according to Eqs. (III-27) and (III-28), or the results of a more detailed analytic formulation given in Appendix $E$ could be used.

For most computations $W_{B}=1.4 \mathrm{GJ}$. This energy was assumed to be deposited in a sphere with the density of solid copper $\left(8.92(10)^{3} \mathrm{~kg} / \mathrm{m}^{3}\right)$ located at the center of containment vessel. For the plirposes of this anaiysis $M$ is defined as the mass of destroyed liner and leads structure that shares the energy $W_{B}$ and contributes ultimately to the shock spectrum at the container walls. Based upon the scaling of experimental data from blasts in evacuated vessels (Appendix $E$ ), the radius $R$ of the containment sphere is estimated to be $2.6 \mathrm{~m}$ if its wall thickness $\Delta R$ is $0.15 \mathrm{~m}$ when $W_{B}=1.4 \mathrm{GJ}$. The density and Young's modulus of the containment vessel are taken to be those of 304 stainless steel $\left(\rho=7.86(10)^{3} \mathrm{~kg} / \mathrm{m}^{3}, E=160 \mathrm{GPa}\right)$. The vessel was not allowed to yield in the PAD computations. When the yield stress is exceeded in a computational result, $\Delta R$ is scaled according to Eq. (III-27) to reduce the stress to acceptable levels.

The first $P A D$ computations were made for $W_{B}=1.4 \mathrm{GJ}$ in an evacuated sphere. Two tine histories of radial and circumferential stresses ( $\sigma_{r}$ and $\sigma_{\theta}$, respectively) are shown in Fig. III-17 for blast-product masses $M$ of $25 \mathrm{~kg}$ and $200 \mathrm{~kg}$, respectively. The vessel oscillates at a frequency of $f_{v}=475 \mathrm{~Hz}$ that is independent of $\Delta R$ in accordance with the thin-shell approximation. The reverberating gas within the vessel oscillates at a frequency $f_{g}$ proportional to $\mathrm{M}^{-1 / 2}$. Since energy losses are not included in these computations, the radial stress asymptotically approaches the equilibrated pressure corresponding to a uniform distribution of the initial blast energy $H_{B}$.

The maximum circumferential or hoop stress, $\tau_{\theta}$, is plotted as a function of $M$ in Fig. III-18. This stress is nearly constant for small values of $M$, where $f_{g}>>f_{v}$. For this situation the gas pressure at the vessel wall, 
$\sigma_{r}$, oscillates and is ultimately damped to the pressure of a quiescent gas with energy $W_{B}$. Meanwhile, the vessel moves nearly as a harmonic oscillator from a condition of zero hoop stress to a maximum stress. The average hoop stress will support the pressure of a quiescent gas of energy $W_{R}$ (Eq. (III-27)). Since the shell oscillates harmonically from zero to a maximum, the

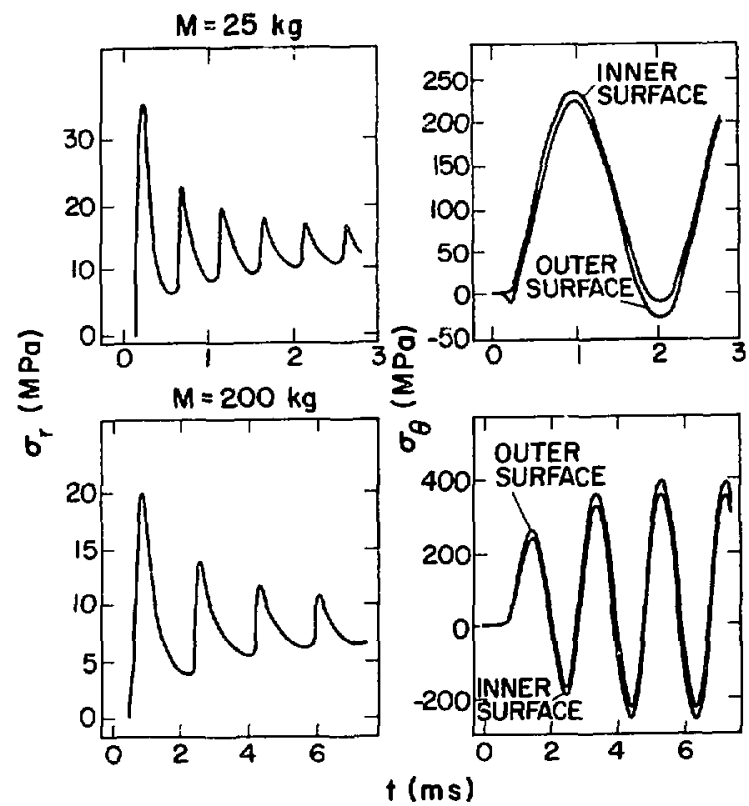

Fig. III-17. Time dependence of radial $\left(\sigma_{p}\right)$ and hoop $\left(\sigma_{\theta}\right)$ stress for a vacuum energy release of $1.4 \mathrm{GJ}$ in a spherical ve $5 \mathrm{sel}$ of $2.6-\mathrm{m}$ radius and $0.15-\mathrm{m}$ wall thickness. The mass that contains this energy is $M$. These results can be scaled to other vessel dimensions $(R, \Delta R)$ by Eq. (III-27).

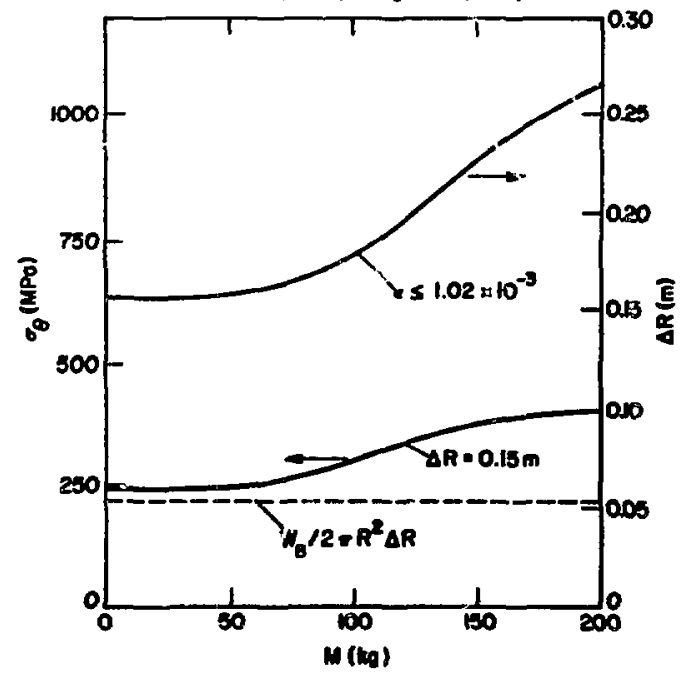

Fig. III-18. Dependence of maximum hoop stress on mass $M$ assigned to a vacuum release of $1.4 \mathrm{GJ}$ energy fur either fixed maximum strain $\varepsilon$ or a fixed vessel thickness $\Delta R$. The vessel radius is $R=2.6 \mathrm{~m}$. Dashed 1 ine indicates virial theorem prediction, Eq. (III-27). 
peak stress is approximately twice the average stress. This approximation fails when the explosive gas and shell come into resonance at $f_{g} \simeq f_{v}$, as seen for the $M=200-\mathrm{kg}$ case in Figs. (III-17) and (III-18). In this case the maximum stress is $77 \%$ higher than the value given by the above-mentioned approximation.

Based on fatigue data for stainless stee $1,{ }^{40}$ as interpreted for liner blast containment, 9 a peak strain of $\varepsilon_{\theta}=1.016 \times 10^{-3}$ appears acceptable for a 10 -yr life $\left(2.5(10)^{7}\right.$ shots for an $80 \%$ plant factor) at $800 \mathrm{~K}$. By taking $\nu=0.29, \sigma_{r}=20 \mathrm{MPa}$, and $E=160 \mathrm{GPa}$, Eq. (III-28) is used to give the maximum acceptable circumferential stress $\sigma_{\theta}$; the vessel wall thickness $\Delta R$ is then scaled to an appropriate value. For the $M=25$ and $200-\mathrm{kg}$ cases in Fig. III- 17 the $\Delta R$ values with acceptable fatigue strain are 0.16 and $0.27 \mathrm{~m}$, respectively.

The PAD code was also used to model blast containment in a liquid-gas mixture. It has been proposed that both 7 iner ${ }^{3}$ and 1 aser 32 fusion reactors be immersed in a $\mathrm{Li}$ (or $\mathrm{LiPb}$ ) spray for tritium breeding and neutron moderation. If a fast liner were immersed in a purely liquid environment, the shock wave created in the licuid would present intolerable stress amplification at the containment walls (Appendix E). On the other hand the shock may be substantially mitigated by mixing a compressible gas with the liquid. 32 The time histories of three PAD computations are shown in Fig. III-19. The blast energy $W_{B}$ is again fixed at $1.4 \mathrm{GJ}$, and the 304 stainless steel vessel parameters are, again, $R=2.6 \mathrm{~m}$ and $\Delta R=0.15 \mathrm{~m}$. A LiPb mixture of $9.4(10)^{3} \mathrm{~kg} / \mathrm{m}^{3}$ density at $\sim 870 \mathrm{~K}$ is dispersed through the vessel with a volume fraction of $f$. The volume fraction l-f is filled with helium at atmospheric pressure. The liquid is assumed to be incompressible, and the helium is regarded as an ideal gas with the heat capacity ratio $\gamma$ treated as a free parameter. Hence, the helium gas when subjected to a volumetric compression $K$ would obey the following relationships: $P / \kappa^{\gamma}=$ constant and $T / T_{0}=\kappa^{\gamma-1}$, where $T_{0}$ is the initial (pre-shot) helium temperature. An artificial viscosity term in the PAD computation produces non-adiabatic heating across the shock fronts which traverse the LiPb/He mixture.

The radial and hoop stresses as functions of time are shown in Fig. III-19 for three $\gamma-f$ couplets. These results show the sensitivity of the vessel stress response to the assumed value of liquid volume fraction $f$ and 
the $\gamma$ values of the gas phase. For $\gamma=5 / 3$ all compressive energy entering the gas-liquid mixture would ultimately heat the gas. Correspondingly, compression of the gas would be isothermal if $\gamma=1$, simulating an immediate iransfer of thermal energy to the liquid metal. The first example in Fig. III-19 $(\gamma=1.4$ and $f=0.2)$ results in a peak hoop stress of $\sigma_{\theta}=1200 \mathrm{MPa}$ for $\Delta R=0.15 \mathrm{~m}$ or a requirement that: $\Delta R$ be increased to $0.9 \mathrm{~m}$, according to Eq. (III-28), if a 10-yrfatigue constraint at $800 \mathrm{~K}$ is imposed. Unlike the vacuum containment cases (Fig. III-17), the greatest wa.11 stresses occur in a short pulse followed by smaller oscillatory stresses.
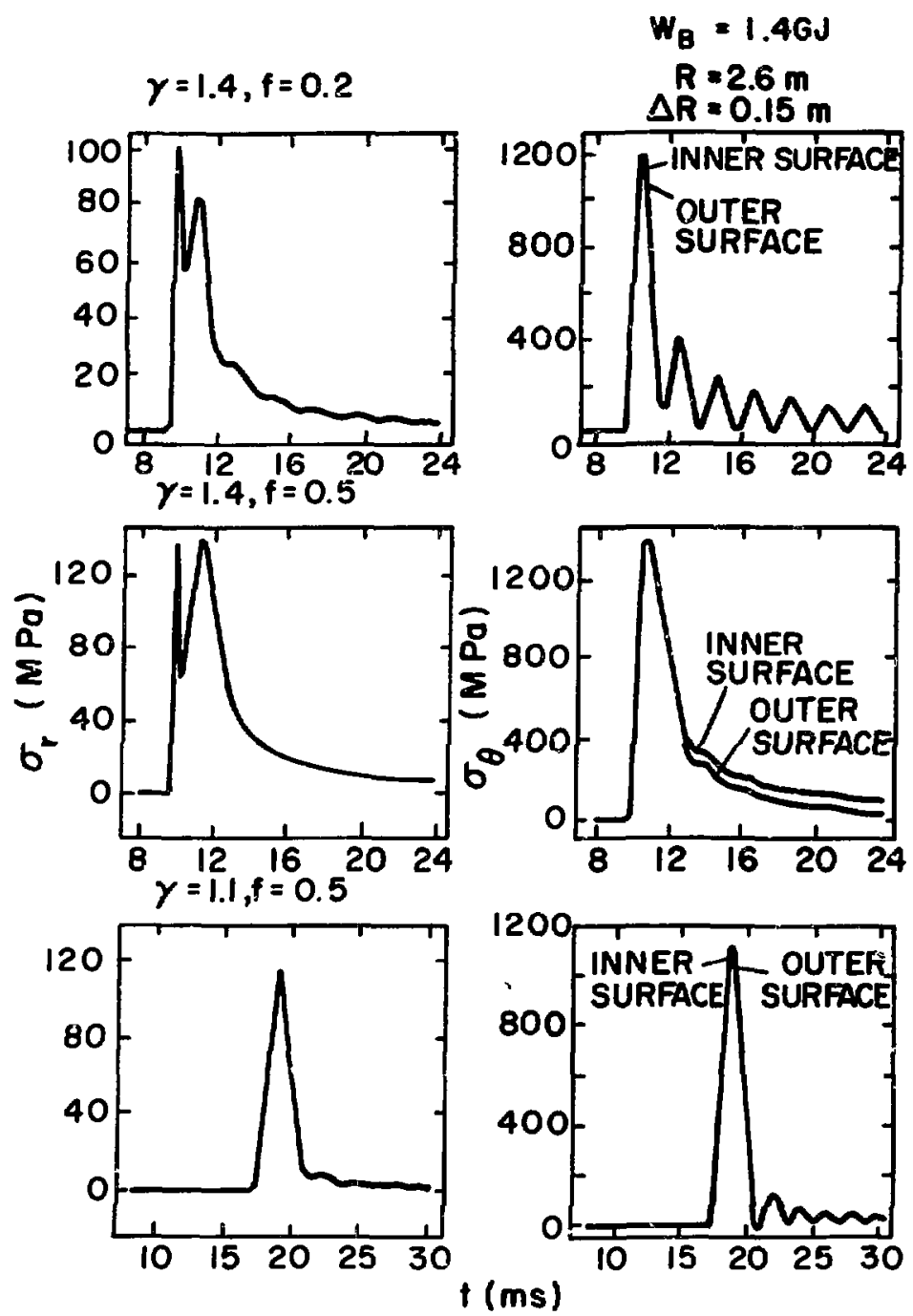

Fig. III-19. Time-dependence of radial $\left(\sigma_{n}\right)$ and hoop $\left(\sigma_{\theta}\right)$ stress for 1.4 GJ released to a $M=25-\mathrm{kg}$ mass in a gas (He) $\%$ iqquid $\left(\mathrm{LiPb}\right.$ ) ${ }^{\theta}$ mixture contained in a $R=2.6-\mathrm{m}, \quad \Delta R=0.15-\mathrm{m}$ spherical vessel that is initially pressurized to $0.1 \mathrm{MPa}$. The initial volume fraction of liquid is $f$, and $\gamma$ is the heat capacity ratio for the gas. 
For the second case given on Fig. III-19, $\gamma$ is again taken to be 1.4, but the liquid volume fraction is increased to 0.5 . The peak hoop stress increases to $1400 \mathrm{MPa}$ for $\Delta R=0.15$, or a requirement of $\Delta R=1.2 \mathrm{~m}$ results if a 10-yr fatigue constraint is imposed at $800 \mathrm{~K}$. Simple scaling argumerts indicate that the momentum impulse at the wall, $\sigma_{r} d t$, will increase roughly as $f^{1 / 2}$, but the associated increase in impulse duration makes $\sigma_{\Theta}$ relatively insensitive to $f$. This prediction is borne out by the $17 \%$ increase in $\sigma_{\Theta}$ when $f$ increases by $150 \%$.

The third example in Fig. III-19 shows the effects of a reduction in $\gamma$ from 1.4 to 1.1 while $f$ is held at 0.5 . This model simulates the rapid transfer of shock energy to the liquid metal (i.e., the $\gamma \rightarrow 1$ limit). Since the temperature rise in the helium is smalier for a given compression when $\gamma$ is decreased from 1.4 to 1.1 the liquid-gas mixture is more easily compressed. A somewhat smaller momentum is transferred to the liquid metal, and a reduced stress occurs at the vessel wall; this hoop stress equals $1100 \mathrm{MPa}$, and corresponds to $\Delta R=0.9 \mathrm{~m}$ for an acceptable stress.

A11 cases shown in Fig. III-19 exhibit a sharp stress pulse that lasts about $3 \mathrm{~ms}$. This intense, initial pulse could be reduced in peak intensity and spread out in time by a blast-attenuating structure attached to the inside wall of the containment vessel as indicated in Fig. II-1. For example, the shock velocity is $\sim 100 \mathrm{~m} / \mathrm{s}$ and the particle velocity is $\sim 50 \mathrm{~m} / \mathrm{s}$ at the time the shock impacts the structural wall. By placing rib-like structures on the inner walls that are $0.3 \mathrm{~m}$ high and filling $50 \%$ of the local volume, the duration of impact may be increased by a factor of n, 2, which in turn would cause the maximum $\sigma_{\Theta}$ to be reduced by a comparable amount. Therefore, blast atcenuators may significantly reduce the overall structural requirements placed on the containment vessel. The concept of physical shock attenuators, however, must be examined by more detailed analysis.

The foregoing examination of blast containment is based on a number of simplifying assumptions. Present theoretical predictions and extrapolation of the existing data base should be treated as imprecise until experimental tests are made for much nigher blast energies. The general scale of blast requirements has been quantified, however, and appears to be technologicaliy feasible. Generally, 2.5-to 3.0-m-radius containment vessels with 0.3-to 0.5-m-thick walls appear adequate to contain the $\sim 1.5 \mathrm{GJ}$ of thermal energy expected to be released every $\sim 10 \mathrm{~s}$ by the FLR; these dimensions appear 
adequate for a 10-yr fatigue life at $800 \mathrm{~K}$ for stainless steel. By proper vessel desic.l (physical shock attenuators) and selection of blast-mitigating media, the uncertainties associated with the models used to generate these results can be reduced; based on present knowledge it is doubtful that either $R$ or $\Delta R$ will be decreased for the low-yield design point given on Table II-I. The need to build more conservatism into the vessel design will become more apparent when the effects of long-term radiation damage and the realities of actual engineering structures (penetrations, weldments, etc.) are examined.

The neutronics computations given in SeC. III.B.5 are hased on a lithium-helium mixture inside the containment vessel rather than a LiPb-He system. Blast computations have not been made for the $L i$ case, but results from such an exercise would surely fall between the vacuum versus LiPb extremes considered above. Although the lithium density is much less than that of the $\mathrm{LiPb}\left(475 \mathrm{~kg} / \mathrm{m}^{3}\right.$ versus $\left.9400 \mathrm{~kg} / \mathrm{m}^{3}\right)$, the stress expected for the Li-He mixture would not be correspondingly close to the vacuum case, since a shock wave is established even in a light-weight fluid. As noted previously, blasts in air may produce four times as much stress as vacuum contained blasts. For simplicity it is assumed that a 1.4-GJ blast in purz lithium and helium at atmospheric pressures and volume fraction $f=0.4$ requires a wall $0.3 \mathrm{~m}$ thick with a ribbed inner wall. It is also assumed that the scaling of Eq. (III-27) applies for other energies.

7. Heat Transfer. As seen from Table III-I the following distribution of thermal energy release to the blast cavity is expected for the low-yield case: enhanced fusion yield $=3920 \mathrm{MJ}$; liner kinetic energy $=336 \mathrm{MJ}$; leads losses $=37 \mathrm{MJ}$; plasma preparation $=7.5 \mathrm{MJ}$, which gives a total thermal release of $4.3 \mathrm{GJ}$ to the blast cavity. Figure III-20 gives the radial distribution of the nuclear energy density (neutrons plus gamma rays) deposited into a lithium spray of three possible volume fractions (25, 40, and 50 vol\%), as determined by the MCNP Monte Carlo calculations described in Sec. III.B.5. Approximately $1.5 \mathrm{GJ}$ would be deposited in or near the initial liner volume (Sec. III.B.6), which amounts to $5.9(10)^{10} \mathrm{~J} / \mathrm{m}^{3}$ in the region $r=0$ to $0.2 \mathrm{~m}$ indicated on Fig. III-20.

Neglecting the heat of fusion associated with the leads structure, $4.3 \mathrm{GJ}$ is capable of uniformly increasing the 50 vol\% lithium in the 2.6-m-radius vesse 1 by $59 \mathrm{~K}$. As indicated in Sec. III.B.6, however, the means by which this highly anisotropic energy-density distribution nondestructively relaxes 


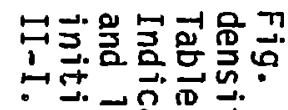

巳一。

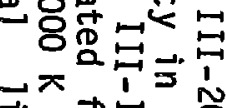

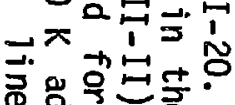

ญ ․ㅜㄱ

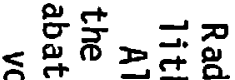
무요 득옥올 吉思票的。

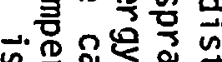

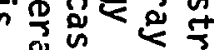
产兽弯

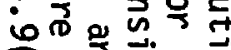

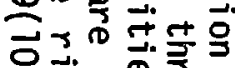

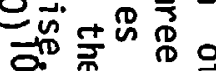
号本里 옳

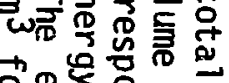
옥을

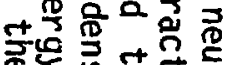
두웡

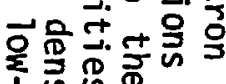

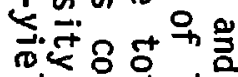

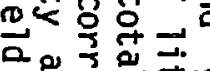

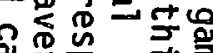

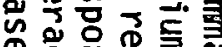
吕合自 ¿․ㅡ응

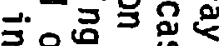
亩+5希 TOTAL ENERGY DENSITY $\left(\mathrm{J} / \mathrm{m}^{3}\right)$

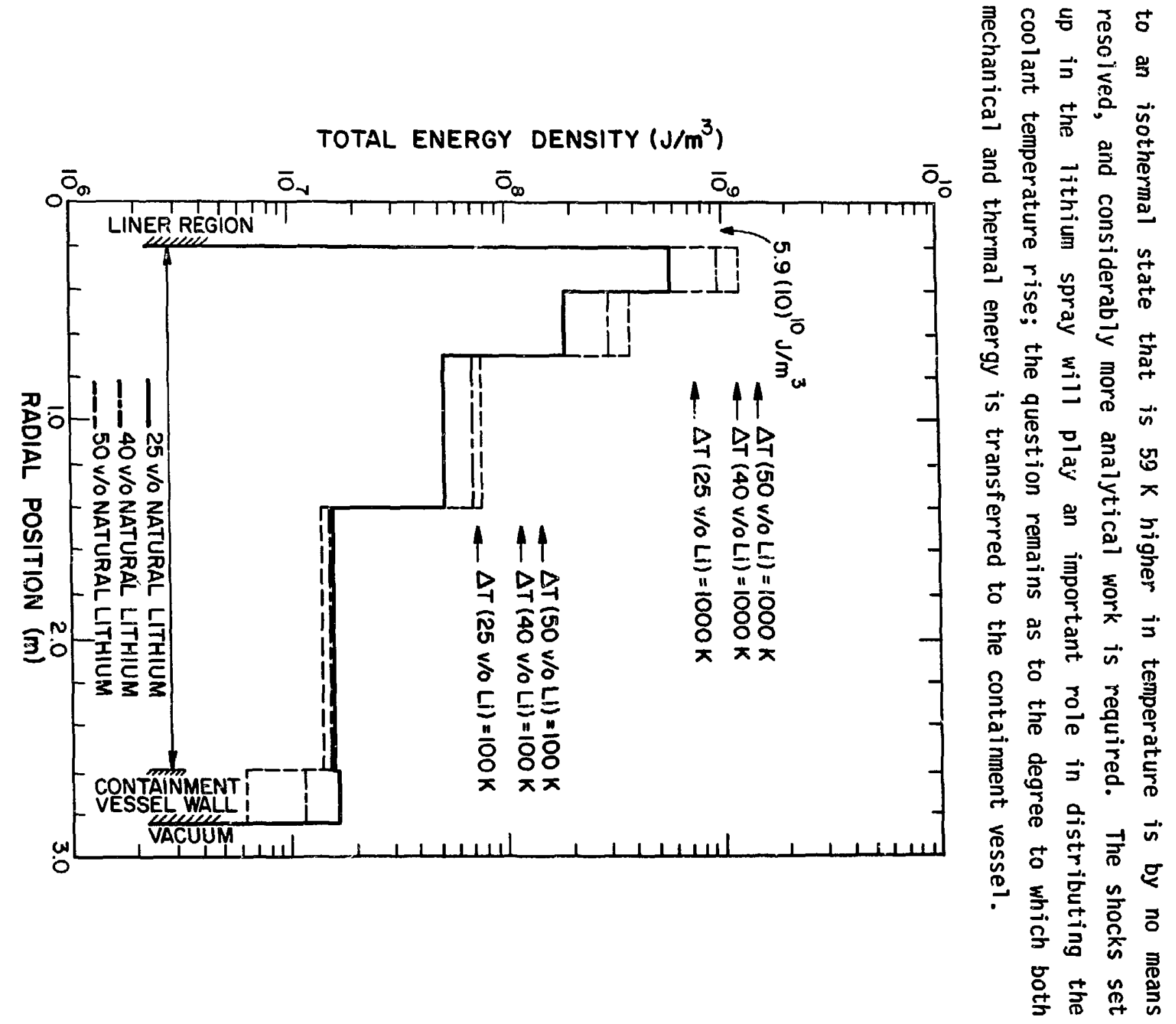

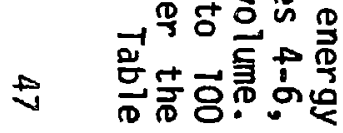


Although the complex processes which govern the pulsed heat transfer within the FLR blast cavity have not been subjected to quantitative analysis, a qualitative description is given below, which could serve as the basis of analytic modeling. The exponentially decreasing neutron and gamma-ray heating rate in the lithium-spray blanket causes a large $(\sim 300 \mathrm{~K})$ radial temperature gradient in the dispersed lithium coolant spray. This large thermal gradient is further aggravated by the high heat flux at the lithium surface near the vaporized liner. A portion of the lithium in the region adjacent to the liner could also be vaporized to a depth of about $0.20 \mathrm{~m}$ from this heat flux. This hot "bubble" of vaporized $L i$ and liner materials could expand radially outward. The cooler lithium droplets would be accelerated radially by the ensuing shock wave, which also compresses the interstitial gas until complete contact with the containment vesse 1 wall occurs. As the impact pressure loading is absorbed in the vessel, it will rebound, throwing the lithium toward the center of the cavity. The compressed gas would expand as the pressure is relieved and would tend to accelerate the liquid in all directions, although it is expected that dispersion as droplets towards the cavity center would principally occur. The liquid lithium would traverse the vaporized material at the center, mixing and condensing the vapor on the droplets. The coolest liquid adjacent to the vessel wall may not participate fully in this stage of mixing, but will tend to fall more rapidly into the sump (Fig. II-1) than the less derise central region. Further mixing will take place in the shear layer between this cooler and the hotter (perhaps a still partially vaporized) material at the center. With proper design of spray nozzles, it should be possible to tailor the lithium volume fraction as a function of vessel radius in order to optimize the efficiency of energy absorption and subsequent mixing. A void space adjacent to the liner would give a larger heat transfer area at this point and may lead to reduced heat fluxes, thereby reducing the vapor fraction. A low lithium fraction near the vessel wall would increase the volume of compressed gas and the "rebound" potential from the wall. The violence of these processes should lead to very complete mixing on a short time scale. If necessary to complete condensation of the vaporized lithium at the center, a small "afterspray" could be directed at this area commencing at the end of the burn. An "afterspray" would also serve to protect the liner insertion mechanism and vessel head from the hot lithium "bubble," if it should form. 
An analytic resolution of these processes would require computer codes not unlike computer codes used to model fission reactor melt-down accidents. In addition to understanding the complex interaction between heat-transfer, shock, and irreversible blast-mitigation processes, the actual heat deposited onto the blast vessel wails remains an issue that should be examined in more detail.

For the purpose of this study it is assumed that the dynamics of the lithium spray and the time of each liner shot can be correlated to an extent where all thermal energy releases are absorbed by a vessel inventory of 50 vol\% lithium ( $74 \mathrm{~m}^{3}$ or 18 tonne) spray coolant. This lithium, therefore, is heated $58 \mathrm{~K}$ and falls into a reservoir or sump (15, Fig. II-1) of lithium with a similar temperature $(500 \mathrm{~K})$. Lithium is circulated by a centrifugal pump (16, Fig. II-1) to an intermediate sodium/lithium heat exchanger (17, Fig. II-1), and eventually to a storage tank (18, Fig. II-1) prior to re-injection into the blast cavity. Approximately 1.75 tonne/s of lithium coolant flow would be required to remove the $4.3 \mathrm{GJ}$ of thermal energy deposited once every $10 \mathrm{~s}$. The dynamics of the energy transfer within the

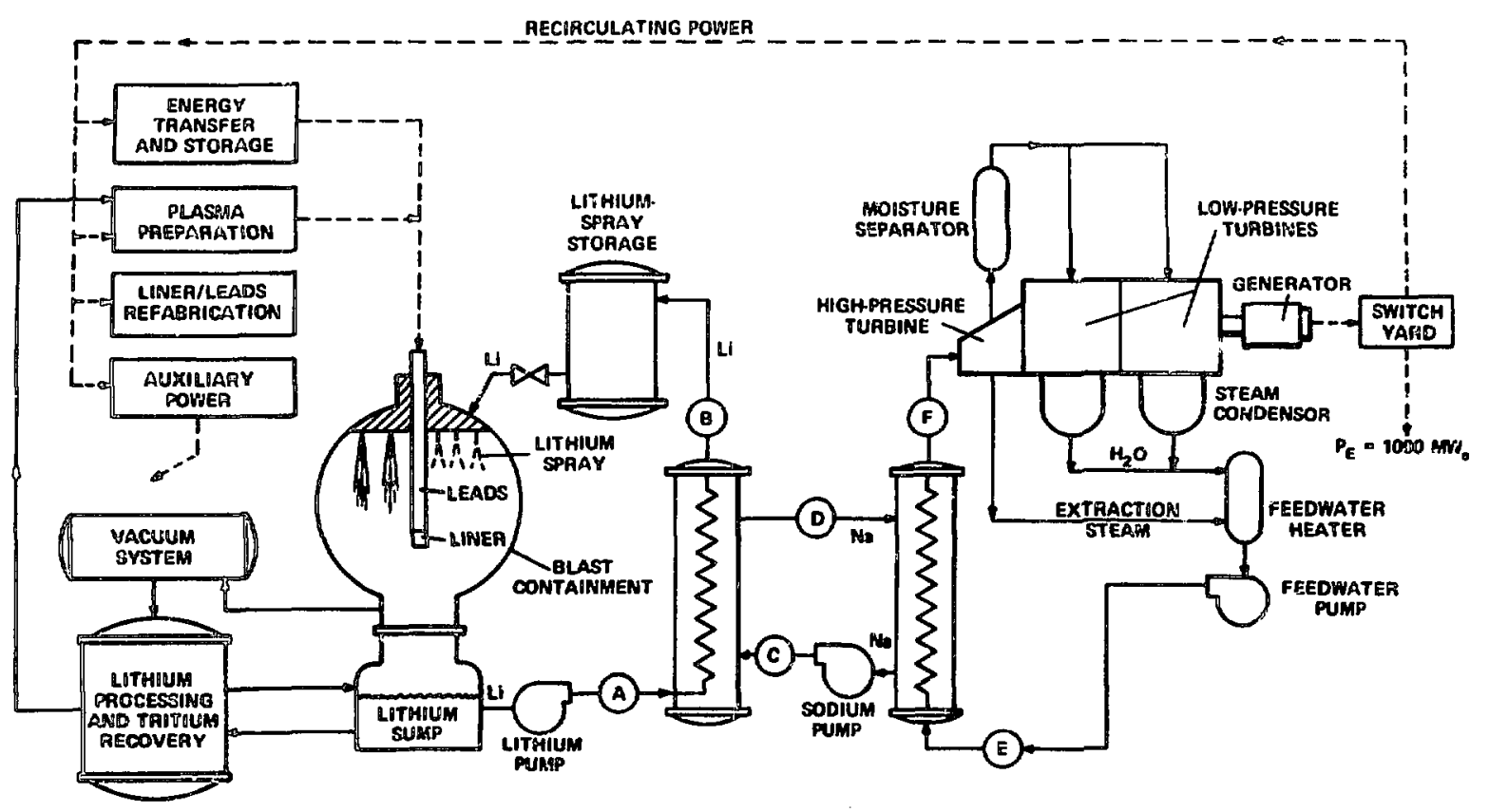

Fig. III-21. Schematic diagram of major coolant flows for the FLR system using the lithium-spray primary coolant. Table III-VI gives the stream conditions. 
blast containment is assumed to be sufficientiy rapid to preclude a significant thermal excursion at the walls of the blast containment. A schematic diagram of the FLR coolant and heat-extraction systems is shown in Fig. III-21, and Sec. III.D.1 gives a brief summary of key engineering parameters based thereon.

\section{TABLE III-IV}

\section{SUMMARY OF STREAM CONDITIONS FOR FLR SYSTEM (FIG. III-21)}

\begin{tabular}{|c|c|c|c|c|}
\hline Stream & $\begin{array}{l}\text { Temperature } \\
\left({ }^{\circ} \mathrm{C}\right) \\
\end{array}$ & $\begin{array}{l}\text { Flow Rate } \\
(\mathrm{kg} / \mathrm{s})\end{array}$ & $\begin{array}{c}\text { Pressure } \\
\text { (MPa) } \\
\end{array}$ & $\begin{array}{c}\text { Number of } \\
\text { Blast Cavities } \\
\end{array}$ \\
\hline A & 500 & 1750 & 0.11 & 1 \\
\hline B & 440 & 1750 & 0.0 & 1 \\
\hline$c$ & 288 & 4700 & 0.68 & 2 \\
\hline$D$ & 435 & 4700 & 0.68 & 2 \\
\hline$E$ & 219 & 930 & 6.8 & 4 \\
\hline$F$ & 286 & 930 & 6.8 & 4 \\
\hline
\end{tabular}

C. Costing Mode 1

Economic guidelines developed by Battelle Pacific Northwest Laboratories 41,42 are used for the costing framework. The difficulties in comparing various cost models has led to the development of this common costing procedure and should provide the needed uniformity in assessing different concepts. The costing guidelines describe uniform accounting categories and procedures, although a uniform $\cos i$ data base is yet to be adopted. A cost data base, therefore, has been generated by LASL to provide an inierim optimization tcol and to facilitate comparisons. It is emphasized that absolute cost values are intended only for the intercomparison of reactor designs and are not intended for absolute comparisons with existing energy technologies on the basis of present costs. ${ }^{43}$ The cost accounting procedure, costing guidelines, and the cost data base are given in Appendix F. Figure III-22 gives a schematic diagram of the LASL interactive costing procedure which interfaces directly with the reactor design code. 


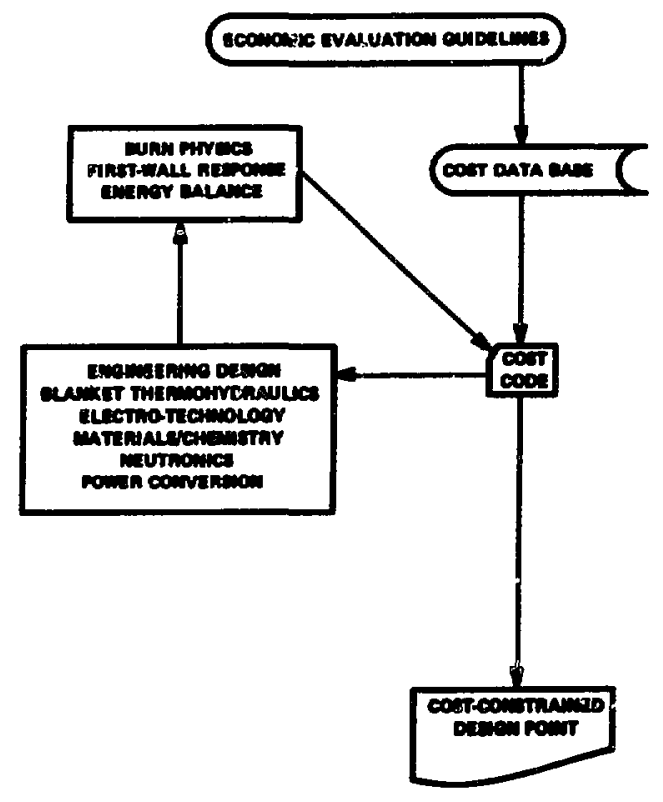

Fig. III-22. Schematic diagram of LASL Interactive Costing Program used to determine FLR power cost.

The total capital cost of the plant is composed of direct, indirect, and time-related (escalation and interest) costs. Direct costs are quoted in 1978 prices, result from the purchase of materials, equipment, and labor, and take into account allowances for spare parts and contingencies. Indirect costs, taken as a percentage of the direct costs, result from support activities necessary to complete the project and are divided into three major accounts: 15\% for construction facilities, equipment, and services; 15\% for engineering and construction management services; and $5 \%$ for taxes, insurance, staff training, and plant startup. Escalation and interest are computed as a percentage of the direct plus indirect costs assuming a 10-yr construction period. Aggregrate percentages of $33.8 \%$ and $64.4 \%,{ }^{43}$ respectively, result in an escalation rate of $5 \%$ and interest rate of $10 \%$. Having determined the total capital cost $c_{D}(\$ / \mathrm{kWe})$, the power cost $c_{p}$ (mills/kWeh) is computed on the basis of a 15\% return on capital investment, an added $2 \%$ of the total capital cost for operating expenses, and a plant factor of 0.85 . 
The FLR leads and liner replacement cost represents a unique operating cost for this concept that is not unlike a fuel cost. As noted in Sec. III.B.4 and Appendix C, if conductor recycie costs and insulator fabrication costs can be kept below $0.01 \$ / \mathrm{kg}$ and $0.10 \$ / \mathrm{kg}$, respectively, the leads/liner cost should amount to no more than $\sim 20 \%$ of the plant revenue for the low-yield design point sumarized on Table II-I. The level of the liner/leads design is not adequate to permit the detailed costing of the associated refabrication plant, and the costing of the leads/liner assembly, therefore, is based on the assumed materials and handling cost using the optimized leads geometry described in Appendix C; specifically, the leads cost is treated as an increment to the plant operating cost.

\section{Design Point}

Sections III.B.T through III.B.7 and the associated Appendix material summarized the scoping calculations that have been used to assess the major areas of technology and engineering anticipated for the FLR concept. These computations were guided primarily by the physics optimization and design point that emerged from the analyses discussed in Sec. III.A. As noted previously, the FLR concept portends a relatively simple high-power-density system, aside from the problems associated with a relatively rapid and large energy transfer and storage requirement. Because of the non-conventional physics and technological requirements identified for the FLR approach, much of the foregoing analysis had to be developed specifically for this study; the range of operating points projected to date has little basis in experiment. For these reasons the specific design point embodied in Table II-I and the extension of this design point into a more detailed estimate of engineering and cost parameters should be viewed as indicative; the engineering design point summarized in this section must be viewed as the best guess available ai this time on the basis of the complex analytic task and absence of a relevant experimental base.

Central to the development of a reactor-embodiment for the FLR is the blast-confinement and primary heat exchange systems. A liquid-lithium spray was adopted and subjected to engineering evaluation in Secs. III.B.5-III.B.7. Other corifinement/heat-transfer schemes have also been considered. Specifically, three FLR confinement schemes have evolved and are depicted in Fig. III-23. First, the liquid-metal LiPb/gas-bubble (He) concept was 


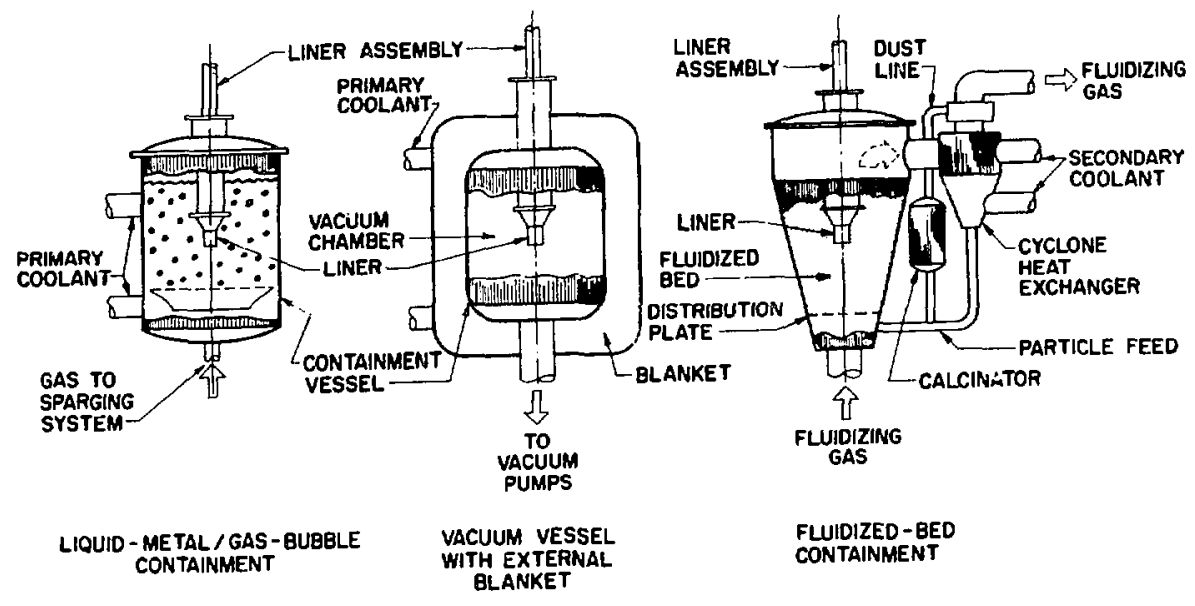

Fig. III-23. Schematic diagrams of several blast-containment and primary coolant schemes considered for the FLR.

developed, wherein a liner/leads assembly would be plunged into the two-phase coolant and detonated in much the same way proposed for certain laser/pellet fusion schemes. 32,33 Unacceptably high pressure amplification at the container wall by shock reflection was computed (Ref. 9, Appendix E) when the He bubbles occupy a substantially smaller volume fraction than the liquid metal; therefore, liquid-inetal schemes employing a small gas fraction were rejected. The favorable scaling of containment vessel size with blast energy, as predicted by the virial theorem, 36,37 and the agreement that this theory gave with experimental data 35 led to the consideration of implosions detonated in vacuo; the vacuum chamber would be surrounded by a neutronattenuating, tritium-breeding blanket. Although this concept has not been rejected, the potential problem of rapid insertion of liner assemblies, and the use of high voltage in vacuo, and the potential of damage to the vacuum wall by radiation and massive, energetic debris has resulted in consideration being given to a concept wherein the liner/leads assembly would be suspended in a fluidized bed of lithium-bearing particles ${ }^{10}$ (oxide or aluminate). Operating at $30 \%-50 \%$ of solid density, the bed would absorb nearly all 
neutrons, the particles would be pul:erized under the action of the post-implosion shock, would breed tritium, and with the carrier gas (He) would serve as the primary heat-exchange fluid. After the fluidized bed "recovers" from a given shot, the fine, pulverized particles (and thermal energy) would be removed from the system by the carrier gas, cooled, cyclone-separated from the carrier gas, resintered, and cycled back to the fluidized bed. The pulverizing action of the post-implosion shock would also release bred tritium from the bed particles, and the released tritium could easily be recovered from the He carrier gas by oxidation. Large sintered particles generated within the fluidized bed (typically at the container walls) as well as large pieces of liner debris attenuated by the fluidized particles, would fall out and be collected for reprocessing.

A fourth containment scheme, adopted by this study, would be similar to the 1iquid-metal/gas system noted above, but instead would inject the lithium (perhaps with lead) as a spray or "rain" into the liner cavity. This concept is similar to a scheme proposed by Burke 12,44 for electron-beam fusion, and has been analyzed in Sec. III.B.6. Both the lithium-spray and the fluidizedbed schemes are considered as viable contenders for the tasks of blast confinement and primary heat transfer, although only the former is discussed here.

On the basis of the low-yield physics design point given in Table II-I and the scoping study of key engineering systems, the point design parameters given in Table III- $V$ have been developed, following the guidelines given in Ref. 45. This engineering design point was evaluated using the costing model described in Sec. III.C. Table III-VI sumarizes the capital cost according to a standardized costing account, ${ }^{41-43}$ and Table III-VII summarizes the bottom-line unit operating and power costs. It is noted that the leads/liner costs have been estimated according to a separate optimization procedure described in Sec. III.B.4 and Appendix C. At the estimated $10.09 \mathrm{mills} / \mathrm{kWeh}$ ( $3.56 \$ /$ shot) (based upon the melting leads option (Appendix $\mathrm{C}$ ), $0.10 \$ / \mathrm{kg}$ for insulator costs, and $0.01 \$ / \mathrm{kg}$ for conductor costs) the leads/liner costs amount to $16 \%$ of the total power costs. If an upper limit of $30 \%$ is established for the percent of total power cost to be assigned to leads/liner expenditures, a maximum of $6.70 \$ /$ shot is allowed. 
TABLE III-Y

SUMMARY OF KEY FLR DESIGN PARAMETERS FOR THE LOW-YIELD FLR (TABLE II-I) VALUES GIVEN PERTAIN TO A SINGLE BLAST CAVITY OF WHICH 8 ARE REQUIRED TO SUPPLY 1000 MWe(net)

?. POWER OUTPUT

$\begin{array}{lc}\text { UNIT } & \text { VALUE } \\ \text { MWt } & 425 \\ \text { MNe } & 0.0 \\ \text { MNt/m } & 4.8(a) \\ \text { MWe } & 170(b) \\ \text { MWe } & 129(c)\end{array}$

2. REACTOR COOLANT SYSTEM

*2. 1 B Tanket Coolant Type

*2.2 Blanket Out let Temperature (Hot Leg)

*2.3 Blanket Inlet Temperature (Cold Leg)

*2.4 Blanket Out let Pressure

*2.5 Blanket Inlet Pressure

*2.6 Blanket Coolant Flow Rate

2.7 Blanket Coolant Pipe Material

${ }^{0} \mathrm{C}$

${ }^{0} \mathrm{C}$

$\mathrm{MPa}$

$\mathrm{MPa}$

$\mathrm{kg} / \mathrm{s}$

$\operatorname{Li}($ liquid)(d)

500 (d)

442

2.7 Blanket Coolant Pipe Material

*2.8 First Wall Coolant Type

*2.9 First Wall Outlet Temperature

*2. 10 First Wall Inlet Temperature

*2.11 First Wall Outlet Pressure

*2.12 First Wall Inlet Pressure

*2.13 First Wall Coolant Flow Rate

2.14 Total Number of Blanket Coolant Loops

2.15 Type of Blanket Coolant Circulator

2. 16 Power Input to Each Circulator

*2.17 Peak Blanket Temperature in Case of Loss of Coolant Flow

$\begin{array}{lc} & \text { Steel } \\ & (2.25 \mathrm{Cr}-1.0 \mathrm{Mo}) \\ & \mathrm{NA} \\ { }^{\circ} \mathrm{C} & \mathrm{NA} \\ { }^{\circ} \mathrm{C} & \mathrm{NA} \\ \mathrm{MPa} & \mathrm{NA}(\mathrm{g}) \\ \mathrm{MPa} & \mathrm{NA} \\ \mathrm{kg} / \mathrm{s} & \mathrm{NA} \\ & \mathrm{l} \text { (per two } \\ & \text { blast cavities) } \\ & \text { centrifugal } \\ \mathrm{MWe} & \text { 1.6 (per two blast } \\ & \text { cavities)(h) } \\ { }^{\circ} \mathrm{C} & \text { (i) }\end{array}$

*Information on items with asterisk to be supplied by LASL designers, whereas undesignated ${ }_{\text {Corporation. }}$ items represent first estimates to be refined by Bechte 1 
TABLE III-V Cont'd.

UNIT

\section{INTERMEDIATE COOLANT SYSTEM}

3.1 Coolant Type

3.2 IHX Out let Temperature (Hot Leg)

3.3 IHX In let Temperature (Cold Leg)

3.4 IHX Outlet Pressure

3.5 IHX Inlet Pressure

3.6 Coolant Flow Rate

3.7 Coolant Pipe Material

3.8 Total Nuñber of Coolant Loops

3.9 Type of Coolant Circulator

3.10 Power Input to each Circulator

3.11 Number of IHX Per Loop

3.12 IHX Material-She 11/Tube

4. STEAM GENERATION SYSTEM UNIT

4.1 Steam Out let Temperature

4.2 Steam Outlet Pressure

4.3 Steam Flow Rate

4.4 Feedwater Temperature

4.5 Number of Steam Generators per Loop

4.6 Number of Modules per SG

4.7 SG Materials, Shell/Tube
${ }^{\circ} \mathrm{C}$

${ }^{\circ} \mathrm{C}$

$\mathrm{MPa}$

$\mathrm{MPa}$

$\mathrm{kg} / \mathrm{s}$

MWe

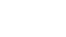


TABLE II I-V Cont'd.

*5.6 Coolant Inlet Pressure
MPa
0.2
$\mathrm{kg} / \mathrm{s}$
75.6

*5.7 Coolant Flow Rate

6. REACTOR AUXILIARY SYSTEMS

UNIT

VALUE

6.1 Vacuum Pumping System

(1)

*Plasma Chamber Pressure

torr

*Plasma Chamber Volume

$m^{3}$

1

* Number of Pumps

*Capacity of Each Pump

4

torr-1iter/s $17.5(10)^{3}$

6.2 Magnet Cooling System

*Cooling Load

Wt

NA

6.3 Plasma Heating System

*Cooling Load

Wt

NA

6.4 Tritium Processing and Recovery System

*Total Tritium Inventory

$\mathrm{kg}$

$18(m)$

7. REACTOR COMPONENTS

7.1 Blanket/First Wall

*Material

Stee $1(2.25 \mathrm{Cr}-1.0 \mathrm{Mo})$

* Number of Modules

NA

*Weight of Each Module

Tonnes

NA

*Weight of Largest Single Component Tonnes

$200(n)$

*Dimensions of Largest Component mxmxm

7.2 Shielding(concrete biological shield)

$5.2-\mathrm{m}(\mathrm{id}) \times 0.3 \mathrm{~m}(\mathrm{wa}) 1)$

*Material

(o)

* Number of Modules

* Weight of Each Module

Tonnes

* Weight of Largest Single Component Tonnes

*Dimensions of Largest Component mxmxm

Concrete/stee $]$

NA

NA

NA

NA

7.3 Magnet

*Coil Forces Transmitted to Building

Newton

NA

7.4 Reactor Assembiy

*Total Weight of Reactor Assembly Tonnes

$200(p)$ 
TABLE III-V Cont'd.
8. BUILDINGS
UNIT
VALUE
8.1 Containment Building
*Minimum Wall Thickness for Shielding
m
$(0, p)$
*Internal Pressure, Normal/Accident
$\mathrm{MPa}$
1
*Containment Atmosphere
$\mathrm{atm} / \mathrm{atm}$
Argon
8.2 Electrical Energy Storage Building
*Wall Thickness for Shielding
m
0.0
*Internal Pressure, Normal/Accident
$\mathrm{MPa}$
$\mathrm{atm} / \mathrm{atm}$
*Safety Related or Not
Yes/No
yes $(q)$

9. ELECTRICAL POWER REQUIREMENTS(for 8 units)

*Cold Stari Power from Grid

MWe vs

NA( $r)$

Auxiliary Power Requirement(Normal Operation)

MWe

*Electrical Energy Storage

* Magnet Power Supply (Other than energy storage)

Blanket Circulators

First Wall Coolant Circulators

Shield Coolant Circulators

*Refrigeration System

*Vacuum System

*Plasma Heating System

*Miscellaneous Reactor Plant Auxiliaries

Feed Pump System

$312(r)$

NA

6.4

NA

$1.0(s)$

nil $(t)$

$0.2(u)$

$8.0(v)$

14. (w)

TBD

(Bechte1 Corp)

Condensing System

TBD

(Bechte 1 Corp)

Heat Rejection System

TBD

(Bechte] Corp)

TBD

Misc. BOP Auxiliaries

(Bechtel Corp)

10. REACTOR MAINTENANCE

10.1 Blanket/First Wall Replacement

Tonnes/yr

$20(x)$

10.2 Radioactive Material Storage

$\mathrm{Yr} / \mathrm{m}^{3}$

UNK

Requirement; Years/Volume

UNK

10.3 Description and Sketches of Replacement Concept 
TABLE III-V Cont'd.

11. REACTOR ASSEMBLY

*Detailed Dimensional Drawings of Reactor Assembly (Re:Sec.II,III.B)

(a) Based on volume enclosed and included by blast-confinement vessel. Average power density within initial liner volume is $17.1 \mathrm{GWt} / \mathrm{m}^{3}$.

(b) Based on thermal conversion efficiency $n_{\mathrm{TH}}=0.40$.

(c) Based on a computed recirculating power fraction $\varepsilon=0.25$ or a net plant efficiency of $n_{p}=n_{T H}(1-\varepsilon)=0.30$. In order to generate 21000 MWe (ret) 8 blast cavities would be required, each cavity being energized by the same ETS system.

(d) Liquid-1ithium spray injected at 40-50 vol\% into blast cavity and around liner. For a 2.6-m-radius sphere and the energy released each shot, an average $58 \mathrm{~K}$ temperature rise would occur within the lithium spray. The flow rate is adjusted to give one vessel volume $\left(\sim 70 \mathrm{~m}^{3}\right)$ recycled each pulse period (10 s).

(e) Based on a 20-m lithium head. This value actually represents the pressure differential across the lithium pump, the pressure at the spray-coolant inlet per se being zero.

(f) The lithium spray serves as the blanket, which is injected into the blast cavity under vacuum.

(g) First wall is considered here to be the blast cavity wall, and is both shielded and cooled by the primary lithium-spray coolant. For this reason items 2.8-2.13 are not applicable (NA).

(h) Based on $55 \%$ efficient pump, 0.11-MPa head, and a $7-\mathrm{m}^{3} / \mathrm{s}$ average flow rate (half a cavity volume in $10 \mathrm{~s}$ ).

(i) The averaged surface energy density if the liner and fusion (alpha-particle) energy were completely unattenuated (i.e., spray blanket did not form) amounts to $13.4 \mathrm{MJ} / \mathrm{m}^{2}$. This energy is capable of raising $3.4 \mathrm{~mm}$ of the steel vessel to the melting point.

(j) Both the sodium intermediate loop and the steam cycle for the FLR are scaled from the results reported by J. C. Scarborough ("Competitive Capital Costs for the Prototype Large Breeder Reactor," ANS Winter Meeting, San Francisco, CA, November 27-December 2, 1977, data reported in NUS Corp. publication). The GE/Bechtel Saturated Steam system was used. Each of four 726-MWt intermediate loops gave $T($ IN)/T(OUT) = $280^{\circ} \mathrm{C} / 435^{\circ} \mathrm{C}$; $0.56 \mathrm{MPa}$ pump head at $4.33 \mathrm{~m}^{3} / \mathrm{s}\left(4.03(10)^{3} \mathrm{~kg} / \mathrm{s}\right)$ flow rate, head power $=2.43$ MWe; pump power $=5.6 \mathrm{MWe} ; 1389$ MWe s/kg. Two FLR cavities would provide $850 \mathrm{MWt}$, so the GE/Bechtel loops have been scaled by $850 / 726=1.17$. Four such units would provide $3400 \mathrm{MWt}$, which gives approximately the design goal of 1000 MWe(net). Two 1700 MWt steam generators are required, each driven by two such sodium loops, which are in turn driven by 4 blast cavities. 
TAB!E III-V Cont ' $d$.

Footnotes Cont'd.

(k) The shielding requirements for the FLR will not differ significantly from those envisaged for ex-pressure-vessel regions in LWR systems. For the case of 50 vol\% iithium-spray coolant, less than $4 \%$ of the total fusion yield is deposited into the walls of the $2.6-\mathrm{m}$-radius, $0.3-\mathrm{m}-$ thick wall of the containment vessel. For a pulse rate of $0.1 \mathrm{~Hz}$, this amounts to $15.8-\mathrm{MWt}$ or $0.62 \mathrm{MWt} / \mathrm{m}^{3}$ of vessel volume. The thermal power density within a LWR pressure vessel is $\sim 5 \mathrm{MWt} / \mathrm{m}^{3}$. The energy deposited into the vessel wall during the lithium-spray blowdown has not been resolved, but, similar to the walls of most internal combustion engines, this energy must be minimized for reasons of system efficiency and vessel lifetime; this "leakage" of fusion energy that is initially deposited into the lithium spray is assumed negligible ( $\sim 1-2 \%$ of fusion yield) compared to direct nuclear heating of the blast vesse1. The 15.8-MWt low-grade energy deposited by direct nuclear heating into the vessel walls, when expressed on the basis of external surface area of the vessel, amounts to $0.15 \mathrm{MWt} / \mathrm{m}^{2}$, which is 10 times the energy naturally convected from a 32-mm-radius, 200-W incandescent light bulb. Although sufficient cooling area probably could be provided for natural- or slightly forced convection cooling of the vessel walls, forced cooling by low-temperature water has conservatively been adopted. The water coolant system is slightly pressurized in event of a shot which is not attenuated by the lithium-spray coolant to an extent predicted by the somewhat idealized neutronics model. Biological shielding located outside the blast vessel should operate with a very low power density, and probably would be cooled by natural convection or circulation occurring within the primary containment system. Since a shielding calculation, per se, has not been performed, the shielding requirement, in general, and the neutronic interaction between the biological shield and the blast vessel, in particular, remains unresolved.

(1) Vacuum is required only to an extent needed to prevent the generation of gaseous shocks within the containment vessel. Only roughing pumps should be risuired, and the capacity is estimated on the basis of roughing from $10 \pm n 1$ torr in $10 \mathrm{~s}$. The values quoted apply only to each blast vessel.

(m) Based on $0.049 \mathrm{~kg}-\mathrm{T} / \mathrm{MWt}-\mathrm{y}$ and $356 \mathrm{MWt}$ of pure fusion power, to give $17.4 \mathrm{~kg} / \mathrm{yr}$ tritium consumption, $158 \mathrm{~kg} / \mathrm{yr}$ cycled (11\% burnup), and a 1 month supply.

(n) Weight of spherical blast containment vessel of 2.6-m inner radius and $0.3-\mathrm{m}$ wall thickness. Although a detailed design of the blast vesse 1 has not been made, the $\sim 200$-tonne unit would have a $\sim 50$-tonne demountable top head through which both lithium spray and liner/leads assemblies would be irjected. The life of this vessel would be $\sim 10 \mathrm{yr}$, replacement would represent a major effort, but the vessel replacement should be considerably simpler than that for a LWR pressure vessel. Although design of the blast vessel for the plant lifetime $(\sim 30 \mathrm{yr})$ is 
TABLE III-V Cont'd.

Footnotes Cont'd.

certainly possible, the weak design and engineering basis for this system at this time as we 11 as the unique operating environment for this pressure vessel has led to the more conservative choice of a $270-\mathrm{yr}$ replacement and/or refitting period. In any case, the blast vessel is not considered a module.

(o) Shielding would be provided by concrete (steel structural support for the blast vesse1), not unlike that for the pressure vessel in a LWR system. Only a biological shielding (and a structural support) function would be performed by this system.

(p) The containment building would include a $\sim 2-m$ bic $c y^{i} i a l$ shield that encloses the blast vessel per se as well as the $\sim 1-m$-thick structural walls that enclose the liner/leads replacement room. These latter walls would have to be thick enough to provide biological shielding from post-shot radiation (from remnants of leads) as well as providing structural support for a 100-tonne crane. Refer to Fig. III-24.

(q) If oil/paper capacitors are used as transfer elements the ETS building would have to be provided with ar extensive fire-preverition system(e.g., Halon). Aside from the umbilical power feedthrough, the ETS room would not be a part of the containment room.

(r) Each of the 8 FLR units requires $390 \mathrm{MJ}$ every $10 \mathrm{~s}$; this energy would be delivered from a common homopolar $N / G$ and transfer capacitor unit, the homopolar transferring its energy to a storage inductor in $\sim 0.1 \mathrm{~s}$ and the inductor capidly ( $220 \mu \mathrm{s}$ ) transferring its energy via a transfer capacitor directly to the imploding liner. In a sense, therefore, each liner shot could be viewed as a "cold start" requiring power from the grid, at a level of $390 \mathrm{MJ} \times 8$ units $/ 10 \mathrm{~s}=312 \mathrm{MWe}$. This power, however, would be supplied continually and in proportion to the total plant outiput. Although a given unit could not accept its $\sim 39-$ MWe share of this recirculating power during the $20.1-\mathrm{s}$ period when energy is being transferred from the homopolar $M / \mathrm{l}$ set to the storage inductor, appropriate circuitry and switching can be designed that would prevent this short interruption, occurring for $\sim 0.1 \mathrm{~s}$ every $10 / 8=1.25 \mathrm{~s}$, from being "seen" by the 312-MWe recirculating power supply. A detailed circuit design of this internal power-handling system has not been made. Generally, the 312 MWe would be switched to a unit that is in a passive or stand-by state when the unit under question is being switched into the liner; otherwise, an electrical ballast or "surge-tank" would have to be used.

(s) Based on an 8-kWe power requirement to remove and reject 1 MWt of low-grade $\left(\sim 80^{\circ} \mathrm{C}\right)$ energy by water cooling.

(t) The superconducting stator windings on the homopolar $M / G$ sets will require $0.2 \%$ for every $G J$ of stored and switched energy. This loss is embedded in the 95\% transfer efficiency assumed for the ETS system. 
TABLE III-V Cont'd.

Footnotes Cont'd.

(u) Based on $100 \mathrm{kWe}$ per $25000 \mathrm{~L} / \mathrm{s}$ for Roots blowers.

(v) The $7.5 \mathrm{MJ}$ required to prepare the plasma prior to a given liner implosion is assumed to be supplied in $\sim 10 \mathrm{~s}$ with a $75 \%$ efficiency. The l-MWe/unit requirement would be supplied by a continuous 8-MWe supply, subject to an as yet unspecified ballast constraint similar to that described in footnote $(r)$.

(w) The miscellaneous plant auxiliaries have not bsen specified, but would include the power requirements of the liner/ieads debris-recovery and recycle system. This requirement is taken as $1 \%$ of the gross electrical output.

( $x$ ) Based on replacing 200-tonne blast vessel every $10 \mathrm{yr}$. 
TABLE III-VI

ESTIMATED CAPIIAL COSTS FOR THE FLR DESIGN

POINT GIVEN IN TABLE III-IV

Acrount

Number

20.

21.

22.

23.

24.

25.

26.

90.

91.1.

91.2 .

91.3.

91.

92.

93.1 .

93.2 .

93.3.

93.

94.

95.

99.
Account Title

Land and Land Rights

Structures and Site Facilities

Reactor Plant Equipment

Turbine Plant Equipment

Electric Plant Equipment

Miscellaneous Plant Equipment

Speciar Materials

Total Reactor Direct Capital Cost

Temporary Facilities

Construction Equipment

Construction Services

Construction Facilities, Equipment, and Services (15\%)

141.728

Engineering and Construction Management Services

$(15 \%) \quad 141.728$

Taxes and Insurance

Staff Training and Plant Startup

Owner's G\&A

Other Costs (5\%)

47.243

Interest During $10 \mathrm{Vr} \quad$ Construction $(8 \% / \mathrm{yr}=49.4 \%)$

821.453

Escalation During $10 \mathrm{Yr}$ Construction $(5 \% / \mathrm{yr}=33.8 \%)$

431.135

Total Reactor Capital Cost
2528.136 
SUMMARY OF CAPITAL AND OPERATING COSTS FOR THE

FLR DESIGN POINT GIVEN IN TABLE III-IV

Thermal Power (Mt)

Gross Electric Power (MWe)

Net Electric Power (MWe)

1/Recirculating Power Fraction

Plant Factor

Direct Investment Cost $(\$ / \mathrm{kWe})$

Total Investment Cost $(\$ / \mathrm{kWe})$

Capital Return 15\% (Mil1s/kWeh)

Operating $2 \%$ (Mi11s/kWeh)

Operating For Leads and Liner(Mills/kWeh)

Power Cost (Mills/kWeh)

$\begin{array}{lr}= & 3400.00 \\ = & 1360.00 \\ = & 1016.00 \\ = & 3.95 \\ = & .85 \\ = & 929.97 \\ = & 2488.32 \\ = & 50.33 \\ = & 6.71 \\ = & 10.09(\mathrm{a}) \\ = & 57.04\end{array}$

3400.00

1360.00

016.00

85

)

(a) The leads and liner replacement costs have been evaluated separately (Appendix C) using $0.10 \$ / \mathrm{kg}$ for insulator costs and $0.01 \$ / \mathrm{kg}$ for conductor recycle cost. The leads configuration that separately minimizes investment costs according to the separate algorithm is described in Appendix $C(0.2-\mathrm{m}$ radius, $0.01-\mathrm{m}$ thickness, $2.0-\mathrm{m}$ length, 0.7 conductor volume fraction). At these unit costs for insulator and conductor, the leads/liner cost would amount to $3.56 \$ /$ shot. 
Following the guidelines established for the intercomparison of alternative fusion concepts, ${ }^{45}$ the net electrical output from the FLR must be in the 1000-MWe range. To accomplish this goal, the low-yield design point requires 8 units of the type depicted in Fig. II-1. Both the values given in Table III-V and the engineering cost estimate is based on an eight-unit, $\sim 1000-M W e$ (net) system. A definite cost advantage arises by this modular approach, in that the expensive ETS system can now be shared by 8 FLR units of ح 130-MWe(net) size each; hence, for a 10-s pulse time per unit the ETS unit must discharge every $1.25 \mathrm{~s}$. The functioning of each unit follows the description given in Sec. II and Fig. II-1, and Fig. III-24 gives a schematic plan/elevation drawing of the total $\sim 1000-M W e(n e t)$ system. Two cavities drive a single Li/Na intermediate heat exchanger [17], two $\mathrm{Na} / \mathrm{H}_{2} \mathrm{O}$ heat exchangers are used, and two steam generators are envisaged. The footnotes associated with Table III- $V$ give the rationale for the engineering parameters summarized thereon.

Because of the scoping nature of this design study many of the items on Table III-V are not based upon detailed computations. Furthermore, this design point has been selected on the basis of a physics optimization that has been tempered by intuitive but realistic technological judgments. Ultimately, more detailed studies should project a design point that is optimized on the basis of a self-consistent and iterative physics, engineering, and costing model. Whether the results from such an analysis differ significantly from those given in Table III-V remains to be seen.

\section{PRESENT KNOWLEDGE IN PHYSICS AND TECHNOLOGY}

The primary intent of the FLR study is to quantify the optimum physics operating point, as embodied in Table II-I. Although a complete and selfconsistent engineering design is beyond the scope of both this study and present theoretical and experimental knowledge, crucial technological issues were identified and when possible analyzed quantitatively. Engineering computations were performed to an extent necessary to carry out a preliminary estimate of cost as well as to assess required technological development. This section concludes the FLR study by means of an assessment of present knowledge in both physics and technology. 


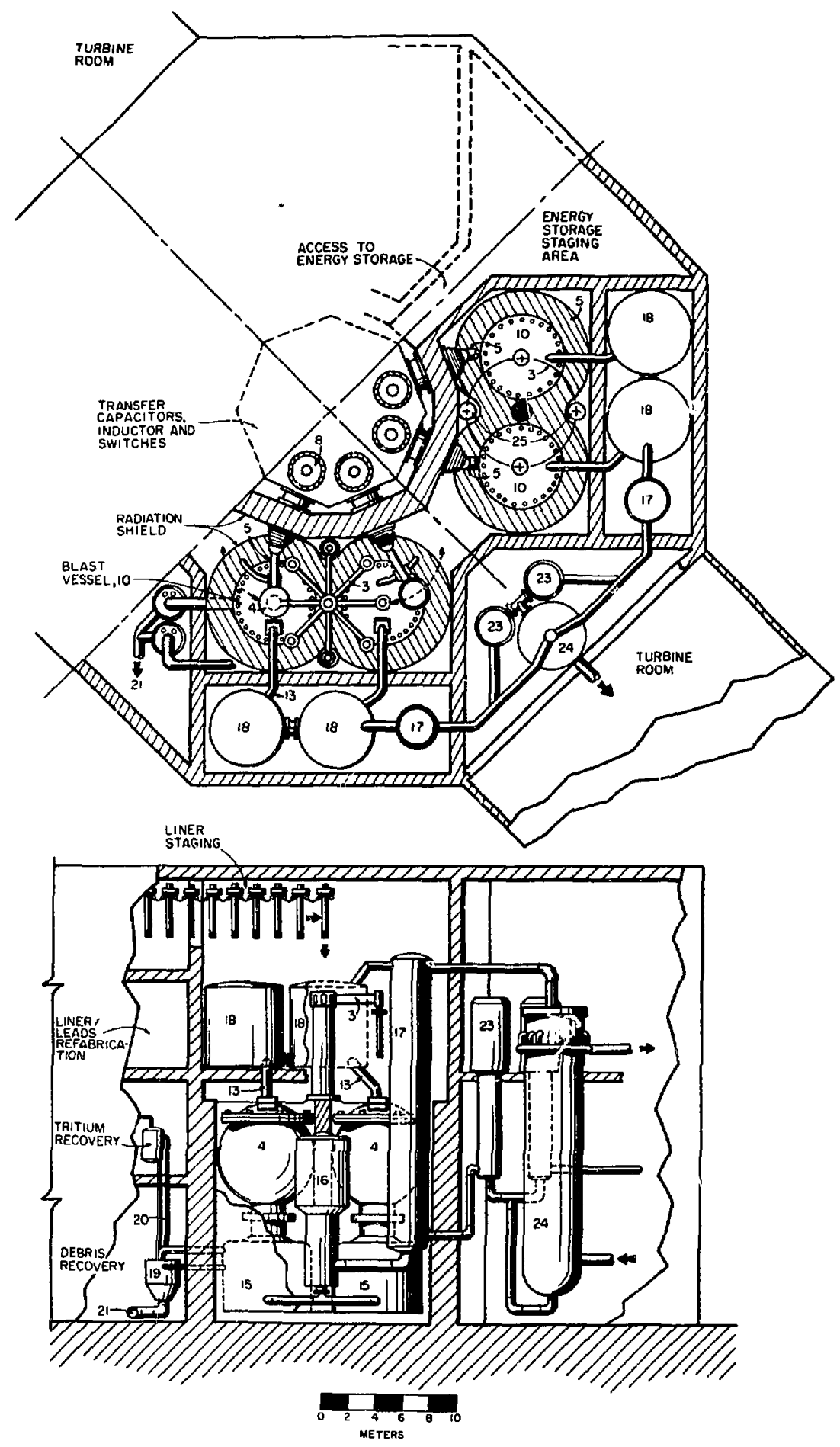

PLAN AND ELEVATION-CONCEPTUAL 8-UNIT FAST-LINER REACTOR (3400 MWi)

Fig. III-24. Plant layout for a nominally 1006-MWe(net) FLR using 8 blastconfinement cavities. Two cavities drive a separate $\mathrm{Na} / \mathrm{Li}$ heat-transport system, and two such systems drive a single $\mathrm{Na} / \mathrm{H}_{3} \mathrm{O}$ steam generator (4 cavities per steam generator, or two steam generators) component identification numbers are defined in Fig. II-I. Additionally: 23, Na pump; 24, steam generator. 


\section{A. Physics Confidence}

Since fast-liner experiments are just beginning ${ }^{5}$ and, relative to the projected FLR requirements, are at a very elementary level, predictions of reactor-grade plasma conditions are necessarily speculative. Naturally, more data are available on liner behavior than on plasma properties for the conditions envisaged for the FLR.

1. Plasma Preparation. As discussed in Sec. III.B.2, gun plasmas have been produced at densities of $2(10)^{23} \mathrm{~m}^{-3}$ with directed energies of n $0.2 \mathrm{keV}$. A fivefold increase in density and a doubling of energy would reach the necessary plasma conditions for an FLR, assuming an embedded $B_{\Theta}$ of $\sim 13 \mathrm{~T}$ can also be achieved. It seems probable that this initial condition can be reached with sufficient effort. It is not clear that the apparatus required can be economically made energy efficient and located sufficiently far from the liner to avoid damage from radiation and shock waves.

2. Transport. Plasina transport computations made to date have indicated that an FLR as envisaged here would work. This perspective could be changed radically, however, by several factors. Perhaps the most serious problem would be plasma turbulence that could sweep high-density material from the liner into the plasma and beyond the sheath of insulating magnetic field; line radiation would then quickly cool the plasma interior below acceptable levels. Lindemuth and Jarboe ${ }^{46}$ computationally observed enhanced thermal conduction to the liner $(40 \%)$ by small vortices generated near the liner wall. This computational model did not include a mechanism for losses by high-Z line radiation, however.

Another inadequacy of the current plasma model is embodied in the assumption of perfect electrical conductivity within the liner. Prediction of electrical properties of a metal under the éxtreme temperatures and pressures encountered by the liner is difficult. Best estimates are incorporated into the hydrodynamic code CHAMISA ${ }^{17}$ and should give improved estimates in the near future. More important, however, the ongoing series of LASL experiments should shed considerable light on this uncertainty.

Although confidence in general trends and scaling of plasma properties is high (Sec. III.A.4) the physical completeness of these models must be considered moderate to poor. If, for example, transport losses are more severe than anticipated by the present model, present knowledge can help alleviate the problem to the extent limited by economic and technological 
(plasma preparation, faster implosions) considerations. If, on the other hand both the conditions assumed for plasma preparation and liner drive prove to be too optimistic, while simultaneously turbulence becomes a major energy loss mechanism, the entire FLR concept may not prove feasible.

3. Liner. The liner itself is probably the best understood component central to the FLR physics. As noted above, liner implosions have been demonstrated experimentally, 6,7 and a major fast-liner project is under way at LASL. ${ }^{19}$ A variety of analytic liner models ${ }^{17,24}$ have shown goud agreement in predicting liner dynamics. Perhaps the most serious problems are the attainment of high velocities $\left(10^{4} \mathrm{~m} / \mathrm{s}\right)$ and the generation and retention of the internal insulating field, $B_{\Theta^{*}}$. These questions should be largely answered by the LASL experimental program. 19

B. Technology

If the FLR were to function within the physics predictions given in Sec. III.A and assessed in Sec. IV.A, the feasibility of the reactor system as analyzed in Sec. III.B appears technologically or economically difficult. Although the means by which the plasma is prepared, the liner/leads assembly is manipulated, and the blast is contained can be conceptualized, no credibly detailed mechanism by which to perform the crucial operations ccu?d be invented within the limits of this study. Superposing realistic economic consiraints upon the physics uncertainties renders many of these technological problems/uncertainties even more difficult. Each of these issues is discussed below in decreasing order of perceived importance.

1. Liner/Leads Fabrication. The economic implications of maintaining the cost of leads and liner below $\sim 0.04 \$ / \mathrm{kg}(6.70 \$ / \mathrm{shot})$ if the associated operating cost is to be held below $30 \%$ of the total power cost represents a crucial uncertainty for the FLR. Table IV-I summarizes 1977-78 unit costs for both fabricated items and basic materials. Clearly, the required leads/liner un it costs (70\% conductor at $0.01 \$ / \mathrm{kg}, 30 \%$ insulator at $0.10 \$ / \mathrm{kg}$ ) are far below those presently achieved by today's manufacturing industry, with possible exception of the packaging industries. On the basis of unit costs for most metals, it is concluded that the destroyed conductor must be recovered and recycled; the cost of (glass) insulator must approach that of a soft-drink container, the latter cost representing essentially an energy cost associated with the heat of fusion. Although a considerable design effort 
must be expended on the basis of more experimental data to resolve this leads/liner cost issue, this economic constraint presently appears very serious.

\section{TABLE IV-I}

TYPICAL UNIT COSTS FOR FABRICATED ITEMS AND BASIC MATERIALS

\section{Fabricated Items}

Commercial jet aircraft

Nuclear aircraft carrier

Copier

Bicycles

SS or Al piping "tee"

LWR pressure vessel

Alternatior

Diese1 generator

Passenger bus

Electric motor

Automobile

Hamburger

Soft drink bottles

Basic Mat:erials

Aluminum

Copper

Stee 1

Lead

Lithium

Lithium

Pyrex

Alumina

Mulite
Plate

Secondary ingot

Plate

Wire blank

Boiler plate

Cold roll

Ingot

Brick

Commercial (low sodium)

Chemical (99.88\%)

Large-bore tube

Powder

Medium-bore tube

Medium-bore tube
Unit Cost

$(\$ / \mathrm{kg})$

115-133

55-62

52-60

$37-60$

22-40

$11-35$

16-24

8.0-8.9

$7.7-8.2$

4.4-9.3

3.6-5.3

2.2-2.9

0.02-0.04

4.19

1.21

2.31

1.43

0.35

0.31

0.57

0.88

27.49

259.03

2.86

2.20

32.10

22.00 
2. Plasma Preparation. The means by which a $0.5-\mathrm{keV}, 1.25(10)^{24} \mathrm{~m}^{-3}$ plasma with a total energy of $7.5 \mathrm{MJ}$ is to be efficiently injected into or created within the 2.5-litre liner from a distance of $3-5 \mathrm{~m}$ is presently unproven, although a number of schemes have been addressed in Sec. III-B.2. This problem is further complicated by the need to create simultaneousiy a 213-T, azimuthal insulating field, which corresponds to a uniform current density of $120 \mathrm{MA} / \mathrm{m}^{2}$ through the injected plasma. Although laser-heated plasmas have been produced that approach these temperatures and densities, ${ }^{28}$ the required total energy, embedded insulating field, repetitive and remote production, and timing with a simultaneous liner implosion have not been demonstrated. Although an experimental basis that supports the specific needs of the FLR does not exist, the LASL experimental program will address this issue in the next few years. 5

3. Containment. The data base for the containment of explosive releases in spherical vessels has been reviewed in Appendix E (Fig. E-1). If the scaling projected by these relatively low yield experiments and predicted (for the vacuum case) by the virial theorem (Sec. III.B.6 and Appendix E) applies to the 1.5-GJ releases anticipated for the FLR, to first order, no insurmountable problem for blast confinement is anticipated. In addition to a significant extrapolation of the existing data base, the issues of shock formation in blast-mitigating media, asymetric blasts and projectile formation, propagation of damage by shocks to "safe" regions of the system (pumps, storage vessels, leads connectors, plasma preparation, etco), fatigue failure at penetrations and weldments, and focused shocks represent important issues for the containment system. The lithium-spray coolant/blast-mitigator is not an effective radiation shield for the volume fractions, vessel sizes, and chemical compositions selected (Sec. III.B.5); the structural first wall will experience an appreciable, low-energy neutron flux, although this problem is amenable to a number of design solutions. Furthermore, the actual shape and form of the first wall, insofar as the mitigation and/or time-extension of shocks via bow-wave phenomena are concerned, must be resoived by more detailed computation and experiment.

The coupling of the blast-confinement, neutronics (energy deposition, tritium breeding, shielding), and heat-transfer functions of the containmentvessel/lithium-spray system is very strong. Self-consistent calculations that simultaneously couple all these elements together have not been made, nor are 
they likely to be made in the near future. Furthermore, the liner physics and blast containment are not independent; if for reasons of turbulence or limits on plasma preparation the liner energy $W_{L}$ and associated thermonuclear release must be increased to achieve the required liner and/or engineering Q-value, the blast-containment problem will become more difficult.

4. Energy Transfer and Storage. The low-yield design point (Table II-I) requires the transfer of $\sim 450 \mathrm{MJ}$ of energy in 20-30 $\mathrm{s}$ sith an external transfer efficiency of 0.95 ; for a voltage of $\sim 200 \mathrm{kV}$, the required currents lie in the ranze 250-500 MA. The energy transfer is "one-way," and no reversible recovery is required. Table IV-II summarizes characteristics of potential energy storage systems that may be used. The selection of a slowly discharging homopolar motor generator coupled to an inductive store has been selected on the basis of a potential cost advantage, although the use of high-energy-density electrolytic capacitors may offer additional advantages, since the homopolar/inductor scheme still requires an appreciable fasttransfer capacitor bank. The switching and transfer of the required currents at the voltages needed and the desired risetimes will require significant development, considering the 10-to 20-s pulse rate, energy-focusing, and time-sequencing requirements. Because of the anticipated expense associated with the energy transfer and storage system, a single unit will have to service a number of liner cavity systems at the vi0-to 20-s repetition rate. For each cavity approximately 40 MWe will be required to charge the storage system during the $\sim 10-s$ period beiween pulses, and the associated internal power-handling requirements have yet to be addressed.

5. Leads/Liner Replacement. As noted in Sec. IV.B.1, the details of the leads/liner fabrication remain unresolved. Likewise, the means by which a 0.5-tonne leads/liner assembly is inserted into the blast containment, attached to the driving energy source, fitted with a plasma preparation unit, and subsequently removed has not been resolved. This sequence of operation must occur once every 10-20 s. For an 85\% plant factor, each 430-Mwt cavity must cycle $2.5(10)^{6}$ liner/leads assembly each year or 7300 units/d. If a total plant inventory is to remain below $\sim 100$ liner units (per cavity), the liner/leads fabrication time must be less than 20 minutes. Given that each assembly weighs $\sim 0.5$ tonne, the total liner throughput amounts to $50 \mathrm{~kg} / \mathrm{s}$ or $1.3(10)^{6}$ tonne/yr ( Li coolant flcw is $1750 \mathrm{~kg} / \mathrm{s}$ ). 
6. Primary Heat-Transfer System. The lithium-spray primary coolant system described in Sec. III.B.7, although non-conventional and untried, appears to present no intrinsic difficulties. The coupling of the transient establishment of the spray, the equilibration of the exponential energy density left in the two-phase coolant immediately after the implosion, and the influence of shocks and/or blast-mitigating processes creates some unresolved issues for this untried technology. Given that these problems can be successfully resolved within the containment vessel, no unusual difficulties are envisaged in removing the $50-60 \mathrm{~K}$ sensible heat delivered to the continuously flowing lithium cooiant. Furthermore, temperature transients at the $\mathrm{Li} / \mathrm{Na}$ heat exchanger can be virtually eliminated at the expense of an increased lithium inventory (within the sump). Given that the total lithium inventory equals $\sim 10$ times that required in the blast vessel for a single shot, the lithium inventory would amount to 0.47 tonne/MWt. It should be noted, however, that both lithium (centrifugal) pumps and Li/Na heat exchangers are not commercially available items.

TABLE IV-II

SUMMARY OF POTENTIAL. ENERGY STORAGE SYSTEMS

GENERAL FEATURES OF PULSED-POWER SUPPLIES

\begin{tabular}{c} 
Type \\
of \\
Store \\
\hline
\end{tabular}

Capacitive

Inductive

Fast Inertial (HETS)

Slow Inertial (HETS)

Very Slow Inertial

(A1 ternator, SCR-PS)

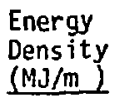

$0.01-0.10^{(a)}$

10

$100^{(b)}$

100

100

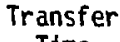

Time

ims)

$$
10^{-6}
$$

$0.1-10$.

$1-100$

$10^{3}-10^{4}$

$10^{3}-10^{4}$
Cost

$(\$ / J)$

$0.10-0.25$

$0.01-0.10$

$0.01-0.10$

$0.001-0.01$

$0.01-0.10$
Largest Installation Existing or Expected (MI)

10

$10^{2}$

$10^{3}$

$70^{4}$

$10^{4}$

(a) $0.003 \mathrm{MJ} / \mathrm{m}^{3}$ of bank energy.

(b) $1000 \mathrm{MJ} / \mathrm{m}^{3}$ has been achieved. 


\section{SUMMARY CONCLUSIONS}

This FLR study has not been a multi-man-year effort, and consequently a detailed, self-consistent design has not emerged. Furthermore, on the basis of present knowledge in both physics and technology, it is doubtful whether such a design could be generated at any level of effort at this time; the physics and engineering data base required to be applied to the many unique areas in the FLR concept simply does not exist. Nevertheless, the following conclusions seem apparent.

- Based upon the realistic physics models used to estimate the breakeven and reactor-like conditions for FLR, achievement of these conditions in the laboratory appears promising. Although the effects of thermal loss enhanced by microturbulence have not been modeled, and represent a major physics hurdle, this issue does not appear crucial to the physics success of the FLR concept at this time.

- All physics optimizations are based on a liner with the physical properties of copper, whereas preliminary cost estimates indicate that a once-through usage of copper would be economically unacceptable. Either a means must be found to recover low percentages of copper from the liquid-metal coolant or liner materials and/or configurations with properties similar to copper liners must be found.

- The feasibility and cost of the blast confinement presents no serious barrier. Although this conclusion is based upon results from approximate analytic models, sufficient design flexibility and innovation areavailable to solve unforseen blast confinement problems within realistic constraints of cost and technical feasibility.

- Both the design and cost of the FLR leads/liner structure present major problems. Although the bounds and constraints of this problem have been quantified, it is not clear that the present or readily extrapolatable technologies can deal with this problem.

- Both the physics and technology required to prepare the plasma for fastliner compression are not within reach of present or near-term knowledge. Although four possible methods were suggested, the relevant physics data base is poor, and significant experimentation is required. Since these plasma preparation requirements are specified on the basis of optimal FLR physics, these requirements can be relaxed only by degrading the reactor ergonic performance (within the limits of the models used). 
- The energy transfer and storage (ETS) requirements, including transfer efficiencies, are beyond the state of the art. Although no technological limit could be identified that would not allow the ETS goals to be achieved, the costs incurred in achieving these goals may be prohibitive.

- Although the primary heat-exchange system is non-conventional, no intrinsic cost or technological barrier to achieving Li(or LiPb) spray cooling was identified. Insofar as the shielding function of the lithium spray is concerned, the point design presented was not optimal. Questions of repetition rate, thermal load on the structural wall, projectile formation and attenuation, and coupled heat-transfer/shock processes have not been fully resolved, however.

The FLR promises a relatively small (high-power density) and economical power system. If the fast-liner approach $c$ an be made to work, breakeven and reactor-like conditions can be demonstrated at a relatively early date with modest expenditures of research dollars. Although the FLR promises reactorlike plasma performance at an early date, the development of the advanced technologies cited above may extend considerably the time to commercial power.

In concluding this study it is emphasized that a rather specific liner/ leads configurations has been adopted. The problems and/or uncertainties identified with plasma preparation, materials destruction/recycling, and plasma turbulence, therefore, may indeed be significant to magnetically driven cylindrical iiners. Improvement in the reactor embodiment may result with an approach wherein the liner components are electrically accelerated outside the blast radius and subsequently brought together without significant materials destruction. Furthermore, a plasma/liner model that is more complete and/or that has been "calibrated" with relevant experimental results could conceivably lead to enhanced yields. Generally, the rapid adiabatic compression of a wall-confined plasma appears to provide acceptable energy releases without the problems inherent in pure magnetic confinement and the basic concept deserves serious attention and refinement.

\section{ACKNOWLEDGMENTS}

We express our appreciation to the LASL personnel who have assisted this study: R. Bartholemew (structural mechanics), D. J. Dudziak (neutronics), G. Gryczkowski (numerical techniques), R. C. Malone (physics), J. Marsinall (physics, blast confinement), T. A. 0liphant (numerical techniques), A. R. Sherwood (physics), and C. E. Swannack (energy storage and transfer). 74 
APPENDIX A

DESCRIPTION OF LNRBRN CODE

A flow chart for the LNRBRN code system is shown in Fig. A-1. In addition to data input, printout, and plotting, the main program controls subroutines that perform timewise integration of the transport equations, readjust the Lagrangian mesh for pressure balance, manipulate the liner, and set the time step.

A11 time-dependent variables are stored in arrays with the subscript $K=1,2,3$ corresponding to the times $t_{j-1}, t_{j}$, and $t_{j+1}$, respectively. In the code the corresponding nomenclature is $\mathrm{JJ}-1=\mathrm{JM}=j$. All functions are known in the $\mathrm{JJ}$ loop for $K=1$ and 2 ; $D T=\Delta t=t_{j}-t_{j-1}$ is initially assumed as $t_{j+1}-t_{j}$. For the first step in the $J j$ loop cycle, subroutine PROJKT linearly projects new variables to the time $t_{j+l}, k=3$, using data from the preceding two time steps. Included in PROJKT are the coefficients of the transport equations. Subroutine LDRIVE uses known variables at time $t_{j}$ and projected or iterated variaules at $t_{j+1}$ to compute liner deceleration, velocity, position, and compression at time $t_{j+l}$, $K=3$. Subroutine STEP implicitly solves the transport equations on a rigid mesh and subsequently calls subroutine ZPB to readjust the mesh and reestablish pressure balance. The nevily obtained variables at $t_{j+1}, k=3$, are used in subroutine COEF to recompute all physical variables and transport coefficients at $t_{j+l}$. The ITA loop returns to pass through subroutine STEP two more times to improve iteratively the computed data at time $t_{j+1}$.

The time step is controlled in the ITB loop. Subroutine TIMTST computes the maximum fractional change of the plasma temperacure and field variables ( $T$ and $B)$ between $t_{j}$ and $t_{j+l}$. If this change, $f_{c}$, satisfies $4 \% \leq f<10 \%$, $\Delta t$ is not changed. If $f_{c} \leq 4 \%, \Delta t$ is increased by a factor of $2 n$ the next cycle of the $3 J$ loop. When $f_{c}>10 \%, \Delta t$ is immediately reduced by 2 , and control is returned to the start of the JJ loop. If $\Delta t$ is reduced 10 times in a JJ cycle, an error message is printed.

Subroutine ENBAL computes all energy-related functions: total plasma and field energy, liner kinetic and compressional energy, and mechanical work done by the liner on the plasma and field. Two energy checks are given for each data printout. One check compares work done by the liner on the plasma to kinetic and compressional energy changes of the liner. The other energy check 
PROGRAM LNRBRN

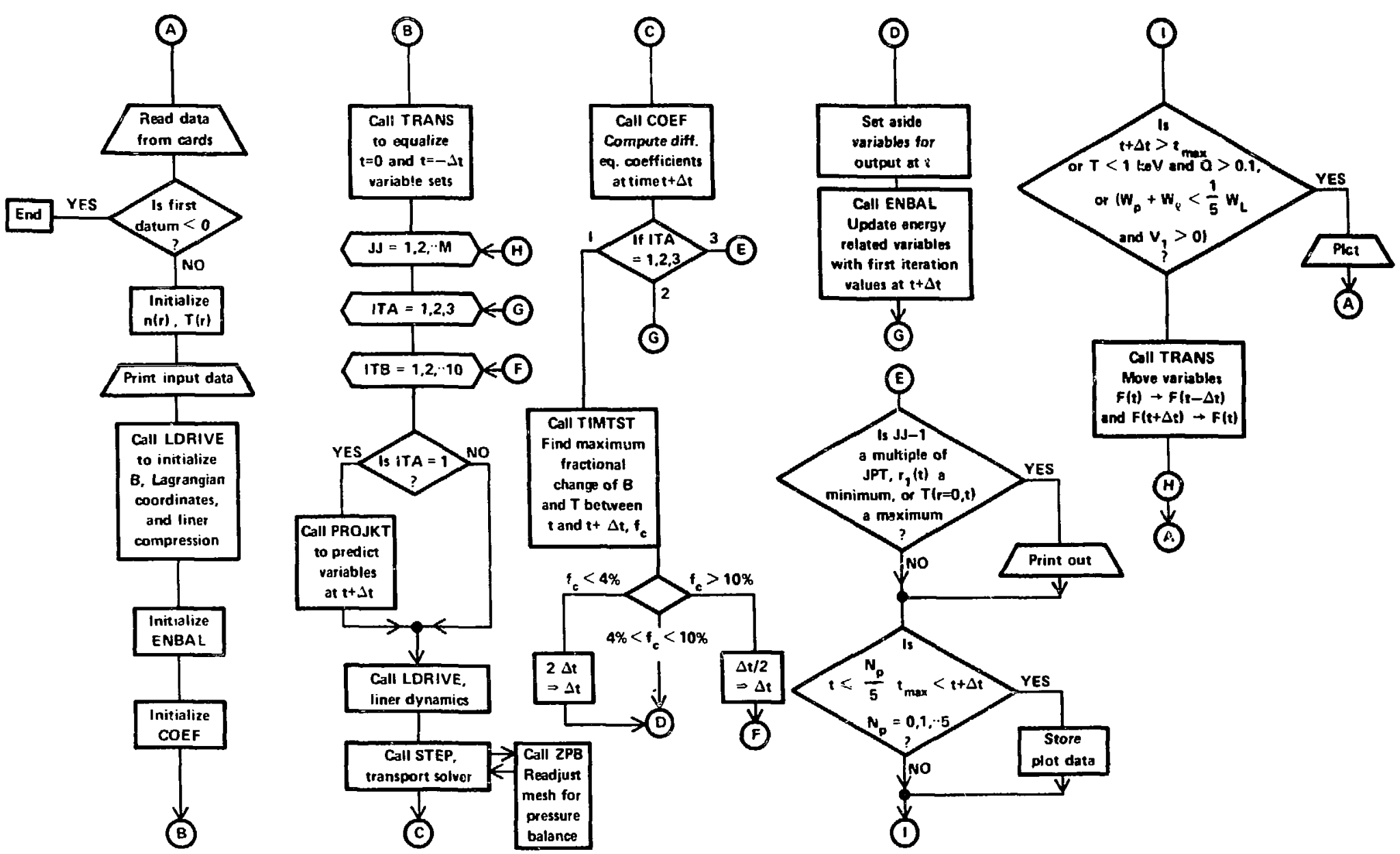

Fig. A-1. Logic flow diagram for LNRBRN: a radial, time-dependent magnetohydrostatic burn code using an analytic model for the liner compressiblity. 
compares plasina and field energy $t$, work done on the plasma-field system. A third nuriericai check is made by accounting for particles in the system as compared to the initial total minus D-T burnup.

In addition to a complete, time-dependent description of the liner and plasma parameters, the final result given by LNRBRN is presented in terms of the scientific or liner Q-value $Q=\left(W_{N}+W_{\alpha}\right) / W_{L}$, where $W_{N}$ and $W_{\alpha}$ are the fusion-neutron and alpha-particle energies, respectively. ${ }^{*}$ The liner Q-value is the object function used in all physics optimizations.

\footnotetext{
*In this study $W_{L}$ is the initial kinetic energy of an undriven 1 iner, $W$. When the liner-ETS coupling is considered, $W_{L}$ is the electrical energy transferred from the current leads to the liner assembly. Typically WkRO is $0.9 \mathrm{~W}$. This effect is not considered here, leading to somewhat optimistic Q-values.
} 
As discussed in Sec. III.B.6, a "blast radius" can be identified for the FLR inside which no apparatus is expected to survive the shock wave generated by the liner implosion. The electrical power needed to drive the liner must be transmitted from a permanent fixture outside this blast radius through destructible power leads and to the liner. In this section the cost optimization of a destructible coaxial transmission line is described. The results of this analysis of simple, coaxial leads point out a definite problem associated with the leads mass and have led to the adopition of the "forcereduced" inter leaved structure described in Sec. III.B.4 and Appendix C.

A generalized coaxial leads structure for a FLR is illustrated in Fig. B-1 and Table B-I. Three unit costs are considered: insulator $\mathrm{C}_{\mathrm{I}}(\$ / \mathrm{kg})$, conductor $c_{c}(\$ / k g)$, and energy $c_{e}(\$ / J)$. The insulator and conductor costs apply to the manufacture of new components using debris from previous liner shots as well as any make-up materials required. Energy costs apply to all energy entering the destructible transmission line that does not reach the ?iner assembly. Energy is dissipated in the leads by three mechanisms: (a) joule heating of the conductor, (b) kinetic energy imparted to the conductor by high magnetic fields, and (c) inductive energy remaining in the leads at the time of fusion energy release (unrecoverable energy). For all cases the energy dissipated in or parasitically absorbed by the destructible leads is assumed recovered in the thermal cycle; a portion $\left(n_{\mathrm{TH}}=0.4\right)$ of this energy, therefore, re-appears as electrical power. The leads energy cost, therefore, would be approximated by

$$
c_{e}(\$ / J)=\left(1-n_{T H}\right) c_{p}+n_{T H} c_{E T S},
$$

where $c_{p}(\$ / J)$ is the cost of power and $c_{E T S}(\$ / J)$ is line amortized, "pershot" cost of the energy transfer and storage system plus associated power conditioning equipment. Non-recoverable dissipation in the liner and surrounding return conductor, as weil as exiernal losses, are assumed here to be negligible. The following simplifying assumptions are made in order to carry out the coaxial leads optimization: (a) the insulator thickness $\Delta_{\mathbb{I}}$ is assumed to be the same everywhere even though the voltage would be slightly lower near the liner because of energy deposition along the leads; (b) tensile 
strength of the conductor is assumed negligible; c) the lead structure is assumed to be thin compared to the radius; and d) the conductor is thicker than an electrical skin depth, $\Delta_{c} \Delta$.

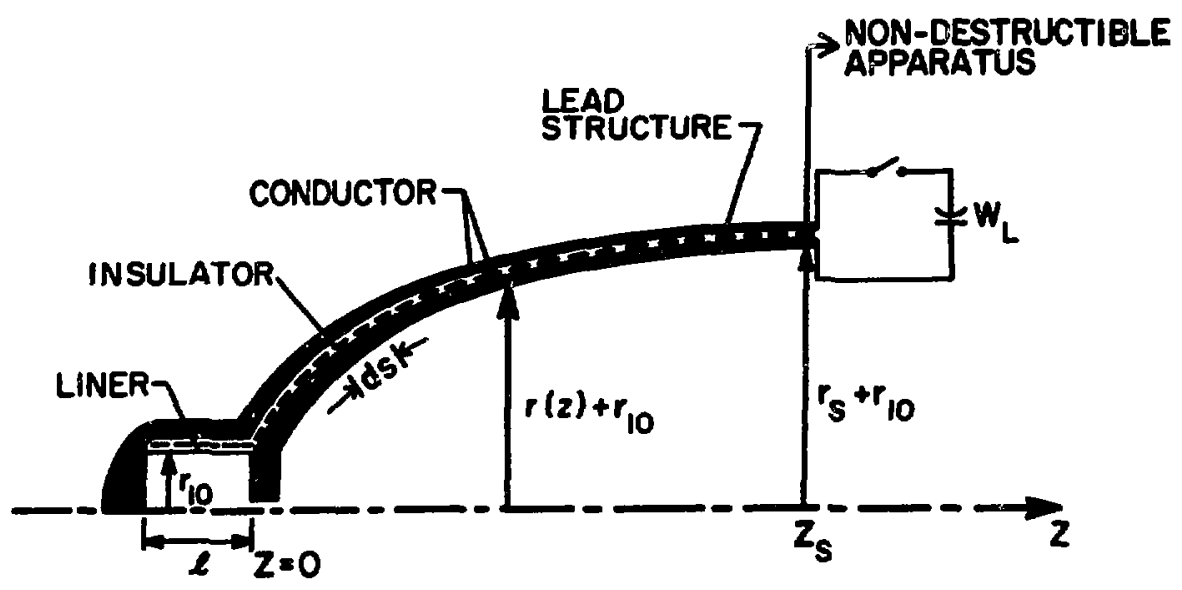

Fig. B-l. Generalized shape $r(z)$ of a coaxial leads structure used to optimize the leads structure on the basis of cost.

TABLE B-I

SUMMARY OF VARIABLES AND DEFINITIONS USED TO ARRIVE AT OPTIMUM COAXIAL LEADS CONFIGURATIONS

Symbol

$r(z)$

$\Delta_{\mathrm{B}}$

$\Delta_{c}(z)$

$\Delta_{\mathrm{C}}^{\star}$

$\Delta I$

$\Delta$

$\Delta M$

$r_{10}$

$I_{d}(t)$

$I_{\max }$

$I\left(t_{f}\right)$

$V(t)$

$t_{f}$

$W_{L}$
Definition

Lead radius at axial position $z$ measured from liner "Energy effective" conductor separation (Eq. (B-B))

Actual conductor thickness as a function of axial thickness Optimum conductor thickness (Eq. (B-17))

Insulator thickness

Electrical skin depth in conductor $=\left(2 n / \mu_{0} \omega\right)^{1 / 2}$

Movement of conductor during a puise (Eq. (B-13))

Initial liner radius

Liner drive current, $I_{\max } \sin \left(\pi t / t_{f}\right)$

Maximum Tiner current

Liner current at peak compression, $<I_{\max }$

Voltage applied to liner assembly

Time of peak compression, $\pi / \omega$

Electrical energy transferred to liner assembly 
Table B-I cont'd.

Symbol

\section{Definition}

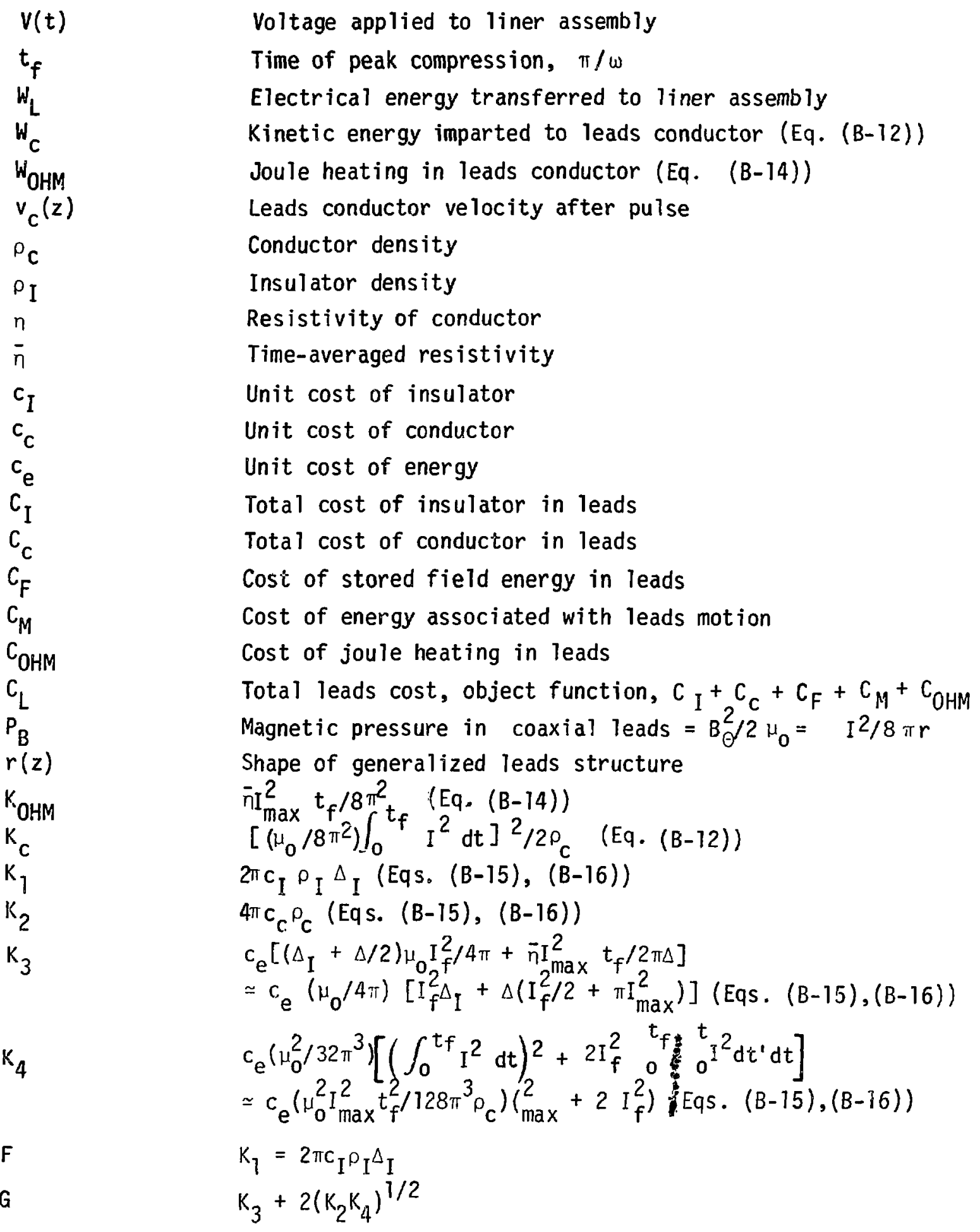


Defining ds as an element of length along an arbitrarily shaped leads geometry, the total leads cost is composed of the following components.

Insulator Cost

$$
c_{I}=2 \pi c_{I} \rho_{I} \Delta_{I} \text { Ir } d s \text {, }
$$

Conductor cost

$$
C_{c}=4 \pi c_{c} \rho_{c}{ }^{\prime} \Delta_{c} r d s \text {, }
$$

Energy cost (stored field energy)

$$
C_{F}=2 \pi c_{e} ; P_{B}\left(t_{f}\right) \Delta_{B} r d s \text {, }
$$

Kinetic energy of conductor motion

$$
C_{M}=4 \pi c_{e}{ }^{I} W_{C} r \text { ds , and }
$$

Joule heating

$$
c_{O H M}=4 \pi c_{e}{ }^{s} w_{O H M} r d s \text {, }
$$

where integration is made along the leads from the liner to a nondestructible electrical connection (Fig. B-1).

The objective of this cost optimization is to compute a trajectory $r(z)$, and conductor thickness $\Delta_{c}(z)$, which minimizes the total cost, $C_{T}=$ $C_{I}+C_{C}+C_{F}+C_{M}+C_{O H M}$, for leads connecting a liner connector ring at $\left(r_{10}, z_{2}=0\right)$ to an energy supply ring at $\left(r_{s}, z_{s}\right)$. The insulator thickness $\Delta_{I}(E q .(B-2))$ is determined by the breakdown voltage of the insulator. $P_{B}$ in $E q .(B-4)$ refers to the magnetic pressure and energy density between conductors and is given by

$$
P_{B}=B^{2} / 2 \mu_{0}=\mu_{0} I_{d}^{2} / 8 \pi^{2} r^{2} \text {. }
$$

Although $P_{B}$ varies slightly as a function of $r$ between conductors, this dependence is neglected, and $r(z)$ is taken as the centerline of the insulator. Only the field energy that remains in the leads at the end of an implosion $t$ is lost; this point is important because $I$ ( $t$ ) may be much smaller than the peak current. The "energy effective" separation of the conductors, $\Delta_{B}$, is defined as the equivalent separation of two perfect conductors at $t_{f}$. For example, it is assumed that each conductor moves a 
distance $\Delta_{M}$ during the pulse. In addition, the field energy is assumed to penetrate a conductor because of its finite resistivity. It is easily shown that this field penetration is equivalent to a conductor motion of $\Delta / 4$, where $\Delta$ is the electrical skin depth. Combining the above, the apparent separation becomes

$$
\Delta_{B}=\Delta_{I}+2 \Delta_{M}+\Delta / 2
$$

The skin depth is normally computed for a constant, uniform resistivity and a sinusoidal current of angular frequency $w$,

$$
\Delta=\sqrt{2 n / \mu_{0} \omega}
$$

For the case of the FLR only a single pulse of duration $t_{f}$ is of concern, and the resistivity varies in both space and time; joule heating is sufficient to melt a portion of the conductor. For simplicity an averaged value of $\eta$ is taken and $\omega$ is replaced by $\pi / t_{f}$ to give

$$
\Delta=\sqrt{2 n t_{f} / \pi \mu_{0}} \text {, }
$$

Conductor motion, including $\Delta_{M}$, will be discussed subsequently. In the optimized system $\Delta_{M}$ should be the same anyplace along the leads conductor; hence, $\Delta_{B}$ is fixed.

Proceeding to Eq. (B-5), conductor motion is computed using the impulse momentum approximation 36,37

$$
v_{c}=\left(1 / \rho_{c} \Delta_{c}\right) \int_{0}^{t_{f}} P_{B} d t .
$$

The corresponding kinetic energy per unit surface area of conductor is given by

$$
\begin{aligned}
W_{c} & =\rho_{c} \Delta_{c} v_{c}^{2} / 2 \\
& =\left[\left(\mu_{0} / 8 \pi^{2} r^{2}\right) \int_{0}^{t_{f}} I_{d}^{2} d t\right]^{2} / 2 \rho_{c} \Delta_{c} \\
& \equiv K_{c} / \Delta_{c} r^{4} .
\end{aligned}
$$

Likewise conductor displacement can be computed from

$$
\Delta_{M}=\int_{0}^{t_{f}} v_{c} d t \text {. }
$$


Joule heating is approximated by a simple skin-depth model

$$
\begin{aligned}
W_{O H M} & \simeq\left[\int_{0}^{\dot{t}_{f}} n I^{2} d t\right] /\left(4 \pi^{2} \Delta r^{2}\right) \\
& \simeq \bar{n} I_{\max }^{2} t_{f} /\left(8 \pi^{2} \Delta r^{2}\right) \\
& \equiv K_{O H M} / r^{2}
\end{aligned}
$$

Equations (B-2)-(B-6) are combined and rewritten to form a single object function for total leads cost

$$
\begin{aligned}
c_{L} & =2 \pi \int\left[r\left(c_{I} \rho_{I} \Delta_{I}+2 c_{c} \rho_{c} \Delta_{c}\right)\right. \\
& +\left(\mu_{0} I^{2} \Delta_{B} / 8 \pi^{2}+2 K_{0 H M}\right) / r \\
& \left.+2 k_{c} / r^{3} \Delta_{c}\right] d s .
\end{aligned}
$$

Consideration of Eqs. $(B-8),(B-11)$, and (B-13) indicates that the only variables to be optimized in Eq. $(B-15)$ are $r$ and $\Delta_{C}$; in this case the object function can be reconstituted as follows

$$
c_{L}=\int\left[r\left(k_{1}+K_{2} \Delta_{c}\right)+k_{3} / r+k_{4} / \Delta_{c} r^{3}\right] d s,
$$

where $K_{1}, K_{2}, K_{3}$, and $K_{4}$ are appropriately defined parameters (Table $B-I)$. For a given leads geometry $r(z)$, the total cost $C_{L}$ shows a minimum as the conductor thickness increases: for small thicknesses, ${ }^{\Delta} c$, considerable kinetic energy is imparted to the leads, whereas for large thicknesses the conductor materials cost dominates. More specifically, the $K_{1}$ 'cerm in Eq. (B-16) represents the insulator and/or refabrication cost, the $K_{2}$ term is associated with the conductor and/or refabrication cost, the $K_{3}$ term corresponds to joule heating (neglecting inductive energy stored in the loads at time $t_{f}$ ), and the $k_{4}$ term represents kinetic energy imparted to the leads (again neglecting stored inductive energy at time $t_{f}$ ). Generally, $I_{d}(t) \simeq I_{\max } \sin \pi t / t_{f}$, so that at time $t_{f}$ little current or inductive energy resides in the leads. This leads cost is now optimized with respect to the conductor thickness $\Delta_{c}$. 
On differentiating the integrand of $\mathrm{Eq} .(\mathrm{B}-16)$ with respect to $\Delta_{C}$ and solving for the optimum conductor thickness $\Delta_{C}^{*}$

$$
\Delta_{c}^{\star}=\sqrt{k_{4} / K_{2} / r^{2}}
$$

It is easily shown that Eq. $(B-17)$ implies that at $\Delta_{C}=\Delta_{C}^{*}$ the conductor and kinetic energy costs are equal. The object iunction $C_{L}$ is reduced to the following expression upon substitution of $\Delta_{c}^{*}$ for $\Delta_{c}$

$$
\begin{aligned}
C_{L} & =\left[r K_{1}+\left(K_{3}+2 \sqrt{k_{2} K_{4}}\right) / r\right] d s \\
& =\int(F r+G / r) d s
\end{aligned}
$$

A final simplification is to define an optimum radius $r^{\star} \equiv \sqrt{G / F}$, and the object function becomes

$$
C_{L}=F \int\left(r+r^{\star 2} / r\right) d s
$$

The designation as "optimum radius" was given to $r^{*}=\sqrt{G / F}$ because the optimal radius for a long coaxial cable equals $r^{*}$, in that this radius minimizes the integrand of Eq. (B-19). For a short coaxial cable, such as connects $\left(r_{0}, 0\right)$ and $\left(r_{s}, z_{s}\right)$ in Fig. B-1, an optimum trajectory for Eq. (B-19) must be found using the variational principle.

To minimize Eq. (B-19) with respect to $r$, the variable of integration is changed to $z$, and the first variation is taken, where $r^{\prime}$ designates the derivative with respect to $z$.

$$
\begin{aligned}
& \delta \int_{0}^{z} s\left(r+r^{\star 2} / r\right)\left(1+r^{\prime 2}\right)^{1 / 2} d z \\
= & \int_{0}^{z} s\left[\left(1-r^{\star 2} / r^{2}\right) \delta r+\left(r+r^{\star 2} / r\right) r^{\prime} \delta r^{\prime} /\left(1+r^{\prime 2}\right)\right]\left(1+r^{\prime 2}\right)^{1 / 2} d z \\
= & \int_{0}^{z} s\left[\left(1-r^{\star 2} / r^{2}\right) / 1+r^{\prime 2}\right) \\
& \left.-\left(1+r^{\star 2} / r\right) r^{\prime \prime} /\left(1+r^{\prime 2}\right)^{2}\right]_{\delta} r\left(1+r^{\prime 2}\right)^{1 / 2} d z \\
+ & {\left[\left(r+r^{* 2} / r\right) r^{\prime} \delta r /\left(1+r^{\prime 2}\right)^{1 / 2}\right]_{0}^{z} s=0 }
\end{aligned}
$$


The resulting Euler equation is

$$
\left(r+r^{\star 2} / r\right) r^{\prime \prime}-\left(1-r^{\star 2} / r^{2}\right)\left(1+r^{\prime 2}\right)=0 \text {. }
$$

In solving this equation two boundary conditions are applied when the leads are specified to connect both to the liner at $\left(r_{10}, 0\right)$ and to the boundary that delinates the blast zone $\left(r_{s}, z_{s}\right)$. Equation $(B-21)$ was solved numerically, and the results are plotted in $(r, z)$ space normalized to the initial liner radius $r_{10}$ (Fig. B-2). Each plot has been constructer for a range of normalized total costs $C_{L}$, expressed as $\zeta=C / r_{10}^{2}{ }_{10}{ }^{\rho} I^{\Delta}$.

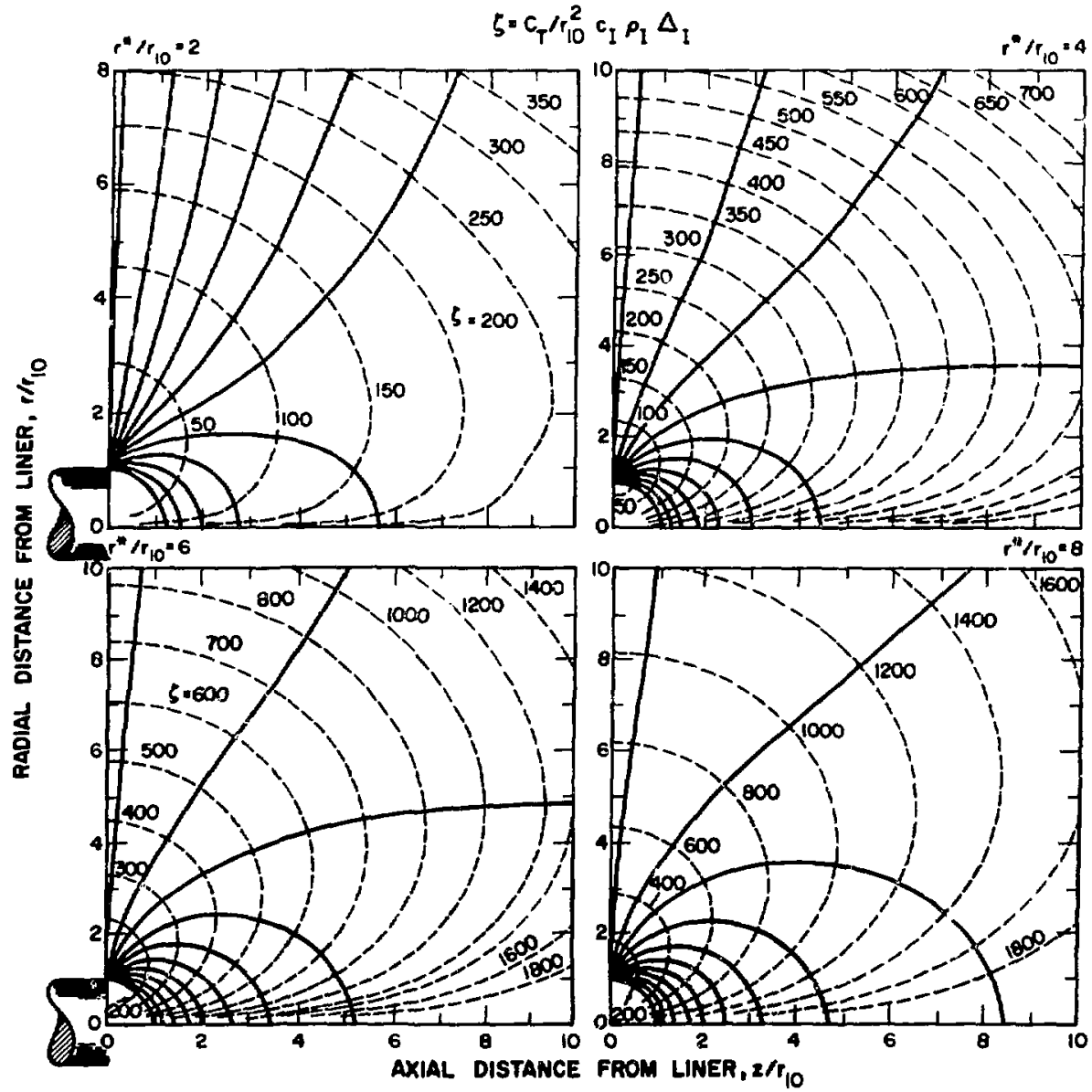
Fig. B-2. Cost-optimized coaxial lead shapes for four values of $r * / r 10$
(Eq.2 (B-19)) $c_{T} / r_{10} c_{I} \Delta_{I} \rho_{I}$, are shown. 
The remaining cost and physical parameters are embodied in the ratio $r / r$. $=$ $\sqrt{G / F}=\left[K_{3} / K_{1}+2\left(K_{2} K_{4}\right)^{1 / 2} / K_{1}\right]^{1 / 2}$ (re: Table $\left.B-I\right)$, which is taken as 2, 4, 6 , and 8 in Fig. B-2. The dashed curves in Fig. B-2 are drawn orthogonal to the leads shape curves $r(z)$; these curves represent lines of constant $\zeta=C_{L}$ ' $r_{10}^{2} c_{I} \rho_{I} \Delta_{I}$; the iso- $\zeta$ curves begin at the $\left(r / r_{10}=1, z=0\right)$ point and expand outward from this point in constant increments of $\Delta \zeta=50$. Hence, for the conditions adopted in Sec. III.B.4 and Appendix $C\left(r_{10}=0.2 \mathrm{~m}, c_{I}=0.10 \$ / \mathrm{kg}\right.$, $\left.\rho_{I}=2.5(10)^{3} \mathrm{~kg} / \mathrm{m}^{3}, \quad \Delta_{\mathrm{I}}=V / E_{\mathrm{D}}=2(10)^{5}(\mathrm{~V}) / 4(10)^{7}(\mathrm{~V} / \mathrm{m})=5(10)^{3} \mathrm{~m}\right)$ each iso- $\zeta$ curve corresponds to an incremental increase in $\tau_{L}$ equal to $2.50 \$ /$ shot.

Table B-II sumarizes typical cost and physical parainters to give physical significance to the four assumed values of $r$ ir 10 used to generate Fig. $(B-2)$. For the assumed values given on Table B-II, $r^{*} / r_{10}=2.9$.

The following points can be made with respect to the trade-offs embodied in this coaxial leads optimization. First, conductor refabrication costs and the cost of imparting parasitic kinetic energy to the leads are equally costly (Eq. (B-17) and associated discussion). By varying the conductor thickness $\Delta_{C}$, rather than selecting the optimum value $\Delta_{C}^{*}$, the total $\operatorname{cost} C_{L}$ will vary to second order with $\Delta_{c}\left(i . e .\right.$, as $\left.\Delta_{c}^{2}\right)$. Second, for the case where $r^{\star} / r_{10}=1$ (typically, $r^{\star} / r_{10} \simeq 2-3$, Table B-II), the leads cost is partitioned as follows: insulator (50\%), conductor (18\%), joule heating $(14 \%)$, and kinetic energy (18\%). Significantly, half of the cost of the coaxial leads per unit length is associated with insulator cost for $r_{10}=r^{*}(0.57 \mathrm{~m}$ for the typical values assumed in Table B-1i). For greater initial liner radii, the insulator costs will dominate. As $r_{10}$ is decreased below $r^{*}$, the conductor, joule heating, and kinetic energy costs will dominate the leads cost. Third, for straight leads sections with $r_{10}=r^{*}, \quad \partial C_{L} / \partial r=0$ and $\partial^{2} C_{L} / \partial r^{2}>0$; hence, $C_{L}$ increases as $r$ departs significantly from $r^{*}$. Last, as seen from Fig. (B-2), the most economical, close access to the liner is provided by radial feedplates. For $r_{10}=0.2 \mathrm{~m}$, these figures go to a maximum radial and axial extent of $2 \mathrm{~m}$. Interpolating Figs. (B-2) for $r^{*} / r_{10}=2.9$ (Table $\left.B-I I\right)$, using $2.50 \$ /$ shot for every increment of 50 in the parameter $\zeta, 20 \$ /$ shot would be required to operate a 2-m-radius feedplate or $23 \$ /$ shot would be expended for 2- $m-1$ ong, shaped coaxial lead with a $0.5-\mathrm{m}$ radius at the outer or power connection. 
For larger blast radii the coaxial lead would become more economical than the radial feedplate, whereas the radial feedplate would be the economic choice for smaller blast radii.

Although it has been shown that an optimization of a generalized coaxial feedplate is possible, these optimal leads costs per shot are excessive when realistic blast radii are imposed. Total leads cost on the order of 20-25 $\$ /$ shot would be incurred for these cost-optimized cases; these best cases for the coaxial lead structure are about twice as expensive as the "force-reduced" interleaved structure discussed in Sec. III.B.4. In essence, the "forcereduced" design minimizes the liner mass needed to maintain inertially the leads structure intact during the pulse as well as keeping the internal stored energy to a minimum. The interleaved leads may also be confined to a small tube as compared to the bulky, shaped coaxial leads in Fig. B-2. The cost and handiing advantages associated with the interleaved leads approach, however, must be weighed against the inherently more complex structure, which was not factored into the analysis in terms of a potentially higher fabrication cost.

\begin{tabular}{|c|c|c|}
\hline $\begin{array}{l}\text { TABLE 8-11 } \\
\text { SLMWARY OF COST AND PHYSICAL PARMETERS } \\
\text { THE DEPENDENCE OF THE ORIIMUM LEAOS CONFI } \\
\text { PARAMETER } r^{\star} / r \text { USED IN FIG. }\end{array}$ & $\begin{array}{l}\text { OT EV ELLUATE } \\
\text { ATIONS ON THE }\end{array}$ & \\
\hline Definition & Value & \\
\hline Time to final compression, $t_{f}(s)$ & $2(10)^{-5}$ & \\
\hline Peak voltage, $v_{\max }(v)$ & $2(10)^{5}$ & \\
\hline Peak current, $I_{\max }(A)$ & $2.5(10)^{8}$ & \\
\hline Time-average resistivity, in (ohm m) & $1.0(10)^{-6}$ & \\
\hline Insulator density, $o_{I}\left(\mathrm{~kg} / \mathrm{m}^{3}\right)$ & $2.5(10)^{3}$ & \\
\hline Conductor density, $\rho_{c}\left(\mathrm{~kg} / \mathrm{m}^{3}\right)$ & $1.0(10)^{4}$ & \\
\hline Dielectric breakdown strength of insulator $E_{0}(\mathrm{~V} / \mathrm{m})$ & $4(10)^{7}$ & \\
\hline Insulator thickness, $\Delta_{\mathrm{I}}(\mathrm{m})=v_{\max } / E_{D}$ & $5(10)^{-3}$ & \\
\hline Unit cost of insulator, $c_{I}(\$ / \mathrm{kg})$ & 0.10 & \\
\hline Unit cost of (recycled) conductor, $c_{c}(\$ / \mathrm{kg})$ & 0.01 & \\
\hline Energy cost $c_{e}(\$ / J)$ & $1.11(10)$ & (40 mills/kweh) \\
\hline Initial liner radius, $r_{10}(\mathrm{~m})$ & 0.2 & \\
\hline \multicolumn{3}{|l|}{$\begin{array}{l}\text { Evaluated constraints: } \\
\qquad F=K_{1}=2 c_{I} a_{I} \Delta_{I}=7.85 \mathrm{~s} / \mathrm{m}^{2}\end{array}$} \\
\hline \multicolumn{3}{|l|}{$K_{2}=4 \pi c_{c} \rho_{c}=1257 \mathrm{~s} / \mathrm{m}^{3}$} \\
\hline \multicolumn{3}{|l|}{$=\left(2 \tilde{n} \mathrm{t}_{\mathrm{f}} / \pi \mu_{0}\right)^{1 / 2}=3.2(10)^{-3} \mathrm{~m}$} \\
\hline \multicolumn{3}{|l|}{$K_{3}=c_{e}^{\mu}{ }_{o} I_{\max }^{2} \Delta_{/ 4}=0.70 \$$} \\
\hline \multicolumn{3}{|l|}{$K_{4}=c_{e} \mu_{0}^{2} I_{\max }^{2} t_{f}^{2} /\left(128 \pi^{3} o_{c}\right)=6.9(10)^{-4} \mathrm{sm}^{3}$} \\
\hline \multicolumn{3}{|l|}{$r^{\star}=0.57 \mathrm{~m}$} \\
\hline
\end{tabular}




\section{APPENDIX C \\ OPTIMIZATION OF INTERLEAVED LEADS STRUCTURE}

Although the FLR study has not progressed to a point where a comprehensive cost optimization can be made, the destruction of liner and leads structure is recognized as a potential and serious economic problem. The relatively complex lead and liner assembly depicted in Fig. III-10 is assumed to be $\mathrm{Pb}, \mathrm{LiPb}$, or $\mathrm{Li}$, and once destroyed melts into and becomes a part of the $L i$ or $L i P b$ coolant; the conductor cost is envisaged as one of refabrication by a co-extrusion process rather than a materials cost. The insulator, on the other hand, probably will not be amenable to direct recycle, it will appear as a slag on the coolant surface in the sump (14, Fig. II-1) after each shot, and it will probably represent a major fabrication cost. The simple economic optimization of the interwoven leads structure, therefore, focuses parametrically onto the fabrication cost allowed for the insulator. This optimization uses as an object function the total cost of electricity as a function of major leads parameters and a composite total plant cost; the liner cost is assumed to be a small fraction of the total cost of the leads/liner assembly.

In addition to the cost of leads material destroyed, the ohmic heating in the lead structure represents another cost penalty. Although this ohmic-heating energy requirement per se is reflested in a higher recirculating power requirement and, therefore, is not charged directly to the leads cost, the additional capital investment associated with the added energy transfer and storage (ETS) required to supply the ohmic loss does appear as a direct leads cost. If the voltage $V$ applied to the leads appears primarily to supply resistive-like liner elements (i.e., time-varying liner inductance), then $V$ $=I_{d} \ell \mu_{0} / 2 \pi t_{f}$, where $\ell$ is the length of the liner, $I_{d}$ is the liner drive current, and $t_{f}$ is the liner "run-in" time. Hence, the energy delivered to the liner $W_{L}(J)$ and the liner Q-value are given by

$$
\begin{aligned}
& W_{L} \simeq I^{2} \mu_{0} l / 2 \\
& Q \simeq \xi\left(W_{L} / l\right)^{1 / 2}
\end{aligned}
$$

where the scaling parameter $\xi$ is obtained from separate analytic and numerical computations of the liner physics described in Sec. III.A and Ref. 17. 
If $A=\pi\left(r_{0}^{2}-r_{j}^{2}\right)$ is the total cross-sectional area of the leads (re: Fig. III-10), $\lambda$ is the conductor (volume) filling fraction, $n$ is the conductor resistivity, and $\Delta=2\left(n \tau / \mu_{0}\right)^{1 / 2}$ is the conductor width (two skin depths, Fig. III-10), the energy deposited into the leads as ohmic heating equals

$$
W_{O H M}(J) \simeq 2 \pi W_{L} \Delta^{2} R / \ell A_{C},
$$

where $R$ is the length of destroyed leads (equal to a fraction, 0.3-0.5, of the blast radius) and $A_{C}=\lambda A$ is the conductor area.

The virial theorem ${ }^{\mathcal{Z}}, 38$ is used to estimate the blast radius $R$ required to contain any explosive energy release equal to $W_{L}+W_{\alpha}$, where $W_{\alpha}$ is the alpha-particle yield. For a spherical vessel of wall thickness $\Delta R$ and allowable (fatigue) stress $\sigma$,

$$
R_{B}^{2} \Delta R \geq\left(W_{L}+W_{\alpha}\right) / 4 \pi \sigma
$$

Given that $C_{E T S}(\$ / J)$ is the amortized, "per-shot" cost of the ETS system, $c_{c}(\$ / \mathrm{kg})$ is the conductor or metal refurbishing cost, and $c_{I}(\$ / \mathrm{kg})$ is a similar cost for insulator, the cost per shot of the leads structure becomes

$$
C_{L}(\$)=2 \pi\left(W_{L} / \ell\right)\left(\Delta{ }^{2} R / A_{c}\right) c_{E T S}+\left[\rho_{c} c_{c}+\rho_{I} c_{I}(1-\lambda) / \lambda\right] A_{c} R,
$$

where, if $E_{D}(V / m)$ is the dielectric strength of the insulator, $\lambda=1 /\left(1+V / \Delta E_{D}\right)$. On the basis of Eq. $(C-5)$ an optimum leads area, $A$ or $A_{C}=\lambda A$, is evident; small $A_{C}$ reflects a high ETS cost and large $A_{C}$ reflects a high materials cost. If $W_{E}(J)$ represents the net electrical energy generated by each implosion, then the electrical cost associated with the leads is simply $C_{L} / W_{E}$. Given that the total plant capital investment is $P_{I}(\$ / W e), P_{I}$ is the annual return on investment, and that $\sim \pi(10)^{7}$ seconds equal a year, the total energy cost can be approximated by

$$
c_{E}(\$ / J)=\left[P_{I} p_{I} / \pi(10)^{7}+c_{L} / W_{E T}\right] /(1-\varepsilon)
$$

where $W_{E T}=W_{E} /(1-\varepsilon)$ is the total electrical energy generated per implosion, and the recirculating power iraction $\varepsilon$, according to Fig. III-7, and 
Eq. (III-18) is given by

$$
1 / \varepsilon=Q_{E}=\eta_{T}^{E X} \eta_{T H}\left[\eta_{T}^{I N T} Q(0.2+0.8 M)+1\right] /\left(1+f_{p O}+f_{A U X}\right) .
$$

It is easily shown that the internal transfer efficiency is $\eta_{T}^{I N T}$ given by

$$
W_{O H M} / W_{L}=1 / n_{T}^{I N T}-1=2 \pi \Delta^{2} R / \ell A_{C} \text {. }
$$

Substitution of Eqs. (C-8), (C-7), and (C-5) into Eq. (C-6) gives the following expression for the cost of energy, $c_{E}(\$ / J)$, which serves here as an object function to be minimized with respect to leads configuration.

$$
c_{E}(\$ / J)=n{ }_{T}^{E X}\left[\alpha_{1}+\alpha_{2} A_{C}+\alpha_{3} A_{C}^{2}\right] /\left[\alpha_{4} A_{5}-\alpha_{5}\right],
$$

where

$$
\begin{aligned}
& \alpha_{1}=\left[\eta_{T H} P_{I} p_{I} / \pi(10)^{7}+c_{E T S}\right] 2 \pi \Delta^{2} R / 2 \\
& \alpha_{2}=\left[\eta_{T H} P_{I} p_{I} / \pi(10)^{7}\right][1+(.8 M+0.2) Q] \\
& \alpha_{3}=R\left[\rho_{C} c_{C}+\rho_{I} c_{I}(1-\lambda) / \lambda\right] \xi^{2} / Q^{2} \ell \\
& \alpha_{4}=n \frac{E X}{T} \eta_{T H}[1+(.8 M \cdots .2) Q]-1 \\
& \alpha_{5}=2 \pi \Delta^{2} R\left[1-n{ }_{T}^{E X} n_{T H}\right] / \ell
\end{aligned}
$$

Differentiation of Eq. (C-9) with respect to $A_{C}$ gives the following expression for the cost optimized leads area $A_{c}=\lambda A$

$$
A_{C}^{*}=\left(\alpha_{5} / \alpha_{4}\right)\left[i+\sqrt{1+\alpha_{4}\left(\alpha_{4} \alpha_{1}+\alpha_{2} \alpha_{5}\right) / \alpha_{3} \alpha_{5}^{2}}\right]
$$

The optimized total energy costs (Eqs. (C-9) and $(C-10)$ ), the fraction of these costs associated with the leads, and the associaced engineering Q-value $Q_{E}$ (Eq. (C-7)) are evaluated parametrically as a function of insulator cost $c_{I}(\$ / \mathrm{kg})$ on Fig. $\mathrm{C}-1$ for the fixed parameters given on Table $\mathrm{C}-\mathrm{I}$.

Generally, the cost-optimized leads areas given by Eq. (C-10) will resuit in melting of the lead conductor some time into the energy transfer, and the 
appropriate liquid resistivity was used. If on the other hand $A_{C}$ was specified to assure that melting occurred only after the energy transfer, then $A_{C}$ is given by

$$
A_{C}=\left[2 \pi W_{L} \Delta^{2} R \Delta H_{M} \rho_{C}\right]^{1 / 2} \text {, }
$$

where $H_{M}(\mathrm{~J} / \mathrm{kg})$ is the energy required to melt the conductor starting from $300 \mathrm{~K}$. The dependence of $\mathrm{C}_{E}(\$ / \mathrm{J})$ and the fraction of $\mathrm{C}_{E}(\$ / \mathrm{J})$ attributable to leads cost is also shown on Fig. $C-l$ as a function of insulator cost. For the range of liner energies $W_{L}$ and optimization procedures used as a bas is for the data on Fig. C-I, it appears that for either melting or non-melting leads options the insulator cost must be kept below $c_{I} \simeq 0.10 \$ / \mathrm{kg}$ if the FLR plant efficiency and economic viability are not to be compromised. Generally, the cost of glass-like insulator fabrication in simple but mass-profuced geometries is expected to be near the energy cost associated with meiting, ${ }^{47}$ which for methane amounts to $0.02 \$ / \mathrm{kg}$ at present costs.

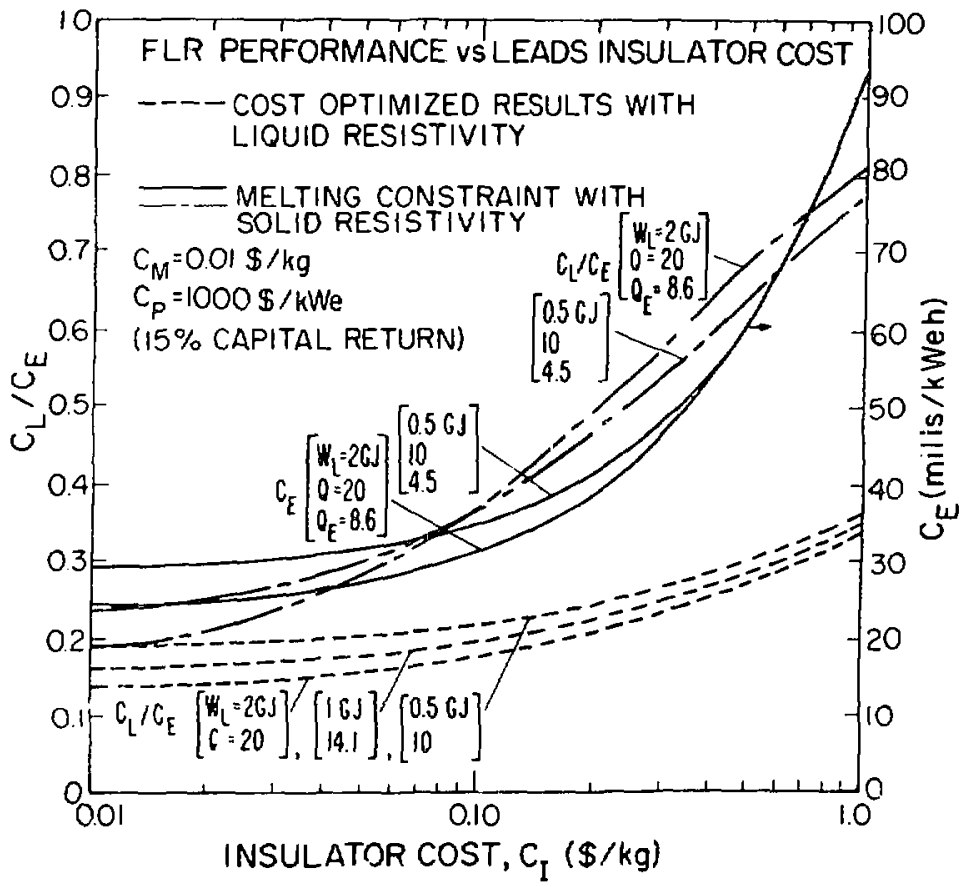

Fig. C-1. Dependence of leads cost relative to power cost on the unit cost of insulator for a fixed cost of conductor recycle $(0.01 \$ / \mathrm{kg})$. Cost-optimized and non-melting constraint are imposed. The actual power costs are also shown for the non-melting constraint imposed. 
Conductor (lead) resistivity, n(ohm-m)

Liner "run-in" time, $t_{f}(s)$

Cycle time, ${ }^{\tau}{ }_{c}(s)$

Maximum siress in blast vesse $1, \sigma(\mathrm{Pa})$

$B$ last vessel thickness, $\Delta R(m)$

$B$ last radius fraction, $R / R_{B}$

Liner length, $\ell(m)$

Conductor density, $\rho_{c}\left(\mathrm{~kg} / \mathrm{m}^{3}\right)$

Insulator density, $\rho_{I}\left(\mathrm{~kg} / \mathrm{m}^{3}\right)$

Melting energy for corductor, $\Delta H_{M C}(\mathrm{~J} / \mathrm{kg})$

Melting energy for insulator, $\Delta H_{M I}(\mathrm{~J} / \mathrm{kg})$

Insulator dielectric strength, $E_{D}(\mathrm{~V} / \mathrm{m})$

Voltage applied to leads, $V(V)$

Conductor volume fraction, $\lambda$

Cost of metal, $c_{c}(\$ / \mathrm{kg})$

Installed cost of energy storage, $\mathrm{C}_{\mathrm{ETS}}(\$ / \mathrm{J})$

Plant costs, $\mathrm{P}_{\mathrm{I}}\left(\$ / \mathrm{W}_{\mathrm{e}}\right)$

Return on capital, $\mathrm{p}_{\mathrm{I}}(1 / \mathrm{y})$

Thermal conversion efficiency, ${ }^{n} \mathrm{TH}$

External ETS transfer efficiency, $n_{T}$ EX

$B 1$ anket neutron energy multiplication, $M$

Scaling parameter for liner yield, $\xi(M / J)$

Auxiliary power fraction, $f_{A U X}=W_{A U X} / W_{E T S}$

Plasma preparation fraction, $f_{P O}=W_{P O} / W_{E T S}$
$2(10)^{-7}$ (solid), $1(10)^{-6}$ (liquid)

$45 .(10)^{-6}$

10.

$68(10)^{6}$

0.3

0.5

0.2

$10.5(10)^{3}$

$2.5(10)^{3}$

$6.34(10)^{4}$

$1.41(10)^{6}$

$4.0(10)^{7}$

$2.5 \times 10^{5}$

0.7

0.01

0.01

1.0

0.15

0.4

0.95

1.25

$2.0(10)^{-4}$

0.06

0.04 


\section{APPENDIX D \\ DESCRIPTION MCNP MCNTE CARLO CALCULATION}

The Monte Carlo code is the continuous energy code MCNP. ${ }^{31}$ Any number (limited only by the storage capabiiities of the computer) of geometric cells bounded by first- and second-degree surfaces, as well as some fourth-degree surfaces, can be treated by the code. The subdivision of the physical system into cells is not necessarily governed by the different material regions occurring, but may take into consideration the problems of sampling as well as the restrictions necessary to specify a unique geometry.

Included in the code are standard variance-reducing techniques, which are optional. These include particle-splitting and Russian-roulette and pathlength stretching techniques. Provision is made to force collisions in designated ceils, thereby obtaining flux estimates at point detectors; provisions are also made for calculating reactions in small regions for use as tracklength estimators.

Source specification is flexible in MCNP. The specification of a source particle consists of geometry-location, angular description, energy, time, and particle weight. In addition, probability distributions can exist for any of these variables. Considerable detail is possible in describing a neutron or gamma-ray source or both.

One of the advantages of the MCNP code is that neutron data are processed in a continuous energy sense. The cross sections are read into the code in considerable detail in an attempt to use the information with no significant approximations or distortions. Pointwise neutron cross sections are provided at discrete energies and are tabulated in the Monte Carlo library on an energy grid that is tailored to each isotope. Linear interpolation is used between energy points, with a few hundred to several thousand points typically required. Cross sections are added at a sufficient number of points to insure that the ?inear interpolation constraint reproduces the original cross section tabulation within a specified tolerance. Furthermore, the cross sections are unionized so that ail reactions are given the same energy grid.

All reactions given in a particular cross-section evaluation (such as ENDL or ENDF/B) are taken into account. A choice of three sources of cross sections for most isotopes is available: ENDF/B-IV, ENDL from LLL, and the British (AWRE) library. Resonance parameters, if they are given in the 
evaluation, are processed at several temperatures and the resulting resonance cross sections are added to the pointwise cross sections.

Data for the energy distribution of secondary neutrons are used directly in terms of the "laws" prescribed in the particular cross-section evaluation. Angular distributions for elastic and inelastic scattering events are stored in the Monte Carlo library for 32 equally probable bins on a fine grid of incident neutron energies.

The MCNP code includes a thermalization routine that employs a free-gas mode1. Below a thermal cut-in energy, the lighter atoms are assumed to be in thermal motion, with a Maxwellian distribution of velocities determined by the thermal temperature of the region. Each cell of the problem has specified a unique thermal temperature. Scattering from the light nuclei includes the effect of thermal motion. For nuclei belonging to the heavier groups of atoms and for energies in the thermal range, elastic scattering is assumed to occur in the laboratory system with no energy loss. 


\section{APPENDIX E}

\section{USE OF THE VIRIAL THEOREM AND A SIMPLE SHOCK MODEL TO ESTIMATE} BLAST EFFECTS IN VACUUM AND TWO-PHASE MEDIA

Prior to implementation of program $\mathrm{PAD}^{39}$ for biast mitigation modeling (Sec. III.B.6), two simple approaches to the problem were considered. First, the "virial theorem" 37 provides the simplest model of blast containment in an evacuated vessel. The second approach describes outgoing and reflected shock waves in "mitigating" media using the Hugoniot relations. 48 Both of these techniques are applied here to a 1.13-GJ blast energy described earlier. ${ }^{9}$

1. Virial-Theorem Approach. As a preliminary approach to the blastcontainment problem a convenient baseline for explosive containment is provided by the "virial theorem." ${ }^{37}$ One form of this theorem ${ }^{36}$ predicts that the mass $M$ of a vessel needed to contain a gas or plasma of energy $W$ must satisfy the relationship

$$
M \geq 2 W / f \sigma,
$$

Where $\rho$ is the density of the contairment vessel, $f$ is the number of stress components in the vessel wall ( $f=2$ for a spherical vessel of radius $R$ and thickness $\Delta R$ ), and $\sigma$ is the minimum stress. Taking $M=4 \pi R^{2} \Delta R \rho$ and $f=2$, Eq. (E-1) becomes

$$
R \Delta R \geq(W / R) / 4 \pi \sigma \quad
$$

The relationship between tangential stress $\sigma$ and strain $c$ for thin-walled spheres, $\Delta R<<R$, is given by ${ }^{38}$

$$
\sigma=E \varepsilon /(T-\nu),
$$

where $v$ is Poisson's ratio, and $E$ is Young's modulus. Substituting Eq. (E-3) into $E q$. (E-2) gives the following expressions for the virial theorem if $\varepsilon$ is expressed as microstrain 


$$
\begin{aligned}
(\Delta R / R) \varepsilon & \geq(1-\nu)\left(W / R^{3}\right) 10^{6} / 4 \pi \varepsilon \\
& \geq 2.93(10)^{-7}\left(W / R^{3}\right) \\
& \geq 1.85\left(M_{H E} / R^{3}\right)
\end{aligned}
$$

where $v=0.3, E=1.9(10)^{11} \mathrm{~Pa}\left(28(10)^{6} \mathrm{psi}\right)$, and $M_{H E}$ has the units of $\mathrm{kg}$-equivalent high explosive (HE, 1.5 times the TNT equivalent, $4.2 \mathrm{MJ} / \mathrm{kg}$ ). Equation (E-4) is compared to experimental data 35 in Fig. E-1; these data were obtained at the inception of failure of spherical, steel vessels that were subjected to gradually increased high-explosive charges up to $\sim 20-\mathrm{kg}$ mass. As seen from Fig. E-1, the presence of blast-mitigating or shocktransmitting material within the vessel has a significant effect on the vesse 1 response. The virial theorem shows good agreement with the vacuum case, as is expected; the presence of air or other fluid media leads to shock formation, whereas the pulverization of vermiculite gives an important dissipative channel for blast energy.

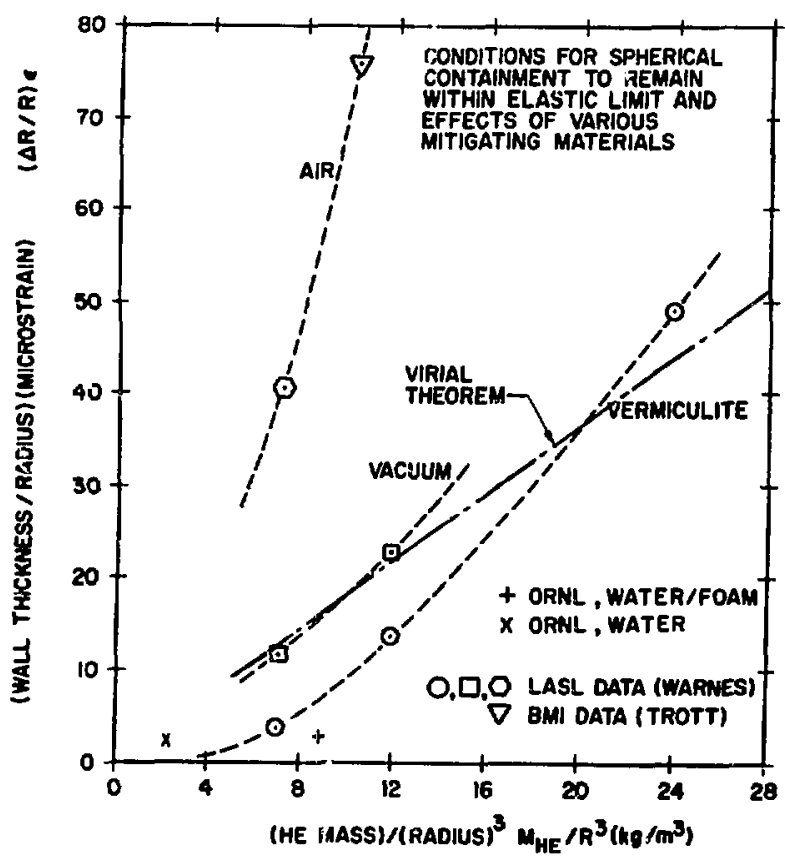

Fig. E-7. Virial-theorem scaling of blast-confinement data using high-explosive (HE) detonations with spherical vessels. All data represent tests which measured only the onset of plastic deformation of the blast-containment vessel. ORNL (Ref. 33), LASL (Ref. 35), BMI (Ref. 49). 
As seen from the analyses in Secs. III.A.4 and III.B.6, energy releases equivalent to $\sim 350 \mathrm{~kg}$ of TNT $(\sim 230 \mathrm{~kg} \mathrm{hiE})$ are expected for recirculating power fractions $\sim 0.25$. Taking $R=2.5 \mathrm{~m}, \mathrm{M}_{\mathrm{HE}}=230 \mathrm{~kg}$, and $\varepsilon=3000$ (failure limit for the steel vessels considered in Fig. E-1, failure generally indicated by beginning of plastic deformation), the required single-shot vessel thickness would be $\sim 20-25 \mathrm{~mm}$ for a "vacuum" or "vermiculite" response.

2. Cyclic Fatigue Constraints. Although some of the data points on Fig. E-1 represent as many as 10 detonations of increasing magnitude, the vessels were always exposed to blast intensities that were sufficiently close to the faiiure threshold to preclude a serious investigation of many-cycle fatigue limits. Fatigue limits should dramatically alter the "single-shot" predictions given above.

The microstrain $\varepsilon=3000$ selected for the above evaluation of $\Delta R$ for $R \sim 2.5 \mathrm{~m}$ generally assures the plastic limit is not exceeded, but this microstrain is too large from the viewpoint of cyclic fatigue. The microstrain must be determined from the fatigue characteristics and desired fatigue life of the containment vessel. Coffin ${ }^{40}$ has correlated the plastic strain $\Delta \varepsilon_{p}$ and elastic strain $\Delta \varepsilon_{e}$ with material properties and the number of cycles to failure $N_{f}$ according to

$$
\begin{aligned}
& \Delta \varepsilon_{p}=C_{2} \nu_{c}^{B(I-k)} / N_{f}^{\beta} \\
& \Delta \varepsilon_{e}=\left(A^{\prime} / E\right) \nu_{c}^{k^{\prime} / N_{f} B^{\prime},},
\end{aligned}
$$

where $\varepsilon \approx \Delta \varepsilon_{p}+\Delta \varepsilon_{e}$, and the constraints for 304 stainless steel at $800 \mathrm{~K}$ and $923 \mathrm{~K}$ are summarized in Table E-I. The last two entries in Table E-I are the microstrains evaluated at the respective temperatures for failure after one year $\left(N_{f}=2.5(10)^{6}\right)$ and ten years of operation for ${ }_{c}{ }_{c}=10 \mathrm{~s}$ $\left(v_{c}=6 \mathrm{~min}^{-1}\right)$ and an $80 \% \mathrm{plant}$ factor. Taking the $800 \mathrm{~K}$ values, based upon corrosion limits, a ten-year lifetime would require $\Delta R \geq 75 \mathrm{~mm}$ for the above FLR conditions ( $R=2.5 \mathrm{~m}, M_{H E}=230 \mathrm{~kg}$ ).

The use of the virial theorem in conjunction with an idealized spherical geometry provides a lower limit for tine blast-confinement problem, although the agreement with the experifriental "vacuum" data on Fig. E-1 lends confidence to this approach. Consideration of the vessei geometry anticipated for a real 
TABLE E-I

SUMMARY PARAMETERS USED TO FIT ANALYTICALLY 304 STAINLESS STEEL FATIGUE DATA, ${ }^{40}$ EQS. (E-5) AND (E-6)

\begin{tabular}{cll} 
CONSTANT & \multicolumn{1}{c}{$800 \mathrm{~K}$} & \multicolumn{1}{c}{$900 \mathrm{~K}$} \\
\hline $\mathrm{C}_{2}$ & $0.300(10)^{6}$ & $1.108(10)^{6}$ \\
& 0.410 & 0.707 \\
$\mathrm{k}$ & 0.93 & 0.81 \\
$\mathrm{~A}^{\prime}$ & $5.29(10)^{11}$ & $2.26(10)^{11}$ \\
$\mathrm{E}$ & $23.4(10)^{6}$ & $21.6(10)^{6}$ \\
$\mathrm{~K}^{\prime}$ & -0.02 & 0.089 \\
& 0.20 & 0.187 \\
$\mathrm{~N}_{\mathrm{f}}=2.5(10)^{6}$ & 1898 & 823 \\
$\mathrm{~N}_{\mathrm{f}}=2.5(10)^{7}$ & 1016 & 516
\end{tabular}

engineering structure (i.e., stress concentration points, penetrations, acoustical responses, etc.) in conjunction with the formation of shocks will undoubtedly lead to larger vessel dimensions. The effect of shock generation in an intervening medium is examined approximately in the following section. Generally, however, the vessel dimensions based on the predictions of the virial theorem should be viewed as iower limits, and a detailed structural design is required before the containment problem can be further quantified.

3. Blast Confinement in a LiPb/He Bath. The original conceptualization $^{5}$ of the FLR envisaged the use of a He-bubble-impregnated LiPb bath to attenuate the post-implosion biast. This system is shown schematically in Fig. III-23. Also shown are systems which operate in vacuum ard in a fluidized bed of blast-mitigating material. For the former case the blanket must surround the vacuum vessel, whereas the fluidized bed might contain a lithium-iearing oxide with shock-mitigating properties similar to vermiculite (Fig. E-1). Only the LiPb/He containment scheme, which, as will be shown at best will respond according to the virial-theorem predictions (Sec. $\Sigma-1)$, is addressed here. 
3.1. Development of Shock Model. A simple model was developed to consider spherical shocks in liquid-gas mixtures. Specifically, a lead-lithium mixture is considered for the primary coolant and tritium breeding, and helium bubbles are used for shock mitigation. Dresner ${ }^{32}$ has suggested that shock riitigation would be enhanced by creating helium bubbles in the liquid metal.

The lead-lithium mixture is treated as an incompressible fluid and the helium as an ideal gas. Initially the helium occupies a fraction $f_{H e}$ of the mixture volume. For $f_{H e} \ll 1$ the helium is simply considered as a fine dispersion of bubbies, and for $f_{H e} \leq 1$ the lead-lithium is assumed to be in the form of a shower or mist of droplets. This latter case is treated in Sec. III.B.6.

The 14.1-MeV neutron heating will form a substantial shock in pure lithium for the fusion yields considered here, bu a small fraction of helium bubbles should easily mitigate that shock. ${ }^{32}$ Most of this neutror energy heats the liquid-metal coolant/breeder. When bubbles are present, the thermal expansion of the liquid metal is easily taken up by the bubbles with little accompanying pressure-volume work, and most of the neutron energy remains as thermal energy in the lithium breeder. The post-burn energy which remains in the piasma and vapurized liner debris is of primary corcern. The decompression of hot gas and plasma can perform far more work than a corresponding decompression of the neutron-heated coolant.

An energy $W \sim\left(W_{L}+W_{\alpha}\right)$ is assumed to heat an ideal gas or plasma of radius $r_{10}$ equal to the initial liner radius. For the $l=0.2-m-l o n g$ liner this explosive energy is $\sim 1.0 \mathrm{GJ}$. For this computation $r_{10}$ is taken to be $0.20 \mathrm{~m}$. An adiabatic expansion of the plasma is assumed. Setting the specific-heat ratio $\gamma=5 / 3$ for this hot gas and defining $r_{j}$ as the time-dependent inner radius of the post-implosion cavity created in the liquid-metal, the plasma pressure $P_{i}$ as a function of $r_{j}$ becomes

$$
P_{i}=P_{0}\left(r_{10} / r_{i}\right)^{5}, P_{0}=W / 2 \pi r_{10}^{3} \text {. }
$$

It is further assumed that a single shock travels from $r_{10}$ to the radius of the vessel wall, $R$, where a second shock is formed and returns to the plasma/liquid-metal interface. During this iniard motion of the reflected shock the highest pressures on the vessel wall would occur. In order to model the shock motion, the following definitions are made. 
$r_{s}=$ radial position of shock $(m)$

$u_{s}=$ radial velocity of shock $(m)$

$u_{p}=$ fluid velocity behind shock $(\mathrm{m} / \mathrm{s})$

$P_{a}=$ ambient pressure of LiPb-He mixture $(\mathrm{Pa})$

$V_{L}=$ specific volume of liquid $L i P b=1 / \rho_{L_{3}}\left(\mathrm{~m}^{3} / \mathrm{kg}\right)$

$V_{\mathrm{a}}=$ specific volume of ambient $\mathrm{LiPb}-\mathrm{He}\left(\mathrm{m}^{-3} / \mathrm{kg}\right)$

$\mathrm{f}_{\mathrm{He}}=$ initial helium fraction $=1-V_{\mathrm{L}} / \mathrm{V}_{\mathrm{a}}$

$P_{S}=$ pressure directly behind shock $(\mathrm{Pa})$

$V_{s}=$ specific volume behind shock $\left(\mathrm{m}^{3} / \mathrm{kg}\right)$

$E_{a}=$ ambient specific energy $(\mathrm{J} / \mathrm{kg})$

$E_{S}=$ specific energy behind shock $(\mathrm{J} / \mathrm{kg})$

Figure E-2 depicts the geometry and associated notation.

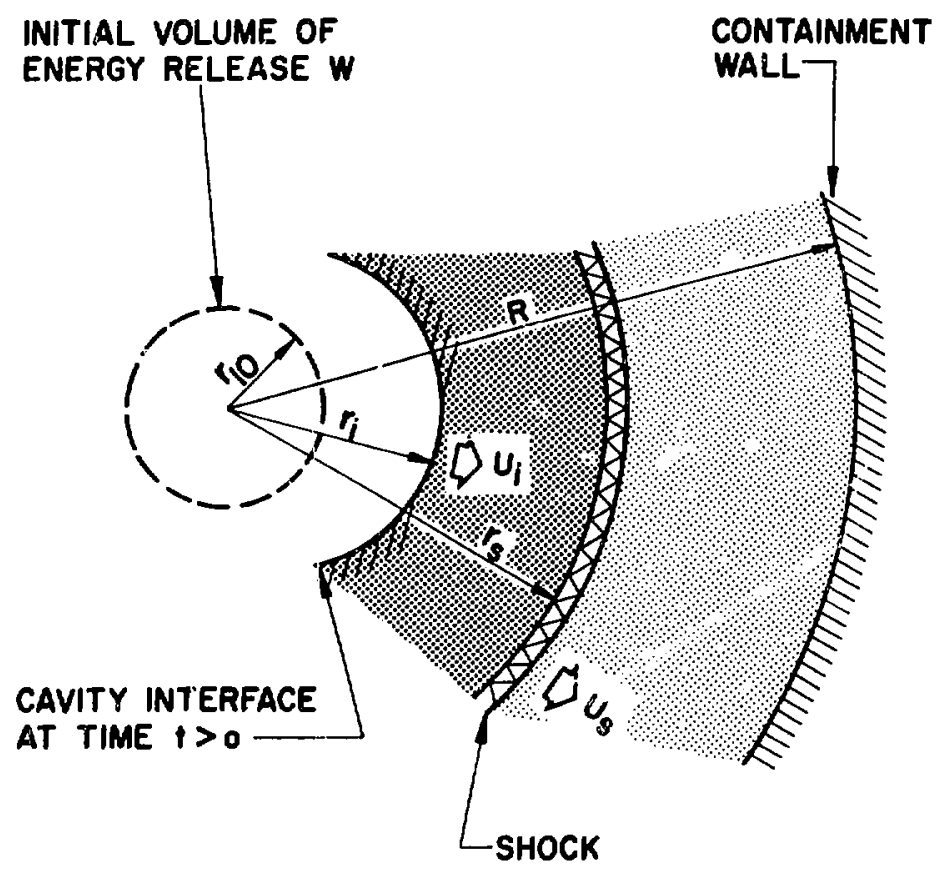

Fig. E-2. Schematic diagram of simple shock model used to describe the pressure loading of a thin spherical containment shell subjected to the reflection of a coherent shock generated in a liquid/gas mixture. Refer to cext for notation. 
Conservation of energy and momentum are used to derive the Hugoniot equations ${ }^{48}$ that relate pressure, volume, energy, and velocity on one side of the shock to similar quantities on the other side

$$
\begin{aligned}
& u_{s}=v_{a} \sqrt{\left(P_{s}-P_{a}\right) /\left(V_{a}-v_{s}\right)} \\
& u_{p}=u_{s}\left(1-v_{s} / V_{a}\right) \\
& E_{s}-E_{a}=\left(P_{a}+P_{s}\right)\left(v_{a}-v_{s}\right) / 2
\end{aligned}
$$

An equation of state (EOS) completes the relationship between properties on each side of this shock. Two EOS models are used; the first requires that the total increase in specific energy across the shock heats the helium bubbles

$$
P_{s} V_{s}^{Y}=P_{a} V_{a}^{Y}(Y=5 / 3)
$$

The second EOS model assumes that the shock heats the liquid metal, and the helium bubbles are adiabatically compressed

$$
E_{s}-E_{a}=(3 / 2)\left[P_{s}\left(V_{s}-V_{L}\right)-P_{a}\left(V_{a}-V_{L}\right)\right] \text {. }
$$

These two models represent significantly different physics and will be discussed later.

To complete the equation of motion for the shock, the equations for acce 1eration and conservation of mass are introduced.

$$
\begin{aligned}
& \rho_{s}\left(d u_{p} / d t\right)+\nabla p_{s}=0 \text { and } \\
& \Delta \cdot\left(\rho u_{p}\right)+\partial \rho / \partial t=0 .
\end{aligned}
$$

At this point the simplifying ssumption is made that once a volume element is compressed by passage of the shock the specific volume, $V_{s}$, does not change thereafter (i.e., $\left.d \rho / d t=d V_{s} / d t=0\right)$. This assumption enables Eq. (E-14) to be replaced with the relation

$$
r^{2} u_{p}=r^{\prime 2} u_{p}^{\prime}
$$


where $r$ and $r^{\prime}$ represent any two points behind the shock. Since fluid velocities and accelerations at all points are now related to one point (e.g., at the shock), Eq. (E-13) can be integrated over radius to yield an ordinary differential equation rather than a partial differential equation; this assumption greatly simplifies the numerical solution.

Undoubtedly a number of shortcomings and inconsistencies can be found with the assumption that $d \rho_{s} / d t=0$ after passage of the shock. For instance, the resulting model does not apply to shocks in purely gaseous media, where compressed gas behind a shock would expand as the driving pressure decreases (Eq. (E-7)). When a liquid-gas mixture is shocked, such an expansion will certainly be reduced if not reversed. The hot, compressed gas would lose heat to the liquid and be less able to expand as described above.

Defining the following quantities

$$
\begin{aligned}
& G=r_{s} \int_{r_{i}}^{r_{s}}\left(\rho_{s} / r_{s}^{2}\right) d r \quad \text { and } \\
& H=r_{s}^{4} \int_{r_{i}}^{r_{s}}\left(\rho_{s} / r_{s}^{5}\right) d r,
\end{aligned}
$$

and combining Eqs. (E-8), (E-9), (E-10), (E-13), (E-16), and (E-17) results in the following expression for the particle velocity $u_{p}$.

$$
d u_{p} / d t=-\left[\left(P_{s}-P_{j}\right) / G+2 u_{p}^{2}\left(1-H / G-1 /\left(1-v_{a} / v_{s}\right)\right)\right] / r_{S}
$$

A computer code was written to combine Eqs. (E-7), (E-8), (E-9), (E-10), $(E-11)$, and $(E-18)$ and to solve for $r_{s}(t)$.

The description of the reflected shock is greatly simplified here to give an average pressure during its reverse transit across the fluid. This mode 1 is coupled with the appropriate EOS (Eq. (E-11)) to solve for an average pressure during reflection, $\bar{P}_{r}$. The quantity $i_{r a}$ is defined as the time for the outgoing shock to impact the vessel wall and $t_{r b}$ as the time the reflected shock reaches the inner surface of the fluid. The average specific volume of the reflected shock is $V_{r}=4 / 3 \pi\left[R^{3}-r^{3}\left(t_{r b}\right)\right] / M_{L}$ where $M_{L}$ is the total fluid mass. It is easily shown that

$$
\bar{p}_{r} \simeq \frac{u_{p}\left(t_{r a}\right)\left[R^{2} / r^{2}\left(t_{r b}\right)-1\right]}{v_{r}\left(t_{r b}-t_{r a}\right)} \text {. }
$$


Equation (E-19) is combined with Eq. (E-11) or Eq. (E-12) to solve for $\bar{p}_{r}$.

3.2. Computational Results. The results of several computations are shown in Fig. E-3. The tension in the vessel wall, $T=\bar{P}_{r} / R$ is compared to the virial-theorem result (Sec. E. 1), $T_{v}=W / 2 \pi R^{2}$. The ratio $T / T_{v}$ is equal to the ratio of respective tangential stresses $\sigma / \sigma_{v}$ and is given in Fig. E-3 as a function of the helium fraction $f_{H e}$ for the following conditions:

$$
\begin{aligned}
& W=1.13 \mathrm{GJ} \\
& \rho_{L}=9400 \mathrm{~kg} / \mathrm{m}^{3} \\
& R=2.3 \mathrm{~m} \\
& r_{10}=0.2 \mathrm{~m} \text { and } 2 \mathrm{~m}
\end{aligned}
$$

The two EOS (Eqs. (E-11) and (E-12)) models give surprisingly similar results, as shown in Fig. E-3. A shock-heated gas is compressed to no less than $25 \%$ of its original volume; however, a much greater compression occurs when a portion of the shock heat is also delivered to the liquid metal. Typically the shocked helium would then occupy only a few percent of its original volume. Even with this significant difference the results agree to within an order of magnitude for any given value of $f_{\mathrm{He}}$ and $r_{10}=0.2 \mathrm{~m}$.

The results given here do not show a stress reduction such as that given by vermiculite (Fig. E-1). Although computed results show that shock heating can dissipate over $98 \%$ of the blast energy, sufficient momentum is generated in the liquid metal to produce substantial wall stresses compared to the predictions of the virial theorem. Two complementary phenomena appear to be in effect. When the helium is highly compressed, as for the EOS model of Eq. $(E-12)$, a larger amount of energy is dissipated in the shock. When this more dense mixture (as compared to the EOS model of Eq. (E-11)) strikes the wall, the shock reversal is more sudden because of the smaller second compression that can occur.

The hydrodynamic computations of program PAD (Sec. III.B.6) do not support the predictions of an increase in wall stress, corresponding to the reduction of $\gamma$. The maximum stresses given in Fig. III-19 for a 1.46-G.l blast with $R=2.6 \mathrm{~m}$ were converted in terms of $\sigma / \sigma_{v}$ and incorporated intr; Fig. E-3. 


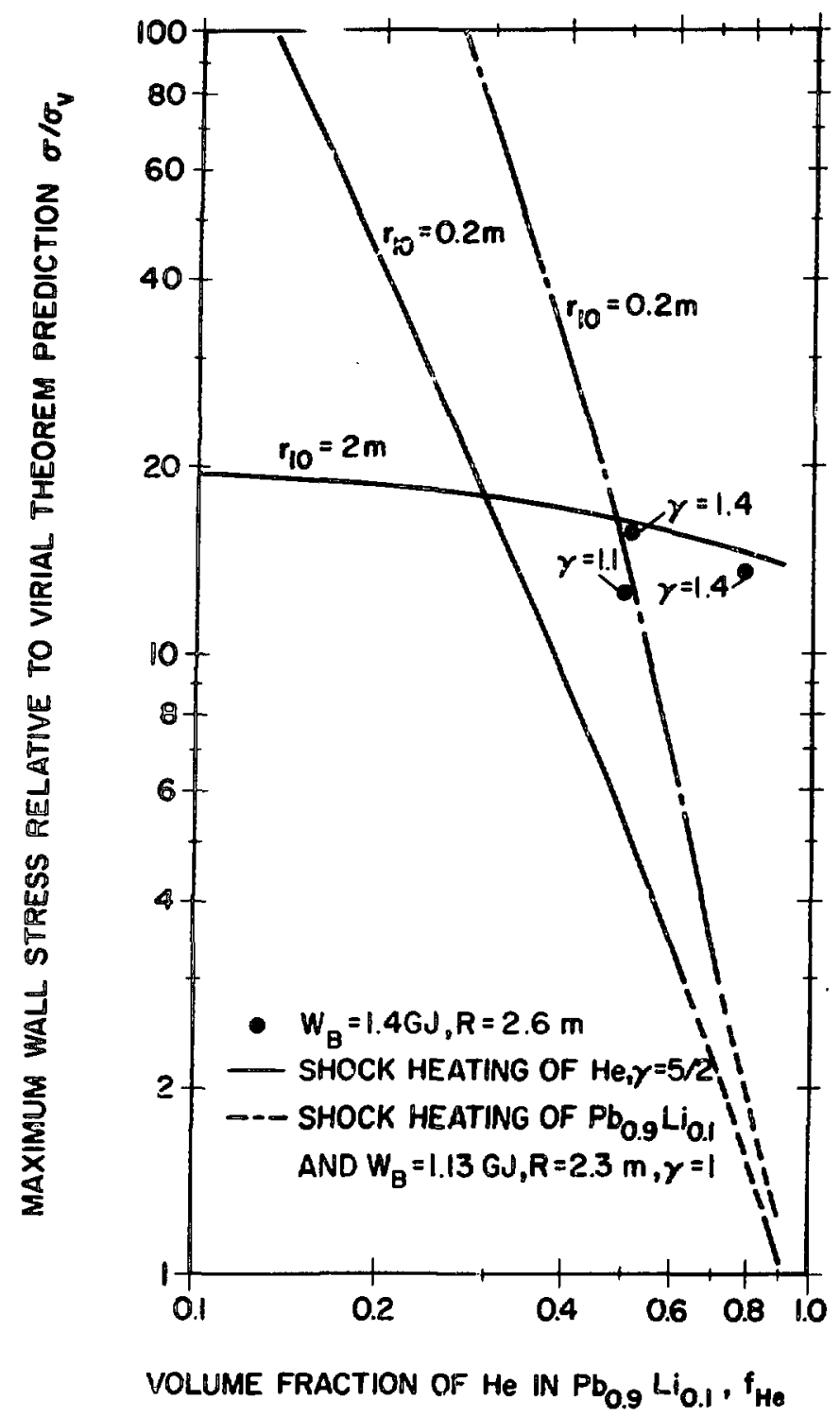

Fig. E-3. Dependence of maximum wall stress relative to predictions of the virial theorem as a function of the bubble void fraction. The energy released at the center of the spherical vessel is $W$, the vessel radius is $R$, and the specific heat ratio of the gas is $\gamma$. Shown also are results for similar conditions from the hydrodynamic code PAD. 39 
Since $W$ and $R$ differ slightly between the two computations, comparison is not entirely justified, but trends are indicated. The PAD results with $\mathrm{f}_{\mathrm{He}}=0.5$ fall close to the $\gamma=1$ curve of the simple shock model; however, stresses increase with $\gamma$ according to the PAD model, rather than decrease. Also, the PAD results do not show the sharp decrease in $\sigma, \sigma v$ as $f_{H e}$ approacines unity, as illustrated by the $f_{H e}=0.8, \gamma=1.4$ point on Fig. E-3. Most of the discrepancies seen here probabiy arise because the simple shock model does not allow for expansion of shocked gas (i.e., $\mathrm{d} \rho_{\mathrm{s}} / \mathrm{dt}=0$ ).

Serious shortcomings of the simple models upon which Fig. E-3 is based are:

(a) The simple shock approach toes not allow for expansion of a shocked gas (i.e., d $\rho_{s} / d t=0$ ).

(b) Program PAD allows post-shock expansion according to an ideal gas law. In fact the liquid would be heated by irreversible processes, reducing expansion behind the shock to a level between the PAD and simple shock models.

(c) Although PAD incorporates an artificial viscosity to affect a shock, no detailed empirical knowledge of shocked gas-fluid mixtures is used. It may be necessary to wait for experimental results to improve this part of the model.

4. Conclusion. For $1.13 \mathrm{GJ}$ of explosive energy released by a liner, the virial theorem predicts for a containment vessel radius $R=2.0 \mathrm{~m}$ that the wall thickness $\Delta R \simeq 28 \mathrm{~mm}$ based upon a "single-shot" criterion (microstrain $\varepsilon=3000$ ). Consideration of cyclic fatigue constraints (for 304 stainless stee1) leads to a 2.5-m-radius vessel with $\Delta R=75-\mathrm{mm}$ wall thickness $\left(\tau_{c}=10\right.$-s cycle time for a 10 -year life at an $80 \%$ plant factor). The virial theorem predicts surprisingly well experimental data from vacuum detonations in spherical steel vessels. Using the virial theorem to scale experimental data from detonations in air-filled vessels results in significantly increased vessel wall thicknesses presumably because of momentum amplification by shock propagation in the gaseous medium.

A simple shock-propagation model was deveioped to investigate the shock mitigation properties of He-bubble containing lead-lithium liquid alloy. No reasonable bubble fraction could be found which resulted in containment-vessel wall stresses that are below the predictions of the virial theorem (vacuum medium); the acceleration of the lead-lithium mass causes significant pressure amplification for all He-bubble fractions considered and for two extreme EOS models used to describe the two-phase system. 
A one-dimensional hydrodynamic code, PAD, ${ }^{39}$ was used to model gas-liquid mitigators more carefully. Good agreement was seen between PAD and the simple shock model ior equal initial volume fractions of gas and liquid, but wide discrepancies occur for small liquid fractions. Until more complete theoretical and/or empirical data are available, the most reliabie results are for equal liquid-gas mixtures. 


\section{APPENDIX $F$ \\ COSTING GUIDELINES, ACCOUNTING SYSTEM, AND DATA BASE}

The comparison of the economic merits of one fusion concept with another can be made only if the basic cost estimates are performed on a uniform and normalized basis. Although the DOE/OFE is in the process of generating such a normalized basis, ${ }^{41,42}$ the required information is not complete and available to the fusion community. This study has adopted these procedures as they exist in interim form ${ }^{4,42}$ and when necessary has provided the missing components, again on an interim basis, in order that a complete cost estimation of the FLR concept could be completed within FY 1978. Presented here is a summary of the costing guidelines ${ }^{41}$ as they exister in early 1978; the cost accounting system and the cost data base used by this study are also included.

Table F-I summarizes the costing guidelines, whereas Table F-II sumarizes the cost data bases that have been assembled from a number of sources indicated. If " 0 " is entered into the "unit cost" column in Table F-II, the cost of this item has been agglomerated into a higher level cost. If a "l" is entered into the "number of units" column, this item is acknowledged, but has been taken into account at a higher level. If a " 0 " is entered instead, that item does not exist or does not pertain to the concept. If the entry is other than " 0 " or " 1, " the number of units is specified. A "- 1 " entered into this column indicates a fractional unit is required, but usually its cost is taken into account at a higher level. Last, Table F-III presents a detailed cost breakdown upon which Table III-III in Sec. III.D.2 is based. 


\section{SUMMARY OF GUIDELINES USED IN COMMON COSTING PROCECURE $41,4 \hat{\imath}$}

- Although a maximum, practical plant size of 5000 MWe is established, the actual plant size and associated number of units per power plant is established on the basis of specific cost optimization. In this context, approximately 8 FLR units were selected to give a net electrical ouput of 21000 MWe, primarily because of turbine and steam-generator costs.

- The costing of design and engineering activities assumes the existence of a mature industry for all major reactor and balance-of-plant components.

- All labor, materials, equipment-during-construction, plant startup, and plant operating costs are based on January 1, 1978 do 11 ar values.

- The costing methodology is based upon similar schemes used by investorowned (private) utilities rather than for a public utility project.

- The capital cost accounts are given in Table F-II and are composed of direct, indirect, and time-related costs.

-- Direct costs are determined by the best estimates of component costs on the basis of a detailed, well-documented conceptual design.

-- Indirect costs are determined as a percentage of the direct costs: 15\% for construction facilities, equipment, and services; $5 \%$ for taxes, insurance, and plant startup; 15\% for engineering and management.

-- Time-related costs are composed of only interest during construction. Although riumerous methods exist for computing the time-related costs, 50 the particular method selected here applies for an integrated cash flow that is skewed towards the back end of the construction period, leading to a half-cash-flow at $60 \%$ of the construction period. Hence, escalation and interest are computed as a percentage of the direct plus indirect costs assuming a 10-yr construction period; aggregate percentages of $33.8 \%$ and $64.4 \%$, respectively, result for an escalation rate of $5 \%$ and an interest ratio of $10 \%$.

- Operating and maintenance costs reflect the daily, routine expenditures incurred during plant operation and are specified in detail by Ref. 41. Nuclear 1 ability insurance, licenses and fees, and working capital are not included. General1y, operating and maintenance costs equal $2 \%$ of the total capital (direct plus indirect plus interest during construction). If an exceptional operating and maintenance cost is incurred, such as the leads and liner cost for the FLR, this cost is computed by a separate optimization procedure (Appendix $C$ ) and added to the "normal" operating maintenance costs.

The following assumptions are used to compute the power costs (mills/kWeh)

-- plant power factor is 0.85 
TABLE F-I cont'd.

-- plant operating life is $30 \mathrm{yr}$

-- cost of debt is $10 \%$ per year

-- cost of equity is 15\% per year

-- escalation is 5\% per year 
岕
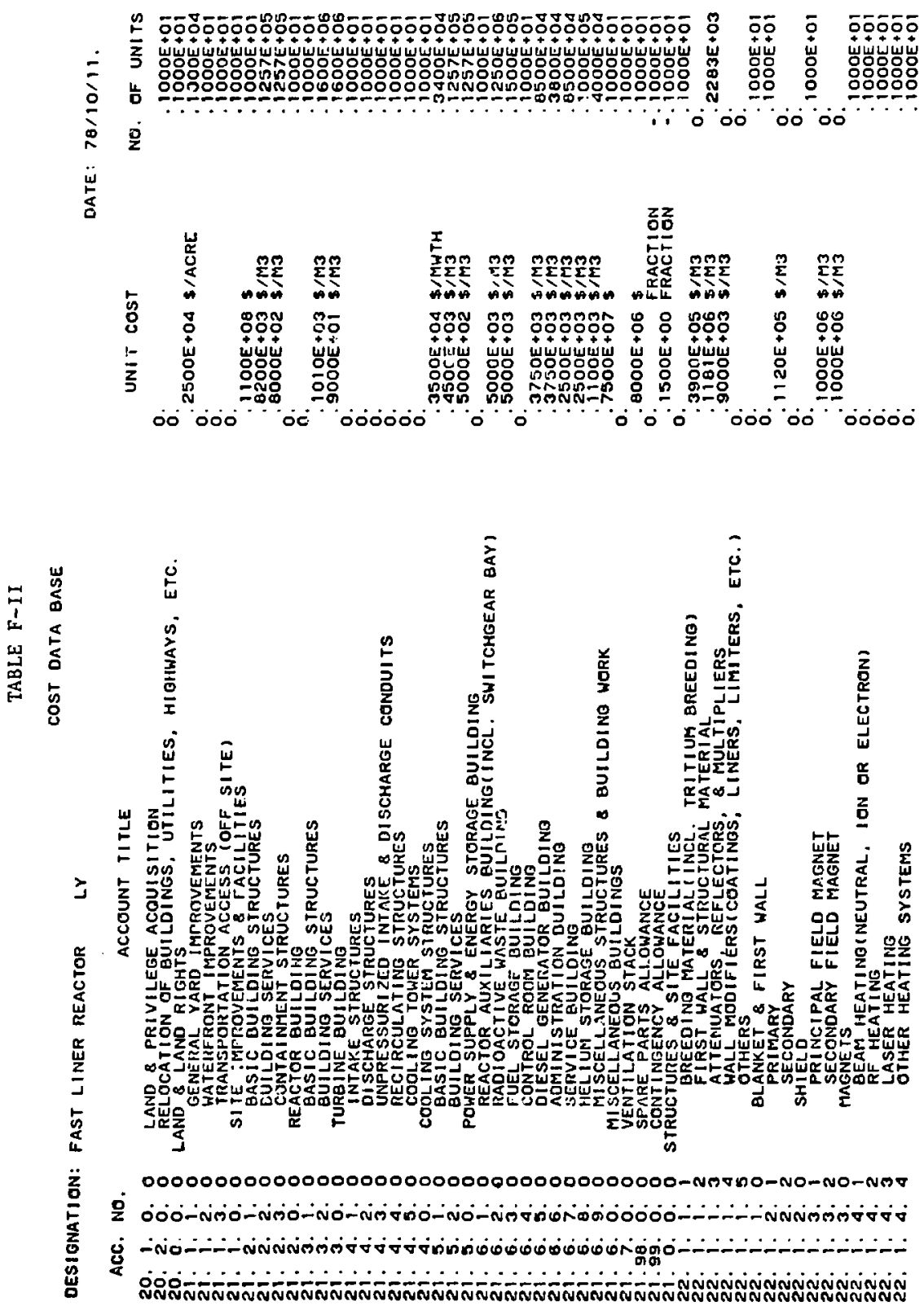

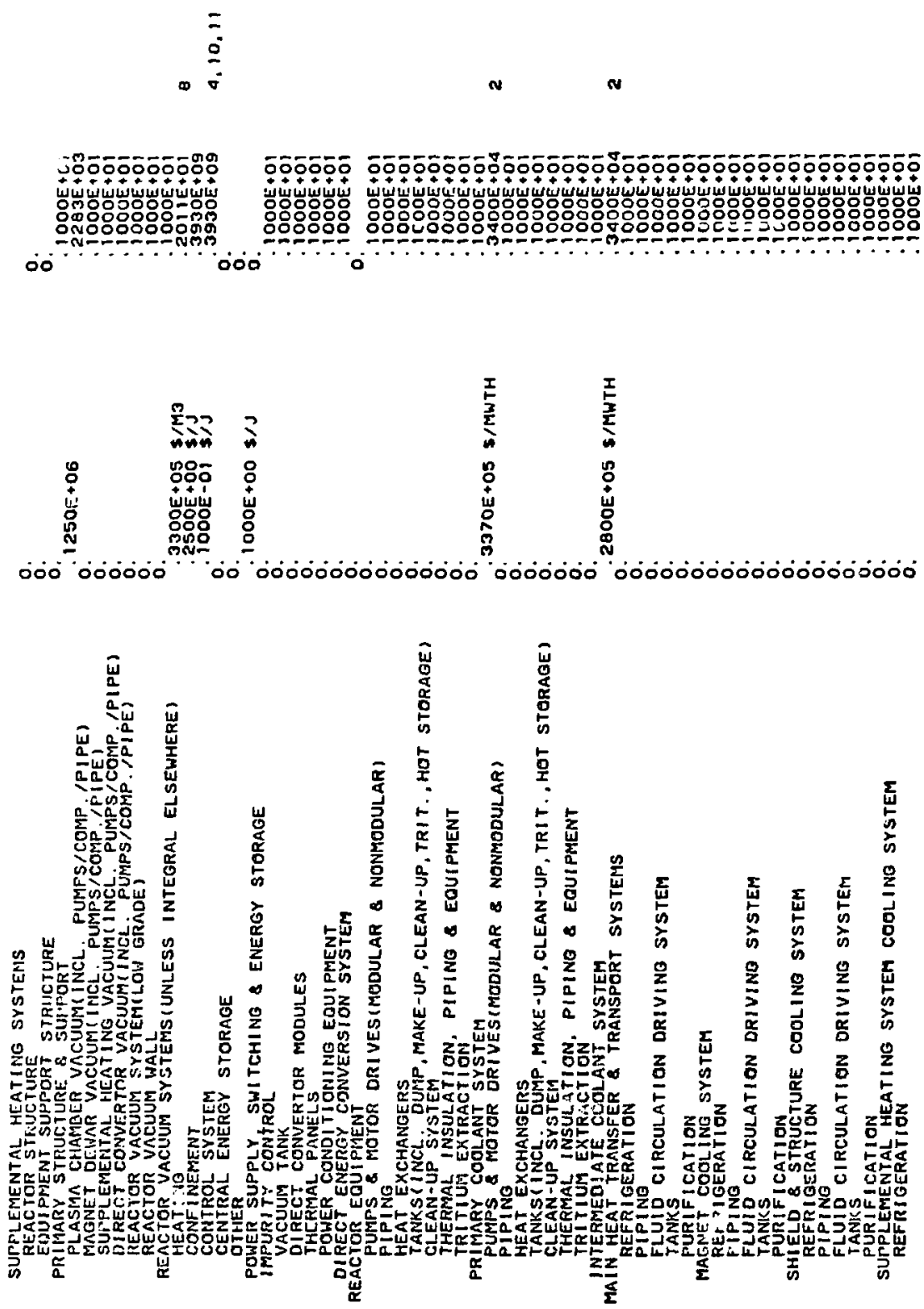

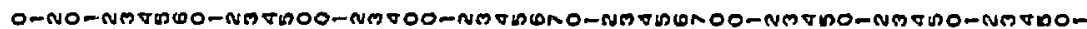
نंvis

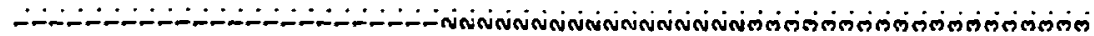

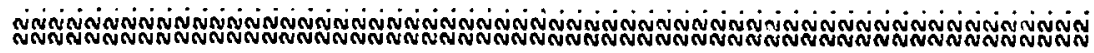



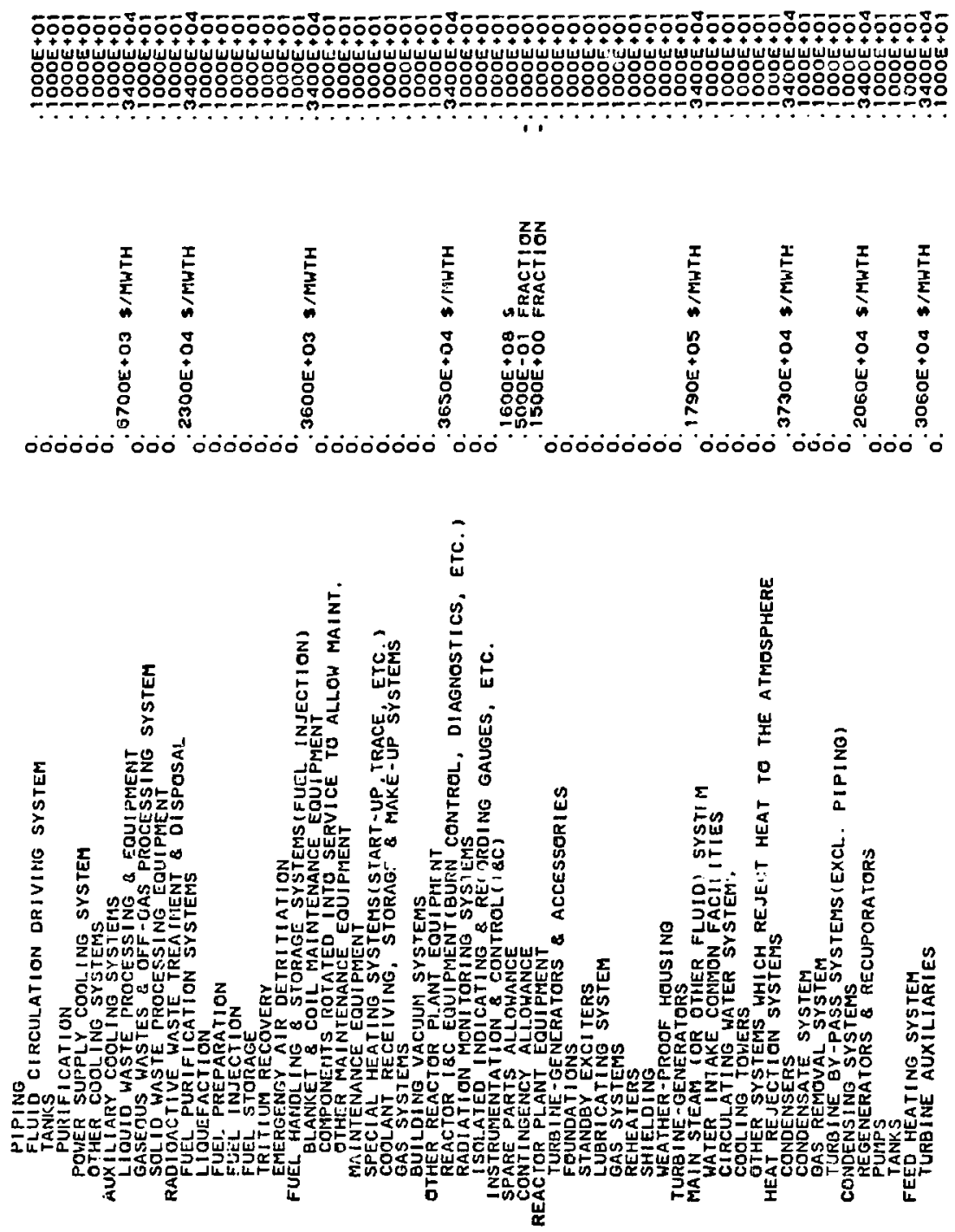

Nevr,000000000000000-NR00000000000000000000000000000000000000

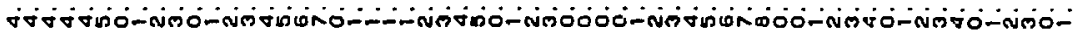

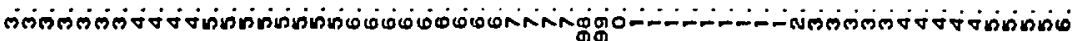

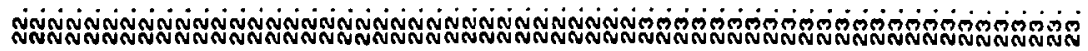





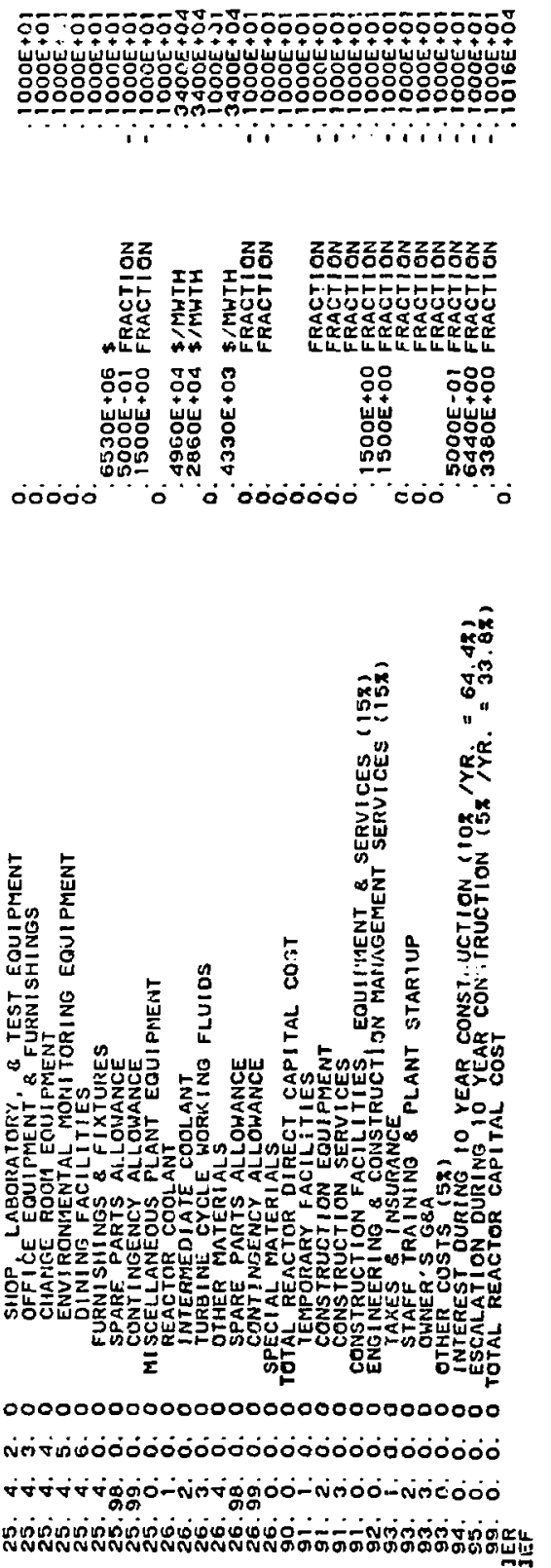
TABLE F-III

FUSION REACTOR ECONOMIC EVALUIATION

DESIBNATION: FAST LINER REACTOR

ACC. NO.

$$
\text { ACCOUNT TITLE }
$$

DATE: $78 / 10 / 11$.

20. 1. LAND \& PRIVILEGE ACOUISITION

20. 2. RELOCATION OF BUILDINGS, UTILITIES. HIGHWAYS, ETC.

20. LAND \& LAND RIGMTS

2: 1: 1. GENERAL YARD IMPROVEMENTS

21: $1:$ 3: TRANSFORTATION ACEESS (OFF SITE)

21: 2. 1: BASIC BUILDING STRUCTURES

21: 2: $3:$ BUILOING SERVICES

21: 3. 1: BASIC BUILDING STRUCTURES
21: 3: $2: \quad$ BUILONMG SERVICES
21: 3.

INTAKE STRUCTURES

\& OISCHARGE CONDUITS

21: $4: \frac{1}{2}:$

21: 4 4: 5 :

21: 5.: $1:$

COOL ING TOWER SYSTEMS
COULING SYSTEM STRUCTURES

BASIC BUILOING STRUCTURES

POWER SUPFLY \& ENERGY STORACE BUILOING

21: 6. 1. REACTIR AUXILIARIES BUILDINOIINCL. SHITCHGEAR BAYI

FUEL STORAGE OUILDING

COHTROL ROOM BUILOING

AOMINISTRATION BUILDING

HELIUM STORAGE QUILOINO
MISCELLANEOUS STRUCTURES \& BUILOINO HORK
MISCELANEOUS BUILDINGS

VENTILATION STACK

SPARE FARTS ALLOWANCE

21.98.

21

STRUCTURES \& SITE FACILITIES
MILLION DOLLARS

\subsection{0}

11.000

10.307

11.313

16.160

17.600

11.900

0.657
.629

6.2es

62.500

3.180
1.425
2.125
2.500
.440

87.178

.800

21.911

167.987 


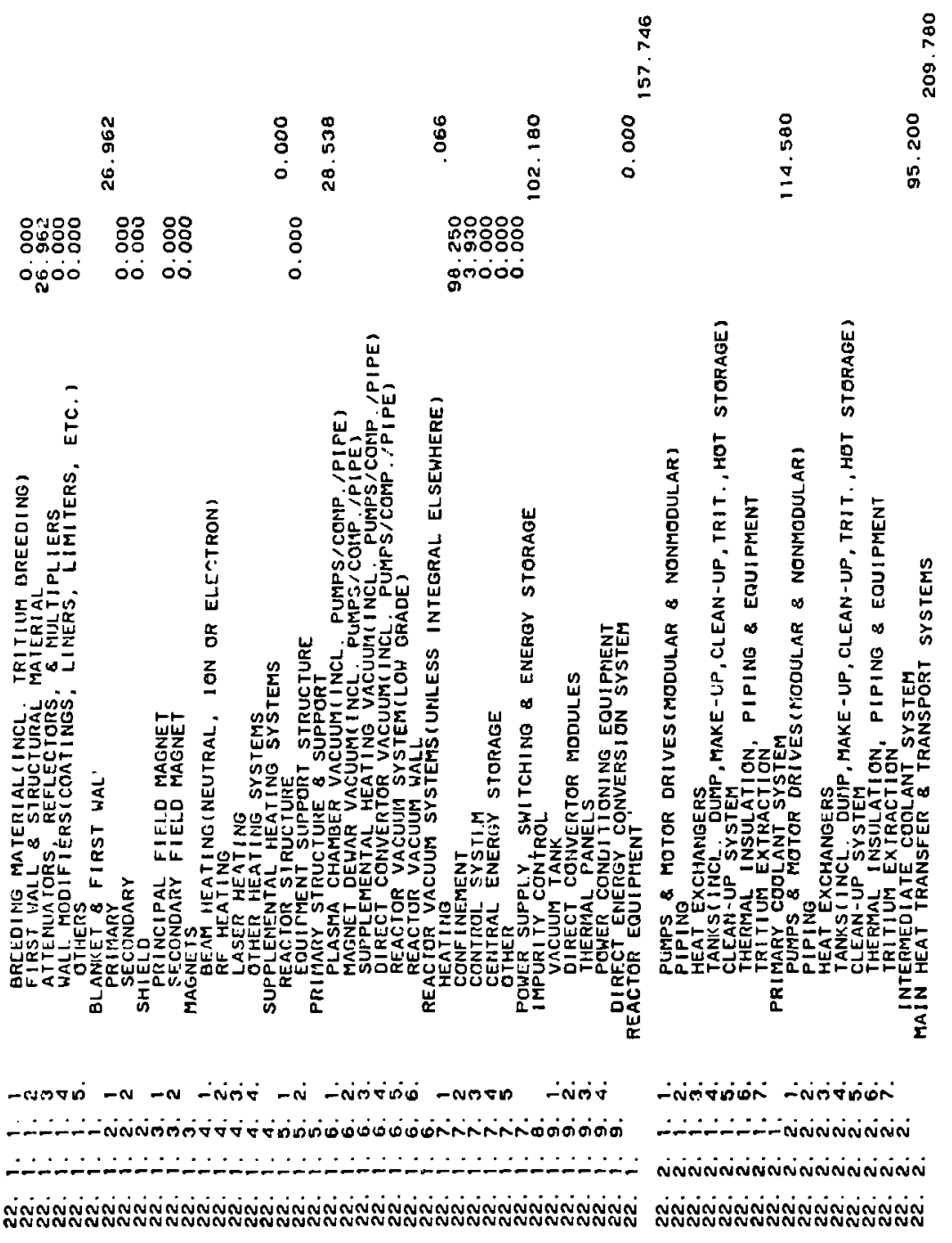




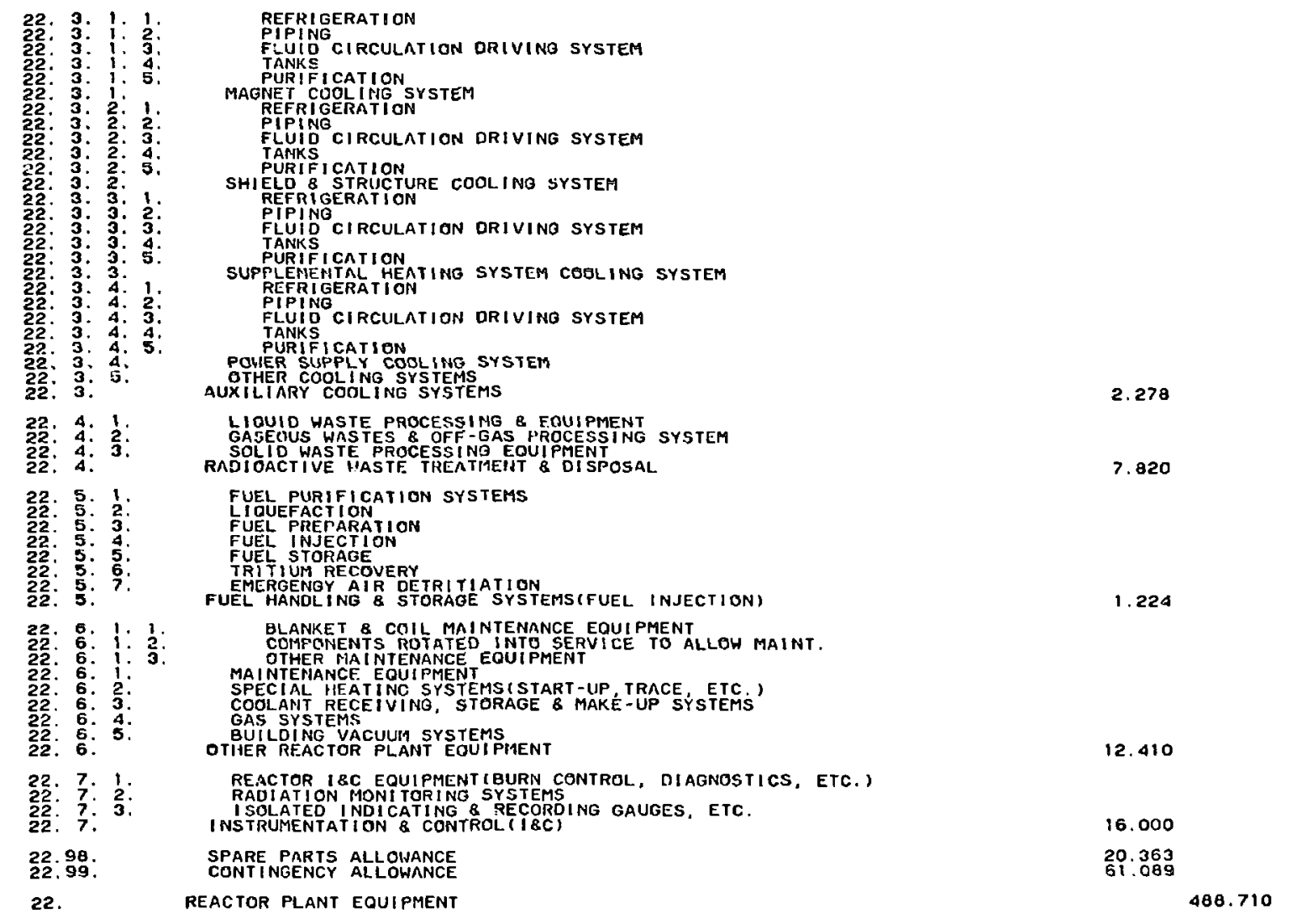




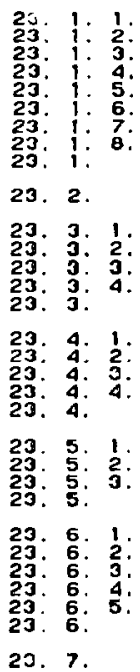

23. 90 .

23. 99 .

23.

TUROINE-OFNERATORS \& ACCESSORIES

FOUMDAT IONS

STANDBY EXCITERS

LUBR SYSTES

REHEATERS

SHIEIERS

WEATHER -PROOF HOUSINO

TURBINE-GENERATORS

00.860

MAIN STEAM (OR OTI:LR FLUID) SYSTEM

WATER INTAKE COHMON FACILITIES

CIRCULATING WATER SYSTEMS

OTHER SYSTEMS HHICH REJECT hEAT TO THE ATMOSPIHERE

12.682

CONDENSERS

CONDENSATE SYSTE.1

GAS REMOVAL SYSIYM

CONDENSING SYSTEMS

REGENERATORS \& NECUPORATORS

PUMPS

FEED HEATING SYSTL'A

10.404

TURB I NE AUXILIAF: ES

AUXILIARAES COOL ING SYSTEM(EXCL. PIPINO)

CHEMICAL TREATMEIT \& CWOEXS ATE PURIFICATION SYSTEMS

CENTRAL LUBRICATION SERVICE SYSTEMIEXCL. PIPINB)

OTHER TURB INE PLAIIT EGUUIMENT

63.920

(NSTRUMENTATION \& CONTROL(IBG) EOUIPMENT

1.972

SPARE PARTS ALLOH NICE

.157

TURE INE PLANT EQUIPIENT

156.999

24. 1: $1 . \quad$ GENFRATOR CIRCUITS
24: 1: $2 . \quad$ SIATION SERVICE

24. 2. 1. STATION SERVICE \& STARTUP TRANSFORMERS

24: 2: 2 : LOW VOLTAGE UNIT SUBSTATION 8 LIGHTING TRANSFORMERS

24. 2. 3.

24. 2 . 5 .

24. 2 . 6

GAS TUR:BINE GE GEMERATORS

MOTOR GFIERATOR SETS

24. 3. 1

STATION SERVICE EOUIPIIENT

MAIN CONTROL BOARD FOR ELECTRIC SYSTEM

SWUXILIARY POHER \& SIGNAL BOAROS

24. 3 :

24. 4. 1

GEN. STATION GROUNDINO SYSTEM \& CATHODIC PROTECTION

24. 5. 1 .

24: 5: 3 :

24: $5: 4$

24. 6. !

24: $6: 3$

24: $5: 4$.

24. 6 .

24: 7: $\frac{1}{2}:$

$24: 7: 3$

24: 7: 5 :

24: 7: $7:$

24. 7 :

24.98.

CONCRETE CABLE TUNNELS. TRENCHES \& ENVELOPES

CASLE T

OTHER 'STRUCTURES

ELECTRICAL STRUCTURES \& WIRING CUNTAINERS

4.760

GENERATOR CIRCUITS WIRING

STATION SERVICE POWER HIRINO

CONTROL WIRINO

INSTRUMENT WIRING

CONTAL NMENT PENETRATIONS

OL WIRINO

9.466

REACTOR BUILOING LIGHTING

REACTOR AUXILIARIES BUILDINO LIGHTINO

RADIOACTIVE WASTE BUILDING I IGHTINO

FUEL STORAGE BUILDING LGHTIHG

MISCELLANEOUS BUILDINGS LIGHTIHG

ELECTRICAL LIGGTING

SPARE PARTS ALLOWANCE

CONTINGENCY ALLUWANCE

9.384

3.126

.109

1.265

ELECTRIC PLANT EOUIPMENT

05. 231 


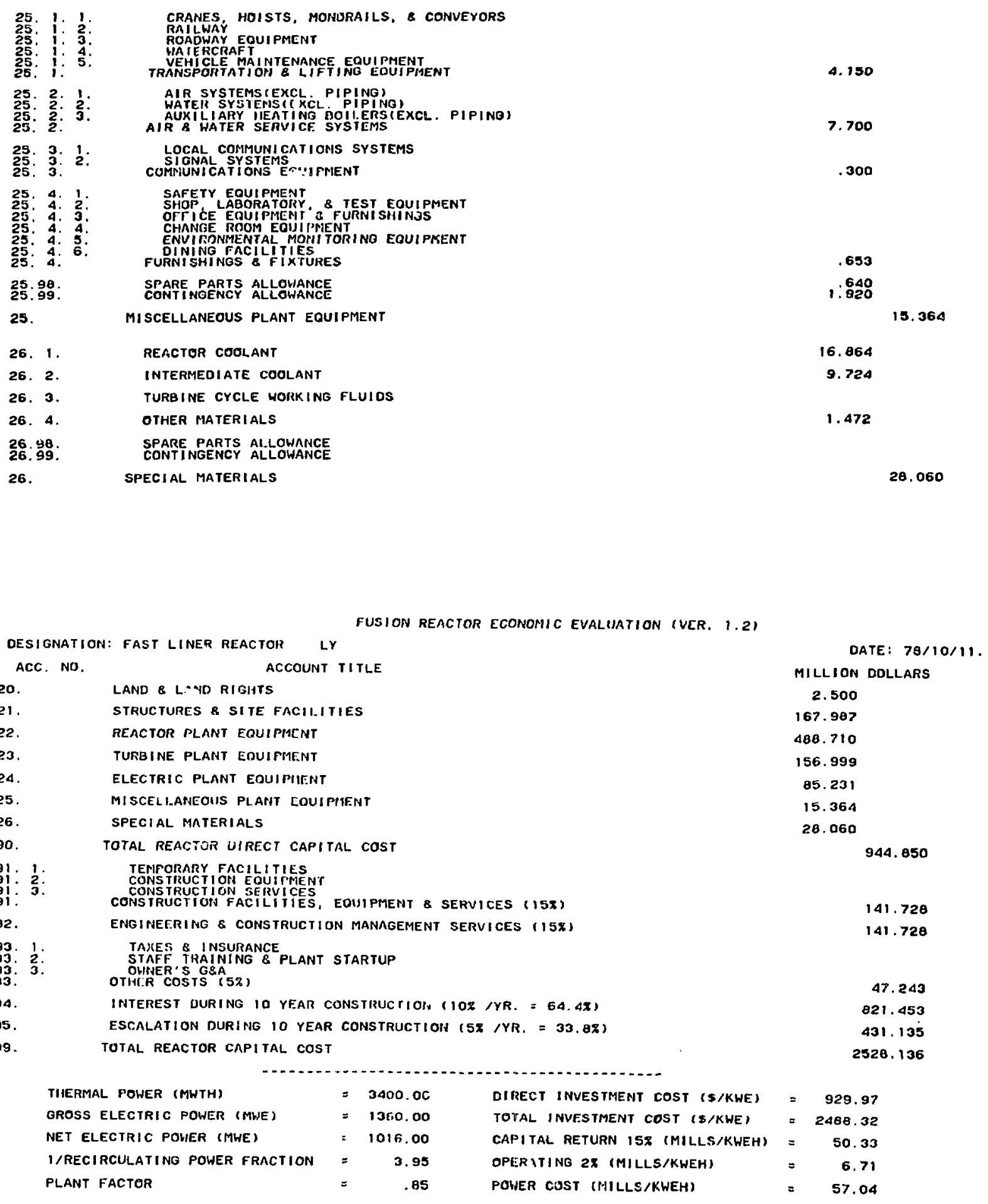




\section{REFERENCES}

1. J. W. Shearer and W. C. Condit, "Magnetically Driven Liner for Plasma Compression," Energy Storage, Compression and Switching (1976) (Plenum Pub7ishing Corporation, NY, 1977), p.705.

2. S. G. Alikhanov, V. P. Bakhtin, W. M. Brusnikin, I. S. Glushkov, R. Kh. Kurtmullaev, and A. L. Lunin, "Studies of Models of Thermonuclear Systems," Proc. 6th International Conf. on Plasma Physics and Controlled Nuc lear Fusion, IAEA-CN-35/14, Berchtesgaden, FRG October 1976.

3. D. L. Book, A. L. Copper, R. Ford, D. Hammer, D. J. Jenkins, A. E. Robson, and P. J. Turchi, "Stabilized Imploding Liner Fusion Systems," Proc. 6th Int. Conf. on Plasma Physics and Controlled Nuclear Fusion Research, Berchtesgaden, FGR, October 1976 (IAEA, Vienna, to be published).

4. C. Rioux and C. Jablon, "Losses of a Thermonuclear Plasma Imploded by a High-Velocity Wal1," Nucl. Fusion 15, 425 (1975).

5. A. R. Sherwood, B. L. Freeman, R. A. Gerwin, T. R. Jarboe, R. A. Krakowski, and R. C. Malone, "Fast Liner Proposal," Los A7amos Scientific Laboratory report LA-6707-P (August 1977).

6. S. G. Alikhanov, V. G. Belan, A. I. Ivanchenko, V. N. Karasjuk, and G. N. Kichigin, "The Production of Pulsed Megagauss Fields by Compression of the Metallic Cylinder in Z-Pinch Configuration," J. Scientific Instruments (J. Phys. E.), Series 2 1, 543 (1963).

7. A. Barcilon, D. L. Book, and A. L. Cooper, "Hydrodynamic Stability of a Rotating Liner," Phys. Fluids 17, 9, 1707 (1974).

8. B. R. Suydam, "Another Look at the Buckling Instability," personal cormunication, Los Alamos Scientific Laboratory (1977).

9. R. A. Krakowski and R. W. Moses, "Energy-Balance and Blast Containment Considerations for FLR," Los Alamos Scientific Laboratory (unpublished data) (1977).

10. R. A. Krakowski, R. W. Moses, R. L. Miller, and R. A. Gerwin, "Fusion Power from Fast Imploding Liners," IAEA Conf. and Workshop on Fusion Reactor Design, Madison, WI, October 10-21, 1977.

11. R. W. Moses, R. A. Krakowski, and R. L. Miller, "Fast-Imp?oding-Liner Fusion Power," Proc. 3rd ANS Meeting on the Technology of Controlleci Nuclear Fusion, Santa Fe, NM, May 0.-11, 1978.

12. R. J. Burke, "Outline for a Large-Pulse Electron-Beam Ignited Fusion Reactor," Los Alamos Scientific Laboratory internal document (1974).

13. T. A. 0liphant and G. E. Gryczkowski, personal communication, Los Alamos Scientific Laboratory (1977).

14. S. I. Braginskii, "Transport Phenomena in a Completely Ionized Two-Temperature Plasma," Sov. Phys. JETP $\underline{6}$ (33), 358-369 (February 1958). 
15. S. Glasstone and R. H. Lovberg, Controlled Thermonuclear Reactions: An Introduction to Theory and Experiment (Van Nostrand Reinhold Co., New York, 1960) p. 37 .

16. T. R. Jarboe, personal communication, Los Alamos Scientific Laboratory (May 1977).

17. R. A. Gerwin and R. C. Malone, "Theory of Plasma Heating by Fast Liner Implosion," submitted to Nucl. Fusion (1977).

18. V. N. Mineev and E. V. Savinov, "Viscosity and Melting Point of Aluminum, Lead, and Sodium Chloride Subjected to Shock Compression," Sov. Phys. JETP 25, 411 (1967).

19. A. R. Sherwood, personal communication, Los Alamos Scientific Laboratory (1978).

20. M. L. Wilkins and M. W. Guinan, "Impact of Cylinders on a Rigid Boundary," J. App1. Phys. 44, 1200 (1973).

21. C. M. Fowler, W. B. Garn, and R. S. Caird, "Production of Very High Magrietic Fields by Implosion," J. Appl. Phys. 31, 588 (1960).

22. C. M. Fowler, R. S. Caird, W. B. Garn, and D. B. Thomson, "The Los Alamos Flux Compression Program from its Origin," R. S. Caird, W. B. Garn, D. B. Thomson, and C. M. Fowler, "A Cylindrical Explosive Flux-Compression System," D. B. Thomson, R. S. Caird, W. B. Garn, and C. M. Fowler, "Plasma Compression by Explosively Produced Magnetic Fields, all in Proceedings of the Conference on Megagauss Magnetic Field Generation by Explosives and Related Experiments, H. Knoepfel and F. Herlack, Eds., Frascati, Italy, September 1965 (Euratom, Brussels, 1966, report EUR 2750.e).

23. R. A. Gerwin, personal communication, Los Alamos Scientific Laboratory (March 1978).

24. R. A. Gerwin and R. W. Moses, "Plasma Heating and Fusion Energy Production by a Z-Pinch Driven Compressible Liner, "Los Alamos Scientific Laboratory, to be published.

25. J. Marshall and I. Hennins, "Fast Plasma from a Coaxial Gun," Plasma Phys. and Controlled Nucl. Fusion Reś. II, 449, IAEA, Vienna (1966).

26. R. A. Gross, Y. G. Chen, E. Halmoy, and R. Moriette, "Strong Shock Waves," Phys. Rev. Lett. 25, 575 (1970).

27. T. R. Jarboe and W. R. Baker, "Apparatus for Producing Laser Targets of 50 Micron Deuterium Pellets," Rev. Sci. Instrum. 45, 431 (1974).

28. G. C. Vlases, personal communication, University of Washington (1978).

29. L. E. Thode, personal communication, LoS Alamos Scientific Laboratory (1978). 
30. R. E. Stillwagon, "Design Study of Reversible Energy Storage and Transfer for the Reference Theta-Pinch Reactor," Westinghouse Electric Corporation Report EM-4620 (September 1974).

31. LASL Group TD-6, "MCNP-A General Monte Carlo Code for Neutron or Photon Transport, Los A7amos Scientific Laboratory report LA-7396-M (JuTy 1978).

32. L. Dresner, "Mechanical Stress in the Pressure Vessel of a Lithium-Filled, Exploding Pellet, Thermonuclear Reactor," Oak Ridge National Laboratory report ORNL-TM-4050 (1973).

33. A. P. Fraas, "The BLASCON - An Exploding Pellet Reactor," Oak Ridge Nationa1 Laboratory report ORNL-TM-3231 (July 1971).

34. I. Q. Bohachevsky, personal communication, Los Alamos Scientific Laboratory (1978).

35. T. R. Neal and R. H. Barnes, personal communication, Los Alamos Scientific Laboratory (1977).

36. R. W. Moses, "Flywheel Energy Storage," Phys. Today 15 (October 1975).

37. C. L. Longmire, Elementary Plasma Physics (Interscience Publishers, NY, 1963).

38. S. Timoshenko and J. N. Goodier, Theory of Elasticity, 2nd Ed. (McGraw-Hi11 Book Co., Inc., 1951).

39. W. Fickett, "PAD, A One-Dimensional Lagrangian Hydrocode," Los Alamos Scientific Laboratory report LA-5910-MS (Apri] 1975).

40. L. F. Coffin, "Fatigue at High Temperature - Predictions and Interpretation," Proc. Inter im. Mech. Engrs. (London) 188, 109 (1974).

41. S. C. Schulte and J. R. Young, "Fusion Reactor Design Studies-Economic Evaluation Guidelines," Baitelle Pacific Northwest Laboratories report PNL-SA-6648 (November 1977).

42. S. C. Schulte and T. L. Willke, "Normalized Fusion Reactor Power Plant Cost Estimtes," 3rd ANS Topical Meeting on the Technology of Controlled Nuclear Fusion, Santa Fe, NM May 9-11, 1978.

43. S. C. Schulte, personal communication, (1978).

44. C. A. Kot, "Shock and Blast Loads in Large Pulse, Inertial Confinement Fusion Reactors," Argonne Nationa1 Laboratory report ANL/CTR/TM-44 (June 1975).

45. N. A. Amherd, "EPRI Alternative Fusion Concepts Evaluation," to be published as EPRI report, 1979).

46. I. R. Lindemuth and T. R. Jarboe, "Initial Numerical Studies of the Behavior of Z-Pinch Plasma Under Liner Implosion Conditions," Nucl. Fusion, 18, 929 (1978). 
47. R. Blewett, personal communication, Glenshaw Glass Co., Glenshaw, PA (1977).

48. M. H. Rice, R. G. McQueen, and J. M. Walsh, "Compression of Solids by Strong Shock Waves," in 6 Solid State Physics (Academic Press, Inc., NY, 1958) pp. 1-60.

49. B. D. Trott, J. E. Backofen, J. J. White, III, "The Design, Evaluation and Delivery of a Prototype, Trailer-Mounted Chamber Capable of Complete Containment of the Blast from Forty Pounds of TNT," Final Report Contract No. N00174-74-C-0218, Battelle Columbus Laboratories, September 1975.

50. D. L. Phung, "A Method for Estimating Escalation and Interest During Construction," personal communication, Oak Ridge National Laboratory (October 1977). 